SUPPORTING INFORMATION

\title{
Structure-Odor Activity Studies on Monoterpenoid Mercaptans Synthesized by Changing the Structural Motifs of the Key Food Odorant 1-p-Menthene-8-thiol
}

Sebastian Schoenauer and Peter Schieberle*

Lehrstuhl für Lebensmittelchemie, Technische Universität München, Lise-MeitnerStraße 34, D-85354 Freising, Germany

Corresponding author e-mail: peter.schieberle@Irz.tum.de Phone: +49 8161712932 ,

Fax: +498161712970 
$\underline{\text { Index }}$

\begin{tabular}{|c|c|}
\hline Hydrogenation of unsaturated monoterpenoids & p. 4 \\
\hline Reduction of ketones and carboxylic acids, respectively, to alcohols & p. 4 \\
\hline $\begin{array}{l}\text { Synthetic route used in the preparation of } 1-p \text {-menthene-8-thiol and its } \\
\text { enantiomers, respectively }\end{array}$ & p. 5 \\
\hline Compounds 1a, 1b, and 1c ( 1-p-menthene-8-thiol and its enantiomers ) & p. 6 \\
\hline Synthetic route $A$ for mercaptans & p. 8 \\
\hline Compound 6a ( $p$-menthane-9-thiol, diastereomer 1) & p. 12 \\
\hline Compound 6b ( $p$-menthane-9-thiol, diastereomer 2) & p. 14 \\
\hline Compound 7 ( (1R,4R)-p-menthane-7-thiol ) & p. 18 \\
\hline Compound 8a ( (1R,2S,4R)-p-menthane-2-thiol ) & p. 21 \\
\hline Compound 8b ( (1S,2R,4S)-p-menthane-2-thiol ) & p. 24 \\
\hline Compound 9a ( (1R,3S,4S)-p-menthane-3-thiol ) & p. 27 \\
\hline Compound 9b ( (1R,3R,4R)-p-menthane-3-thiol ) & p. 29 \\
\hline Compound 9c ( (1S,3R,4R)-p-menthane-3-thiol ) & p. 31 \\
\hline Compound 12 ( $p$-cymene-9-thiol ) & p. 33 \\
\hline Compound 15a ( (1R,2S,4R)-8-p-menthene-2-thiol ) & p. 35 \\
\hline Compound 15b ( (1S,2R,4S)-8-p-menthene-2-thiol ) & p. 37 \\
\hline Compound 16a ( (1R,3S,4S)-8-p-menthene-3-thiol ) & p. 39 \\
\hline Compound 16b ( (1S,3R,4R)-8-p-menthene-3-thiol ) & p. 41 \\
\hline Compound 18a ( (3R)- $\beta$-thio citronellol ) & p. 44 \\
\hline Compound 18b ( (3S)- $\beta$-thio citronellol ) & p. 46 \\
\hline Compound 22 ( dihydro thio citronellol) & p. 48 \\
\hline Synthetic route $B$ for mercaptan preparation & p. 50 \\
\hline Compound 5 ( (1R,4R)-p-menthane-8-thiol ) & p. 51 \\
\hline Compound 10 ( $p$-menthane-4-thiol ) & p. 53 \\
\hline Compound 11 ( $p$-cymene-8-thiol) & p. 55 \\
\hline Compound 13 ( $p$-cymene-7-thiol ) & p. 57 \\
\hline Compound 17 ( (4R)-1,8-p-menthene-3-thiol ) & p. 59 \\
\hline Compound 23 ( tetrahydro thio citronellol ) & p. 61 \\
\hline Compound 24 ( tetrahydro thio linalool ) & p. 63 \\
\hline
\end{tabular}




\begin{tabular}{|l|l|}
\hline $\begin{array}{l}\text { Synthetic route used in the preparation of two diastereomers of 1-p- } \\
\text { menthene-3-thiol (compounds 4a and 4b) from linalool using synthetic route } \\
\text { B }\end{array}$ & p. 65 \\
\hline Compound 4a ( 1-p-menthene-3-thiol, diastereomer 1) & p. 66 \\
\hline Compound 4b ( 1-p-menthene-3-thiol, diastereomer 2) & p. 67 \\
\hline Synthesis of 1-p-menthene-4-thiol (compound 3) & p. 70 \\
\hline Compound 3 ( 1-p-menthene-4-thiol ) & p. 72 \\
\hline Synthesis of thio geraniol (compound 19) and thio nerol (21) & p. 74 \\
\hline Compound 19 ( thio geraniol ) & p. 75 \\
\hline Compound 21 ( thio nerol ) & p. 76 \\
\hline Synthesis of thio linalool (compound 20) & p. 79 \\
\hline Compound 20 ( thio linalool) & p. 80 \\
\hline Further spectroscopic data & p. 82 \\
\hline Compound 14 ( p-cymene-3-thiol) & p. 82 \\
\hline
\end{tabular}




\section{Syntheses and substance characterization}

2

3 If the respective alcohols for the $\mathrm{OH} / \mathrm{SH}$-exchange reaction were commercially not 4 available, these were either obtained by hydrogenation of unsaturated analogues or

\section{Hydrogenation of unsaturated monoterpenoids}

The respective monoterpenoid $(10 \mathrm{mmol})$ was dissolved in methanol $(50 \mathrm{~mL})$ and platinum(IV) oxide (a spatula tip full) was added. Hydrogenation was performed in an autoclave by means of hydrogen for $2 \mathrm{~h}$ at a pressure of 5 bar. The solution was filtered and the solvent was removed under reduced pressure.

\section{Reduction of ketones and carboxylic acids}

Carboxylic acids or ketones $(10 \mathrm{mmol})$ were dissolved in anhydrous diethyl ether $(30 \mathrm{~mL})$ and cooled to $0^{\circ} \mathrm{C}$. A solution of lithium aluminium hydride $(0.78 \mathrm{~g} ; 20 \mathrm{mmol})$ in anhydrous diethyl ether $(30 \mathrm{~mL})$ was slowly added and then stirred at RT for $2 \mathrm{~h}$. Anhydrous diethyl ether $(50 \mathrm{~mL})$, saturated ammonium chloride solution $(30 \mathrm{~mL})$, and $\mathrm{HCl}(2 \mathrm{~mol} / \mathrm{L} ; 30 \mathrm{~mL})$ were carefully added at $0{ }^{\circ} \mathrm{C}$. The aqueous layer was washed twice with diethyl ether $(50 \mathrm{~mL})$, the combined organic layers were washed twice with saturated sodium hydrogen carbonate solution $(30 \mathrm{~mL})$ and finally dried over sodium 3 sulfate. The solvent was evaporated in vacuo and the residue was purified by column chromatography on silica gel using pentane as the eluent. 


\section{Synthetic route used in the preparation of 1-p-menthene-8-thiol and its} enantiomers, respectively

1-p-Menthene-8-thiol and its enantiomers were synthesized using Lawesson's reagent (Figure S1).

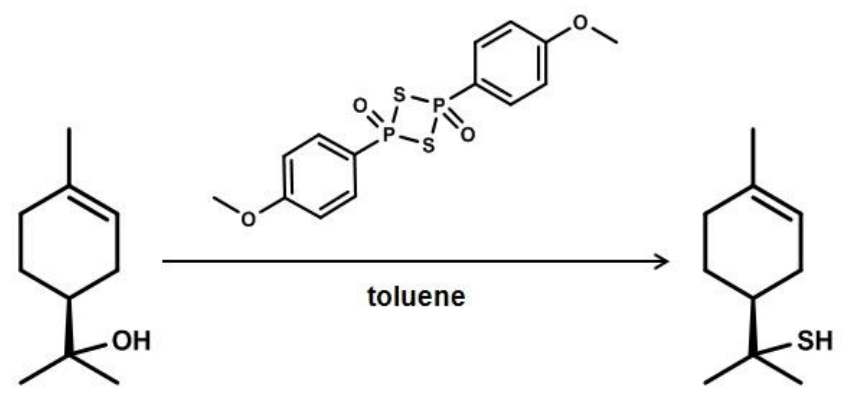

Figure S1. Synthetic route used for the enantioselective preparation of 1-pmenthene-8-thiol.

Lawesson's reagent $(4.05 \mathrm{~g} ; 10 \mathrm{mmol})$ was dissolved in toluene $(150 \mathrm{~mL})$. Then racemic $\alpha$-terpineol or the $(R)$ - and $(S)$-enantiomer $(10 \mathrm{mmol})$ was added, and the mixture was stirred for $2 \mathrm{~h}$ at $120^{\circ} \mathrm{C}$. After cooling, the solvent was removed under reduced pressure and the residue was dissolved in dichloromethane. The remaining salt was removed by SAFE-distillation. The distillate was dried over anhydrous sodium sulfate and after removal of the solvent, the product was purified by means of column chromatography on silica gel (silica 60, 0.040 - 0.063 mm) (Merck, Darmstadt, Germany) using pentane as the eluent. 
47

48

49

50

51

52

53

54

55

56

57

58

59

60

61

62

63

64

65

66

67

68

Compounds $\mathbf{1 a}, \mathbf{1 b}$ and $\mathbf{1 c}$

1-p-menthene-8-thiol, yield: $4 \%$

(R)-1-p-menthene-8-thiol, yield: $6 \%$

(S)-1-p-menthene-8-thiol, yield: $3 \%$

educts: $\alpha$-terpineol, $(R)-(+)$ - $\alpha$-terpineol, $(S)-(-)$ - $\alpha$-terpineol

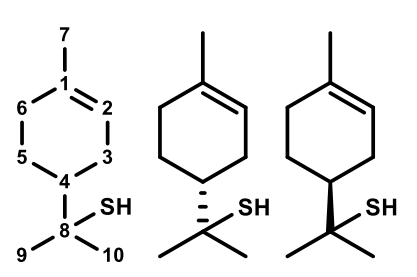

RI (FFAP): 1577

RI (DB-5): 1285

MS-El: $m / z$ (intensity in \%) 121 (100), 136 (82), 93 (80), 41 (35), 75 (35), 81 (33), 67

(25), 79 (25), 95 (25), 69 (22), 77 (22), 91 (21), 53 (15), 68 (15), 55 (13), 92 (12), 107 (12), 137 (12), 43 (11), 94 (11), $170\left(10, M^{+}\right), 65$ (8), 105 (8), 122 (8), 59 (7), 108 (6), $155(5)$

MS-Cl (isobutane): m/z (intensity in \%) 137 (100), 136 (18), 138 (12), 171 (12, $\left.[\mathrm{M}+\mathrm{H}]^{+}\right), 93(7), 95(5)$

${ }^{1} \mathrm{H}$ NMR (400 MHz; $\mathrm{CDCl}_{3}$ ): $\delta 1.36$ (s, 3H, H-C9), $1.33-1.37$ (m, 1H, H-C5), 1.42 (s, $3 \mathrm{H}, \mathrm{H}-\mathrm{C} 10), 1.45$ (s, SH), $1.54-1.59$ (m, 1H, H-C4), 1.67 (s, 3H, H-C7), 1.91 - 1.93 (m, 1H, H-C3), 1.96 - 1.97 (m, 1H, H-C5), 1.99 - 2.03 (m, 2H, H-C6), 2.11 - 2.17 (m, $1 \mathrm{H}, \mathrm{H}-\mathrm{C} 3), 5.38-5.41(\mathrm{~m}, 1 \mathrm{H}, \mathrm{H}-\mathrm{C} 2)$

${ }^{13} \mathrm{C}$ NMR (101 MHz, CDCl 3 ): $\delta 23.27$ (C7), 24.86 (C5), 27.39 (C3), 30.17 (C9), 31.14 (C6), 31.46 (C10), 45.95 (C4), 47.91 (C8), 120.48 (C2), 133.88 (C1) 


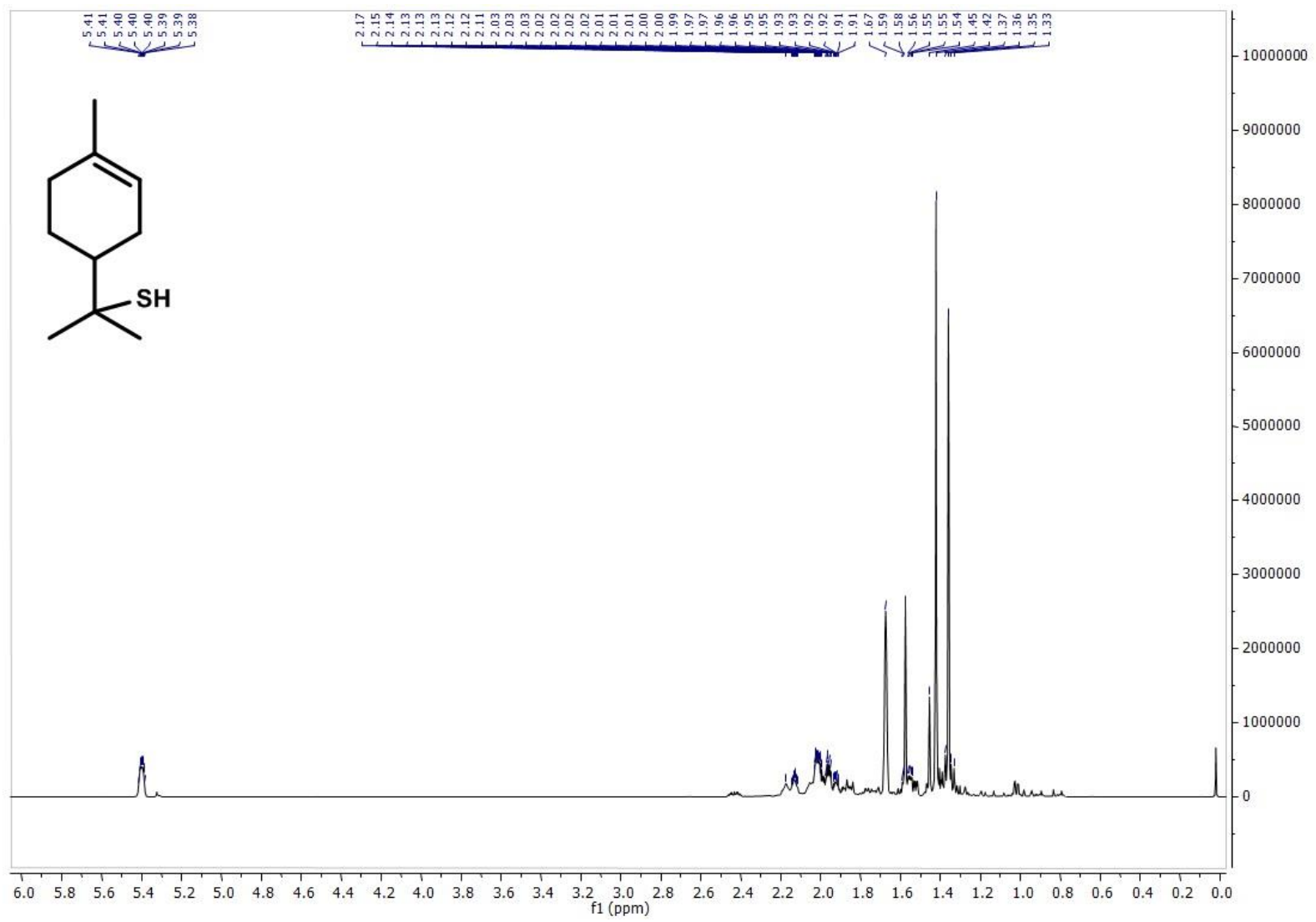

Figure S2. ${ }^{1} \mathrm{H}$ NMR spectrum of 1-p-menthene-8-thiol.

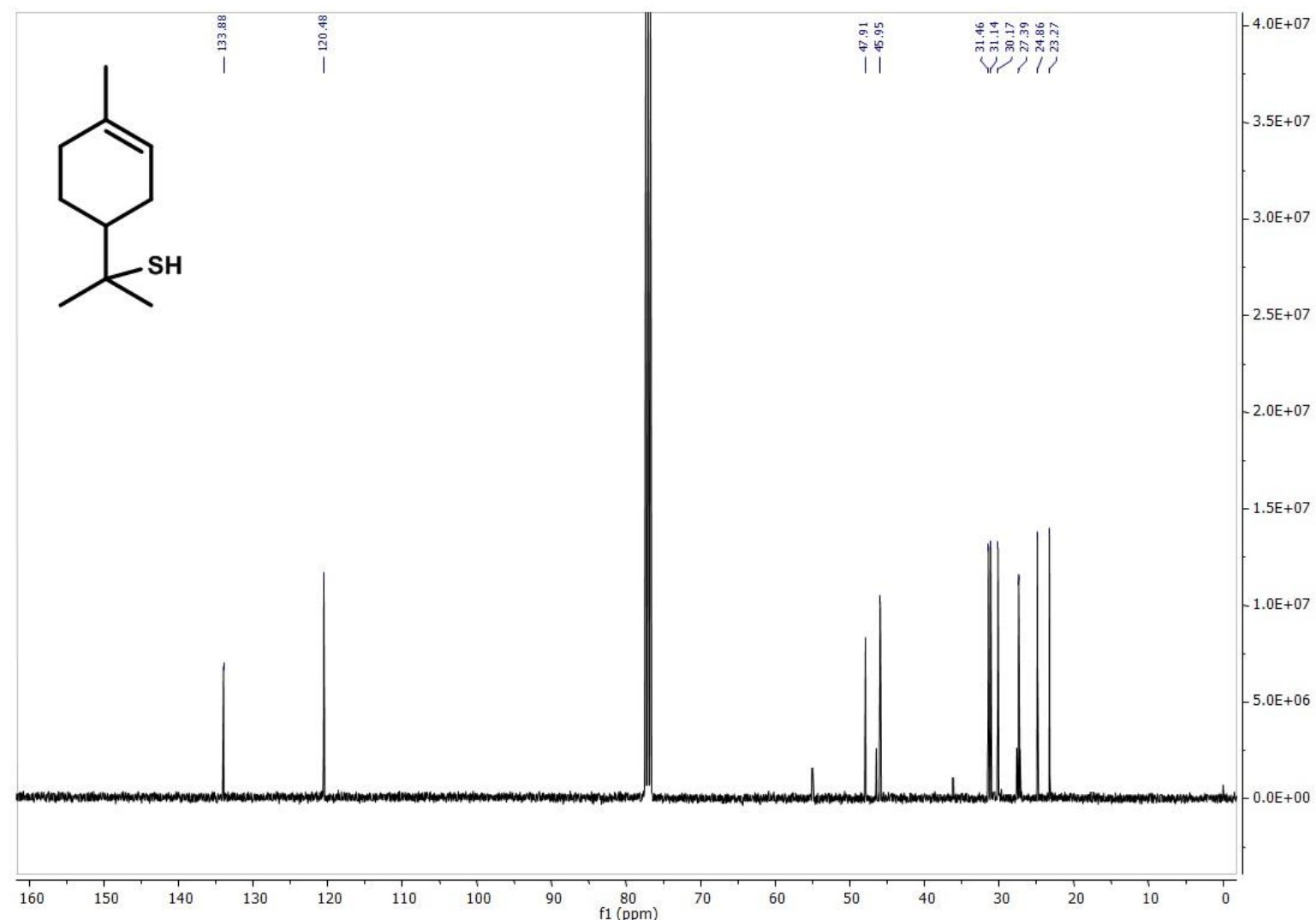

73 Figure S3. ${ }^{13} \mathrm{C}$ NMR spectrum of $1-p$-menthene-8-thiol. 


\section{Synthetic route A for mercaptan preparation}

Seventeen compounds were obtained by using the strategy consisting of tosylation, thioacetylation, and reduction as shown in Figure S4.

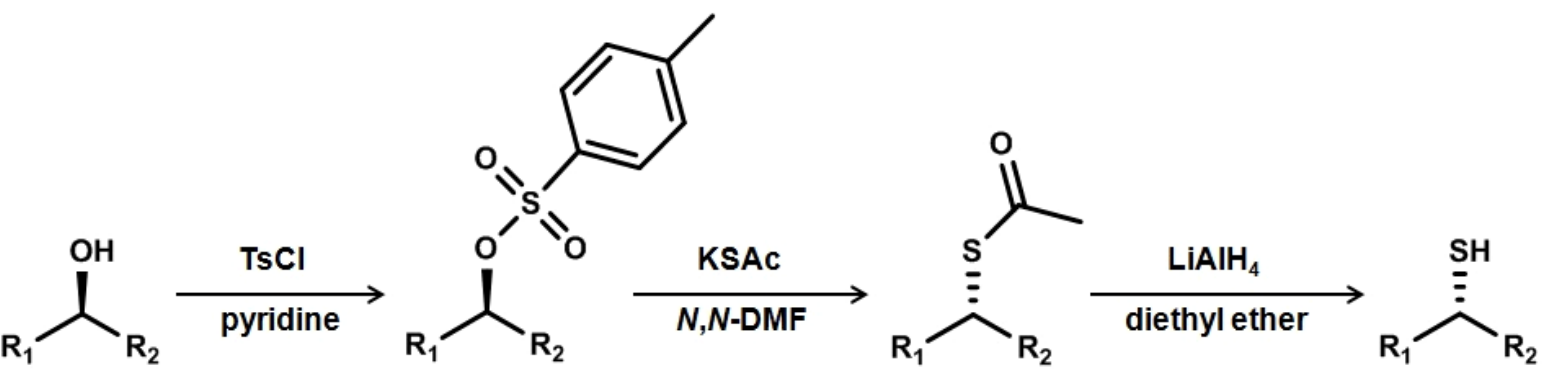

$$
R_{1}, R_{2}=H, \text { alkyl }
$$

Figure S4. Route assigned as "synthetic route A".

a) Tosylation. The respective alcohol $(10 \mathrm{mmol})$ was dissolved in pyridine $(20 \mathrm{~mL})$ and cooled to $0^{\circ} \mathrm{C}$. $p$-Toluene sulfonyl chloride $(2.10 \mathrm{~g} ; 11 \mathrm{mmol})$ was added, and the solution was stirred overnight at RT. Hexane (30 mL) was added, the reaction mixture was filtered, and the residue was washed with hexane $(30 \mathrm{~mL})$. The organic layer was carefully extracted with $\mathrm{HCl}(5 \mathrm{~mol} / \mathrm{L} ; 30 \mathrm{~mL})$ and dried over sodium sulfate. The solvent was removed under reduced pressure.

b) Thioacetylation. The tosylate $(7 \mathrm{mmol})$ was dissolved in $\mathrm{N}, \mathrm{N}$ dimethylformamide $(20 \mathrm{~mL})$ and potassium thioacetate $(2.39 \mathrm{~g} ; 21 \mathrm{mmol})$ was added. The solution was stirred at $85^{\circ} \mathrm{C}$ for $2 \mathrm{~h}$ and then diluted with diethyl ether $(30 \mathrm{~mL})$. The organic layer was washed twice with brine $(20 \mathrm{~mL})$, dried over sodium sulfate and concentrated under reduced pressure. The thioacetate was purified by means of column chromatography on silica gel using pentane/diethyl ether ( $98+2$; by vol.) as the eluent.

c) Reduction. The thioacetate $(4 \mathrm{mmol})$ was dissolved in anhydrous diethyl ether $(15 \mathrm{~mL})$ and cooled to $0{ }^{\circ} \mathrm{C}$. A solution of lithium aluminium hydride $(0.31 \mathrm{~g} ; 8 \mathrm{mmol})$ in anhydrous diethyl ether $(15 \mathrm{~mL})$ was slowly added and then stirred at RT for $2 \mathrm{~h}$. Anhydrous diethyl ether $(30 \mathrm{~mL})$, saturated ammonium chloride solution $(20 \mathrm{~mL})$, and 
$103 \mathrm{HCl}(2 \mathrm{~mol} / \mathrm{L} ; 20 \mathrm{~mL})$ were added at $0{ }^{\circ} \mathrm{C}$. The aqueous layer was washed twice with 104 diethyl ether $(30 \mathrm{~mL})$, the combined organic layers were washed twice with saturated 105 sodium hydrogen carbonate solution $(20 \mathrm{~mL})$ and dried over sodium sulfate. The 106 solvent was evaporated in vacuo and the residue was purified by column 107 chromatography on silca gel using pentane as the eluent. 
109

110

111

112

113

114

115

116

117

118

119

120

121

122

123

124

125

126

127

128

129

130

131

132

133

134

135

136

137

138

139

140

141

142

Compound 2

\section{$(4 S, 8 R)-1-p$-menthene-9-thiol}

educt: $(4 S, 8 R)-1-p$-menthene-9-ol

a) $1^{\text {st }}$ intermediate:

(R)-2-((S)-4-methylcyclohex-3-en-1-yl)propyl 4-methylbenzenesulfonate, yield: $73 \%$

b) $2^{\text {nd }}$ intermediate:

S-((R)-2-((S)-4-methylcyclohex-3-en-1-yl)propyl) ethanethioate, yield: $84 \%$,

MS-El: m/z (intensity in \%) 169 (100), 43 (52), 93 (38), 95 (36), 79 (25), 81 (24), 135 (24), 94 (20), 41 (18), 67 (18), 55 (16), 107 (15), 121 (15), 170 (15), 77 (12), 75 (10), $91(10), 136(8), 113(6), 53(5), 171(5), 212\left(3, \mathrm{M}^{+}\right)$

c) analyte:

yield: $83 \%$

RI (FFAP): 1710

RI (DB-5): 1364

MS-El: m/z (intensity in \%) 94 (100), 95 (94), 93 (80), 79 (77), 67 (65), 81 (63), 41 (60), $170\left(59, \mathrm{M}^{+}\right), 68$ (53), 91 (44), 55 (43), 136 (43), 77 (40), 121 (32), 107 (30), 53 (28), 123 (28), 75 (26), 92 (22), 69 (18), 113 (18), 168 (18), 47 (16), 65 (15), 80 (15), 137 (15), 168 (15), 153 (14), 78 (11), 96 (10), 105 (10), 108 (10), 155 (10), 171 (9) MS-Cl (isobutane): $m / z$ (intensity in \%) $171\left(100,[\mathrm{M}+\mathrm{H}]^{+}\right), 137$ (34), 93 (14), 95 (12), 123 (12) 170 (11), 91 (7), 172 (5)

${ }^{1} \mathrm{H}$ NMR $\left(400 \mathrm{MHz} ; \mathrm{CDCl}_{3}\right): \delta 0.98(\mathrm{~d}, J=6.6 \mathrm{~Hz}, 3 \mathrm{H}, \mathrm{H}-\mathrm{C} 10), 1.25-1.34(\mathrm{~m}, 1 \mathrm{H}, \mathrm{H}-$ C5), 1.28 (t, J=8.0 Hz, SH), 1.51 - 1.64 (m, 2H, H-C4, H-C8), 1.66 (s, 3H, H-C7), 1.68 - 1.80 (m, 2H, H-C3, H-C5), 1.91 - 2.06 (m, 3H, H-C3, H-C6), 2.42 - 2.49 (m, $1 \mathrm{H}, \mathrm{H}-\mathrm{C} 9), 2.62$ - 2.69 (m, 1H, H-C9), 5.37 - 5.41 (m, 1H, H-C2)

${ }^{13} \mathrm{C}$ NMR (101 MHz, $\mathrm{CDCl}_{3}$ ): $\delta 15.10$ (C10), 23.44 (C7), 27.12 (C5), 27.45 (C3), 29.52 (C9), 30.69 (C6), 36.75 (C4), 40.63 (C8), 120.54 (C2), 134.04 (C1) 


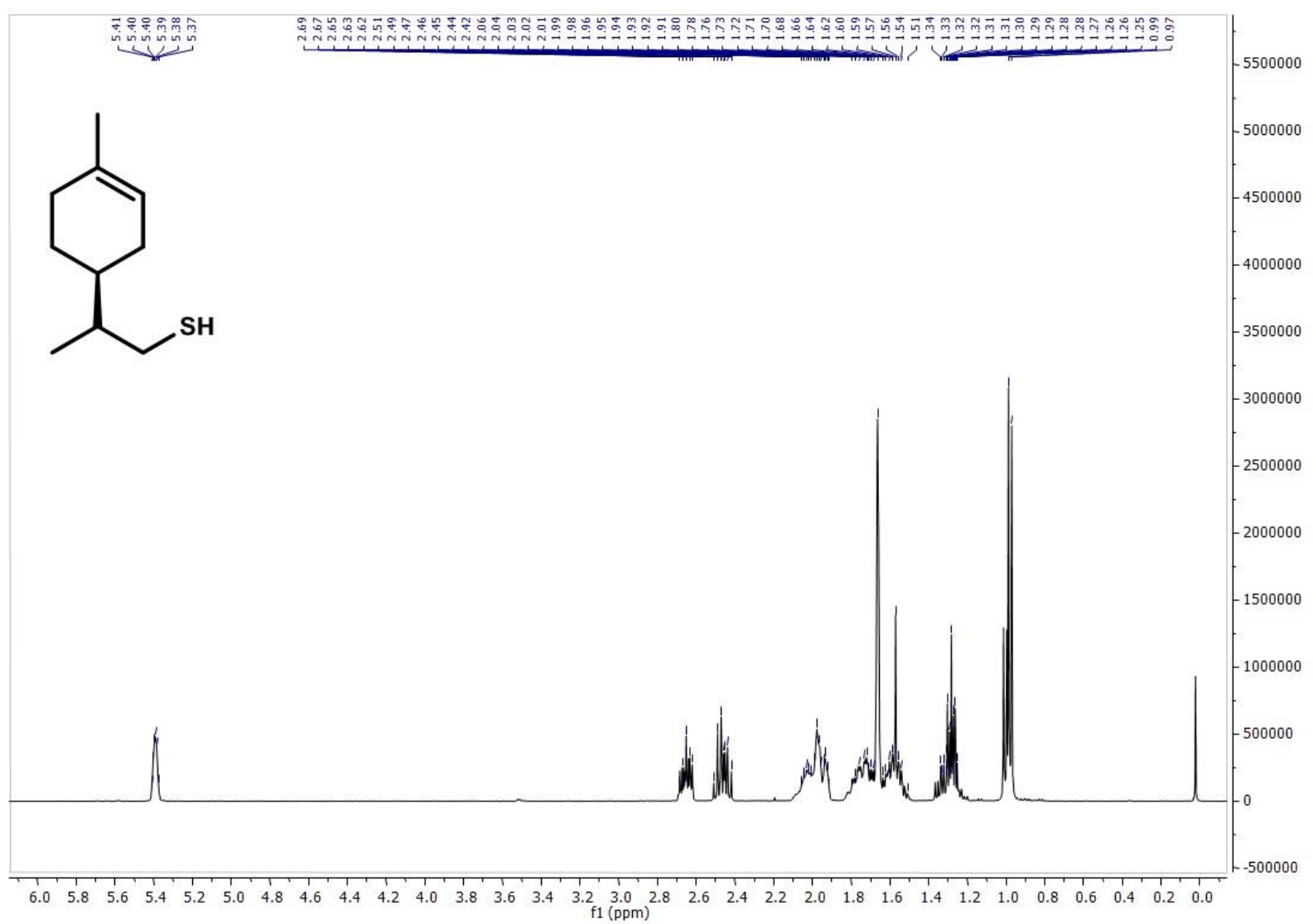

Figure S5. ${ }^{1} \mathrm{H}$ NMR spectrum of $(4 S, 8 R)-1-p$-menthene-9-thiol.

145

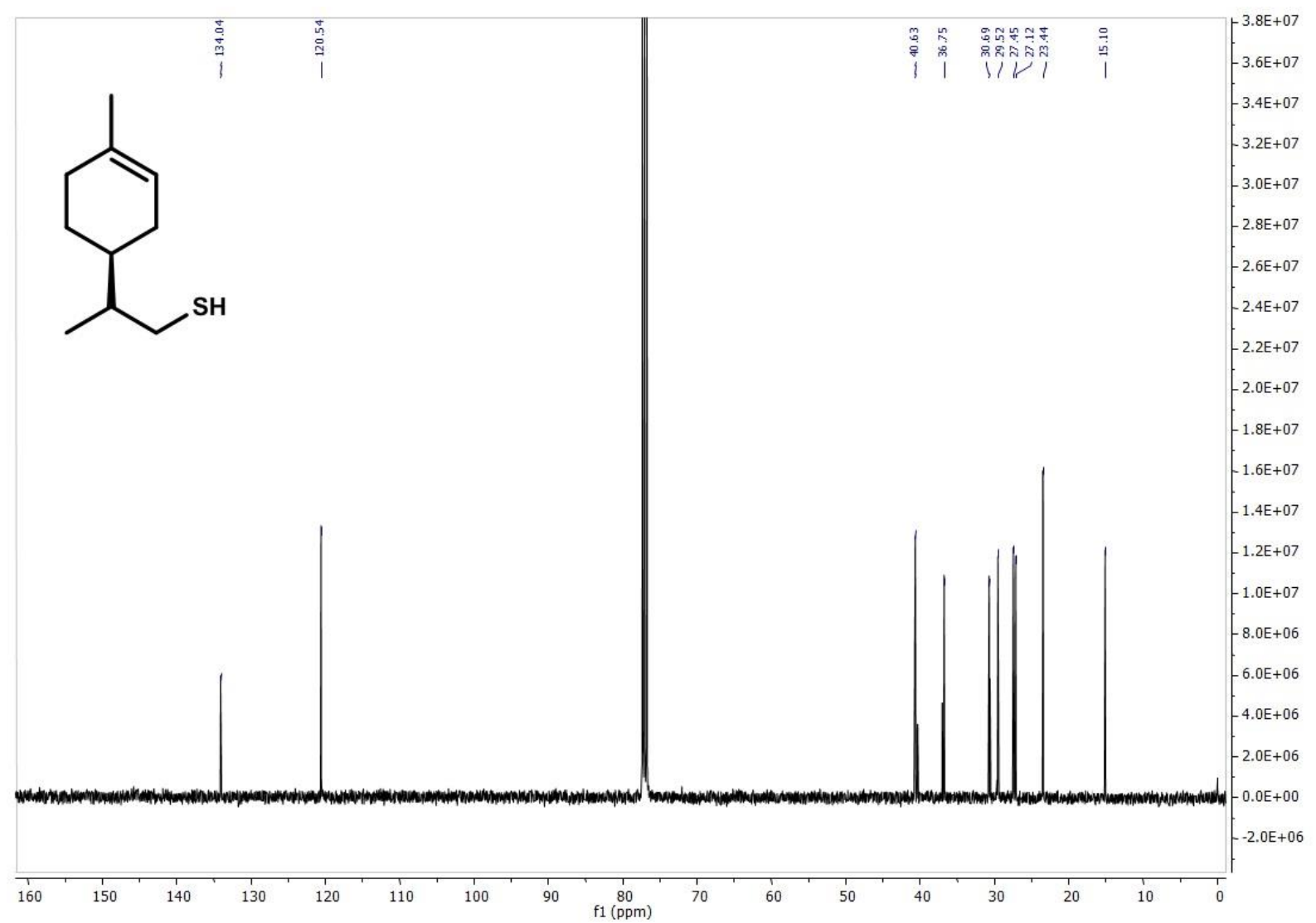

147 Figure S6. ${ }^{13} \mathrm{C}$ NMR spectrum of $(4 S, 8 R)-1$ - $p$-menthene-9-thiol. 
148 Compound 6a

149

150

151

p-menthane-9-thiol, diastereomer 1:

(R)-2-((1s,4S)-4-methylcyclohexyl)propane-1-thiol

152

153

educt: $(4 S, 8 R)-p$-menthene-9-ol

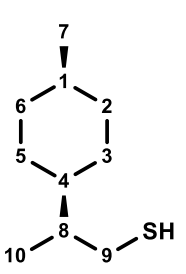

154

155

a) $1^{\text {st }}$ intermediate (obtained by hydrogenation):

156

p-menthane-9-ol, diastereomer 1:

157

(R)-2-((1s,4S)-4-methylcyclohexyl)propane-1-thiol, yield: $50 \%$,

MS-El: m/z (intensity in \%) 55 (100), 69 (67), 81 (51), 96 (45), 95 (38), 41 (29), 97

159

(27), 83 (24), 67 (18), 68 (12), 138 (12), 82 (11), 123 (11), 109 (9), 125 (9), 57 (8), 43

160

(6), 42 (5), 53 (5), 54 (5), 56 (5), 58 (5), M+ absent

161

162

b) $2^{\text {nd }}$ intermediate:

163

(R)-2-((1s,4S)-4-methylcyclohexyl)propyl 4-methylbenzenesulfonate,

164

crude yield: $65 \%$ (sum of diastereomers, see also compound $6 \mathrm{~b}$ )

165

166

c) $3^{\text {rd }}$ intermediate:

167

S-((R)-2-((1s,4S)-4-methylcyclohexyl)propyl) ethanethioate,

168

yield: $45 \%$,

169

MS-El: m/z (intensity in \%) 43 (100), 138 (79), 55 (57), 81 (54), 95 (53), 139 (50), 171

170

(50), 140 (42), 41 (35), 137 (31), 69 (30), 96 (25), 83 (20), 67 (18), 163 (17), 123 (15),

171

82 (13), 180 (13), 97 (11), 79 (10), 109 (10), 77 (9), 170 (8), 42 (7), 53 (7), 165 (7),

172

169 (7), 54 (6), 68 (6), 162 (6), 172 (6), 214 (6, M+), 47 (5), 57 (5), 91 (5), 181 (5),

173

196 (5) 


\begin{tabular}{|c|c|}
\hline 174 & d) analyte: \\
\hline 175 & yield: $36 \%$ \\
\hline 76 & RI (FFAP): 1548 \\
\hline 177 & R (FFAP): 1307 \\
\hline 78 & \\
\hline 79 & MS-El: m/z (intensity in \%) 55 (100), 138 (61), 69 (57), 96 (52), 81 (51), 41 (50), 95 \\
\hline 80 & $(36), 172\left(35, \mathrm{M}^{+}\right), 47(24), 138(23), 82(22), 75(20), 67(19), 83(19), 109(17), 43$ \\
\hline 31 & $(16), 53(15), 79(14), 97(11), 94(10), 139(10), 57(9), 68(9), 77$ (9), $123(8), 74(7)$, \\
\hline 82 & $84(7), 93(7), 54(6), 68(6), 173(6), 171(5)$ \\
\hline 33 & MS-Cl (isobutane): $m / z$ (intensity in \%) $139(100), 173\left(35,[\mathrm{M}+\mathrm{H}]^{+}\right), 128(32), 97$ \\
\hline 184 & $(15), 140(8), 171(8), 174(6)$ \\
\hline 185 & ${ }^{1} \mathrm{H}$ NMR $\left(400 \mathrm{MHz} ; \mathrm{CDCl}_{3}\right): \delta 0.87(\mathrm{~d}, J=6.5 \mathrm{~Hz}, 3 \mathrm{H}, \mathrm{H}-\mathrm{C} 7), 0.88-0.96(\mathrm{~m}, 2 \mathrm{H}, \mathrm{H}-$ \\
\hline 1 & C3, H-C5), $0.94(\mathrm{~d}, \mathrm{~J}=6.8 \mathrm{~Hz}, 3 \mathrm{H}, \mathrm{H}-\mathrm{C} 10), 0.92-1.10(\mathrm{~m}, 2 \mathrm{H}, \mathrm{H}-\mathrm{C} 2, \mathrm{H}-\mathrm{C} 6), 1.24$ (t, \\
\hline 187 & $J=8.0 \mathrm{~Hz}, \mathrm{SH}), 1.23-1.31(\mathrm{~m}, 1 \mathrm{H}, \mathrm{H}-\mathrm{C} 1), 1.26-1.33(\mathrm{~m}, 1 \mathrm{H}, \mathrm{H}-\mathrm{C} 8), 1.42-1.49$ \\
\hline 188 & $(\mathrm{~m}, 1 \mathrm{H}, \mathrm{H}-\mathrm{C} 4), 1.57-1.66(\mathrm{~m}, 1 \mathrm{H}, \mathrm{H}-\mathrm{C} 2), 1.58-1.65(\mathrm{~m}, 1 \mathrm{H}, \mathrm{H}-\mathrm{C} 6), 1.66-1.75(\mathrm{~m}$, \\
\hline T & $2 \mathrm{H}, \mathrm{H}-\mathrm{C} 3, \mathrm{H}-\mathrm{C} 5), 2.37-2.43(\mathrm{~m}, 1 \mathrm{H}, \mathrm{H}-\mathrm{C} 9), 2.57-2.63(\mathrm{~m}, 1 \mathrm{H}, \mathrm{H}-\mathrm{C} 9)$ \\
\hline & ${ }^{13} \mathrm{C}$ NMR (101 MHz, $\left.\mathrm{CDCl}_{3}\right): \delta 15.41$ (C10), 22.66 (C7), 28.40 (C6), 29.57 (C9), \\
\hline & 30.66 (C2), $32.85(\mathrm{C} 1), 35.32(\mathrm{C} 5), 35.44(\mathrm{C} 3), 40.79(\mathrm{C} 8), 41.34$ (C4) \\
\hline
\end{tabular}


192

Compound 6b

193

194

195

196

197

198

199

200

201

202

203

204

205

206

207

208

209

210

211

212

213

214

215

216 yield: $30 \%$, p-menthane-9-thiol, diastereomer 2:

(R)-2-((1r,4R)-4-methylcyclohexyl)propane-1-thiol

educt: $(4 S, 8 R)-p$-menthene-9-ol

a) $1^{\text {st }}$ intermediate (obtained by hydrogenation):

p-menthane-9-ol, diastereomer 2:

(R)-2-((1r,4R)-4-methylcyclohexyl)propane-1-thiol, yield: $35 \%$,

MS-El: m/z (intensity in \%) 55 (100), 69 (51), 81 (51), 96 (42), 95 (37), 41 (30), 97

(24), 83 (19), 67 (17), 68 (13), 82 (12), 123 (11), 138 (10), 109 (9), 43 (7), 57 (7), 42

(6), 53 (6), 54 (6), 56 (6), 58 (6), $125(6), M^{+}$absent

b) $2^{\text {nd }}$ intermediate:

(R)-2-((1r,4R)-4-methylcyclohexyl)propyl 4-methylbenzenesulfonate,

crude yield: $65 \% \%$ (sum of diastereomers, see also compound 6a)

c) $3^{\text {rd }}$ intermediate:

S-((R)-2-((1r,4R)-4-methylcyclohexyl)propyl) ethanethioate,

MS-El: m/z (intensity in \%) 43 (100), 138 (69), 81 (54), 95 (52), 55 (50), 171 (46), 140 (43), 139 (40), 41 (37), 69 (28), 83 (22), 96 (22), 137 (21), 67 (17), 123 (16), $82(14)$, 97 (14), 42 (9), 53 (9), 75 (9), 68 (8), 79 (8), 109 (8), 162 (7), 163 (7), 170 (7), 196 (7), 54 (6), 77 (6), 93 (6), 94 (6), $110(5), 214\left(5, \mathrm{M}^{+}\right)$ 


\begin{tabular}{|c|c|}
\hline 217 & d) analyte: \\
\hline 18 & yield: $23 \%$ \\
\hline 19 & RI (FFAP): 1577 \\
\hline 20 & $R(F F A P): 1324$ \\
\hline & \\
\hline 22 & MS-EI: m/z (intensity in \%) 138 (100), 55 (76), 69 (57), 96 (56), 81 (53), 95 (49), 41 \\
\hline 23 & $(41), 67(32), 47(30), 172\left(30, M^{+}\right), 83(24), 68(18), 57(17), 123(17), 79(15), 82$ \\
\hline 24 & $(15), 53(14), 109(14), 54(13), 139$ (12), 53 (11), 52 (11), 74 (10), 97 (9), 93 (6), 110 \\
\hline 29 & $(6)$ \\
\hline & MS-Cl (isobutane): $m / z$ (intensity in \%) $139(100), 173\left(62,[M+H]^{+}\right), 138(34), 97$ \\
\hline 27 & $(17), 140(9), 171(9), 174(8)$ \\
\hline & ${ }^{1} \mathrm{H}$ NMR $\left(400 \mathrm{MHz} ; \mathrm{CDCl}_{3}\right): \delta 0.85-0.90(\mathrm{~m}, 1 \mathrm{H}, \mathrm{H}-\mathrm{C} 6), 0.91(\mathrm{~d}, J=7.0 \mathrm{~Hz}, 3 \mathrm{H}, \mathrm{H}-$ \\
\hline & C7), $0.96(\mathrm{~d}, J=6.8 \mathrm{~Hz}, 3 \mathrm{H}, \mathrm{H}-\mathrm{C} 10), 1.23(\mathrm{t}, J=8.0 \mathrm{~Hz}, \mathrm{SH}), 1.30-1.38(\mathrm{~m}, 2 \mathrm{H}, \mathrm{H}-$ \\
\hline & C3, H-C5), $1.35-1.42(\mathrm{~m}, 5 \mathrm{H}, \mathrm{H}-\mathrm{C} 2, \mathrm{H}-\mathrm{C} 4, \mathrm{H}-\mathrm{C} 6), 1.44-1.53$ (m, 2H, H-C3, H-C5), \\
\hline & $1.56-1.62(\mathrm{~m}, 1 \mathrm{H}, \mathrm{H}-\mathrm{C} 8), 1.71-1.79(\mathrm{~m}, 1 \mathrm{H}, \mathrm{H}-\mathrm{C} 1), 2.37-2.46(\mathrm{~m}, 1 \mathrm{H}, \mathrm{H}-\mathrm{C} 10)$ \\
\hline & $2.60-2.68(\mathrm{~m}, 1 \mathrm{H}, \mathrm{H}-\mathrm{C} 10)$ \\
\hline & ${ }^{13} \mathrm{C} \mathrm{NMR}\left(101 \mathrm{MHz}, \mathrm{CDCl}_{3}\right): \delta 15.71$ (C10), 19.09 (C7), 24.26 (C6), 25.69 (C2), \\
\hline & 28.91 (C1), 29.65 (C9), 31.29 (C3), 31.40 (C5), 38.69 (C8), 39.79 (C4) \\
\hline
\end{tabular}




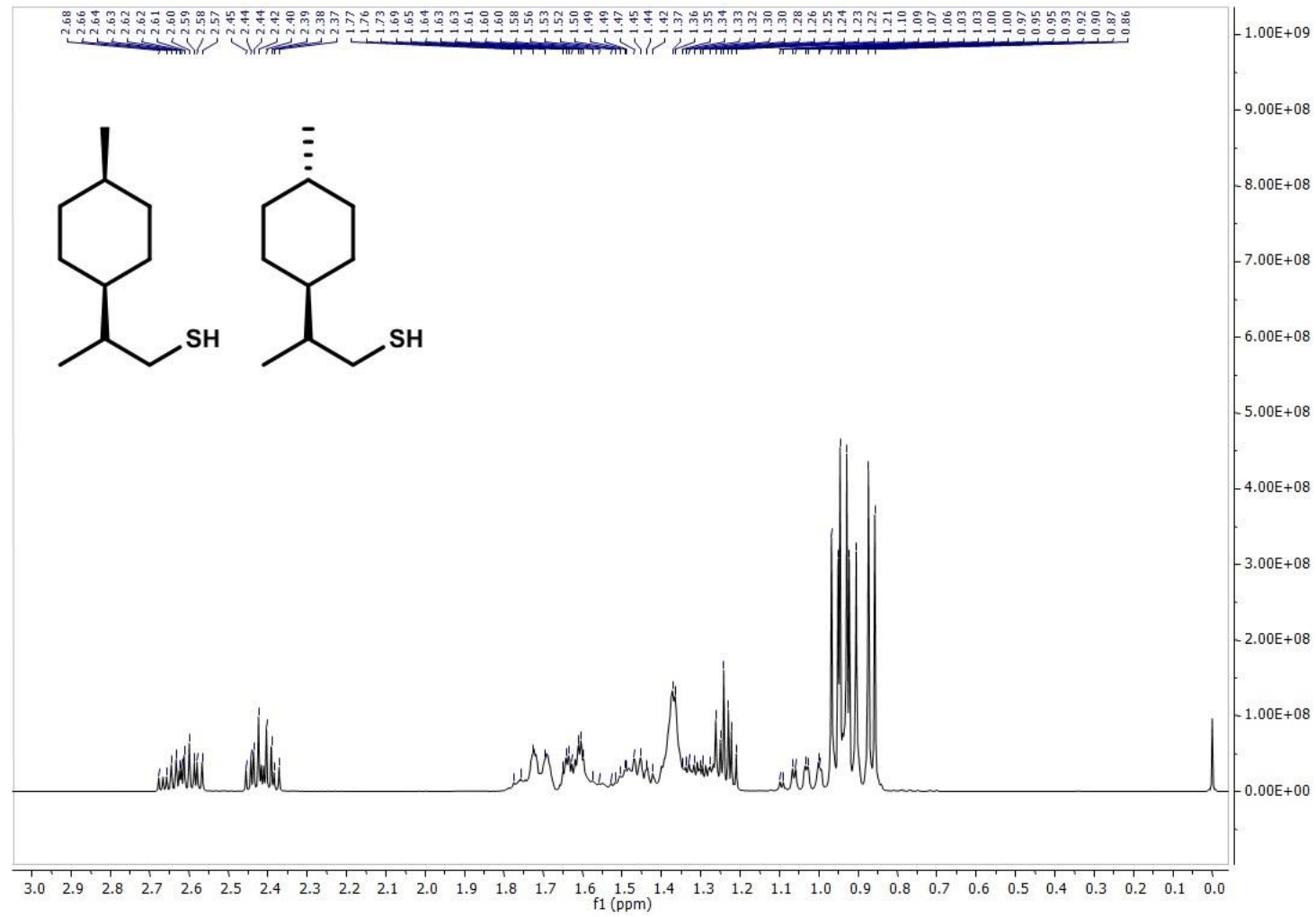

Figure S7. ${ }^{1} \mathrm{H}$ NMR spectrum of $p$-menthane-9-thiol (mixture of 2 diastereomers).

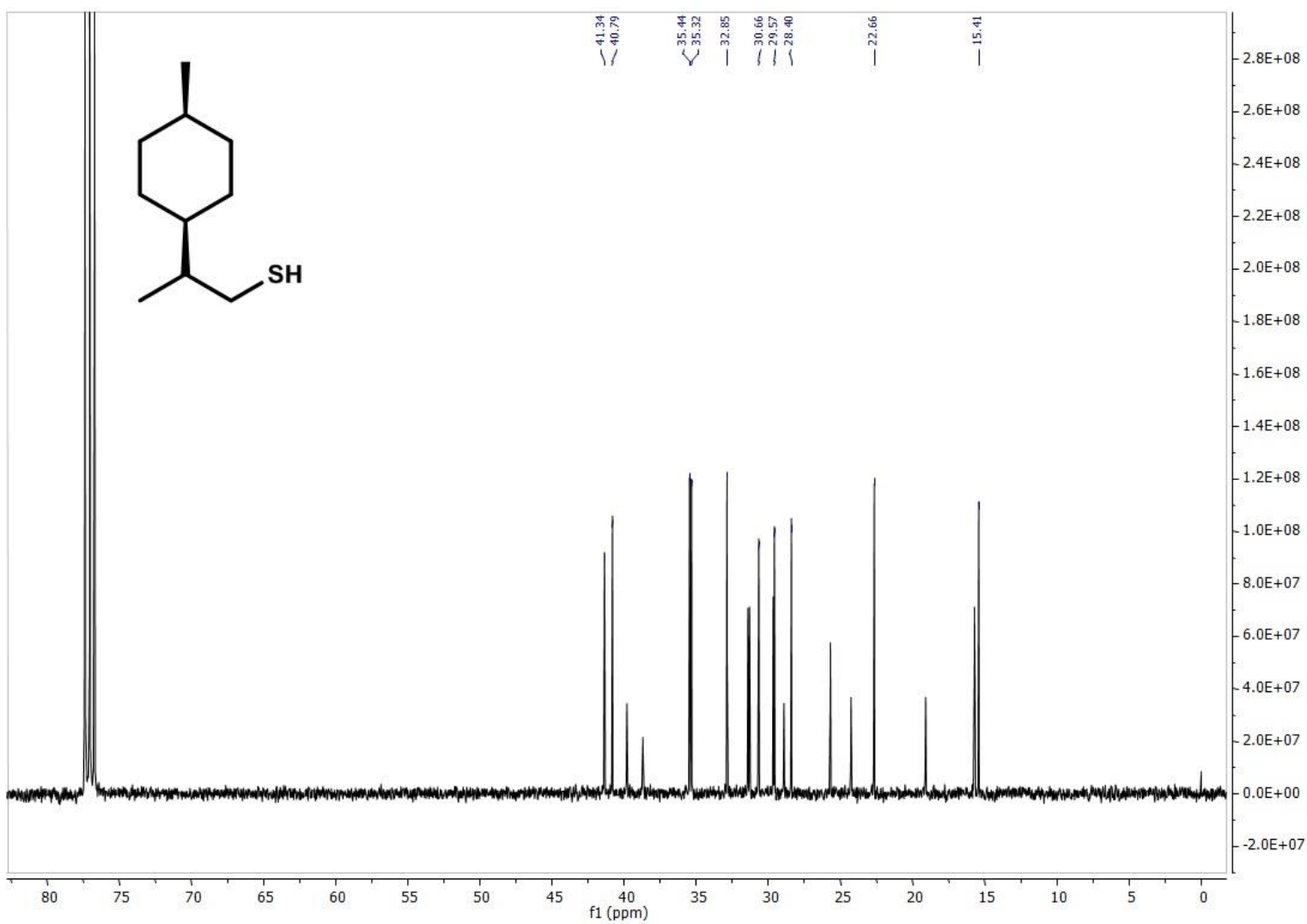

239 Figure S8. ${ }^{13} \mathrm{C}$ NMR spectrum of $p$-menthane-9-thiol (mixture of 2 diastereomers);

240 signals of diastereomer 1. 


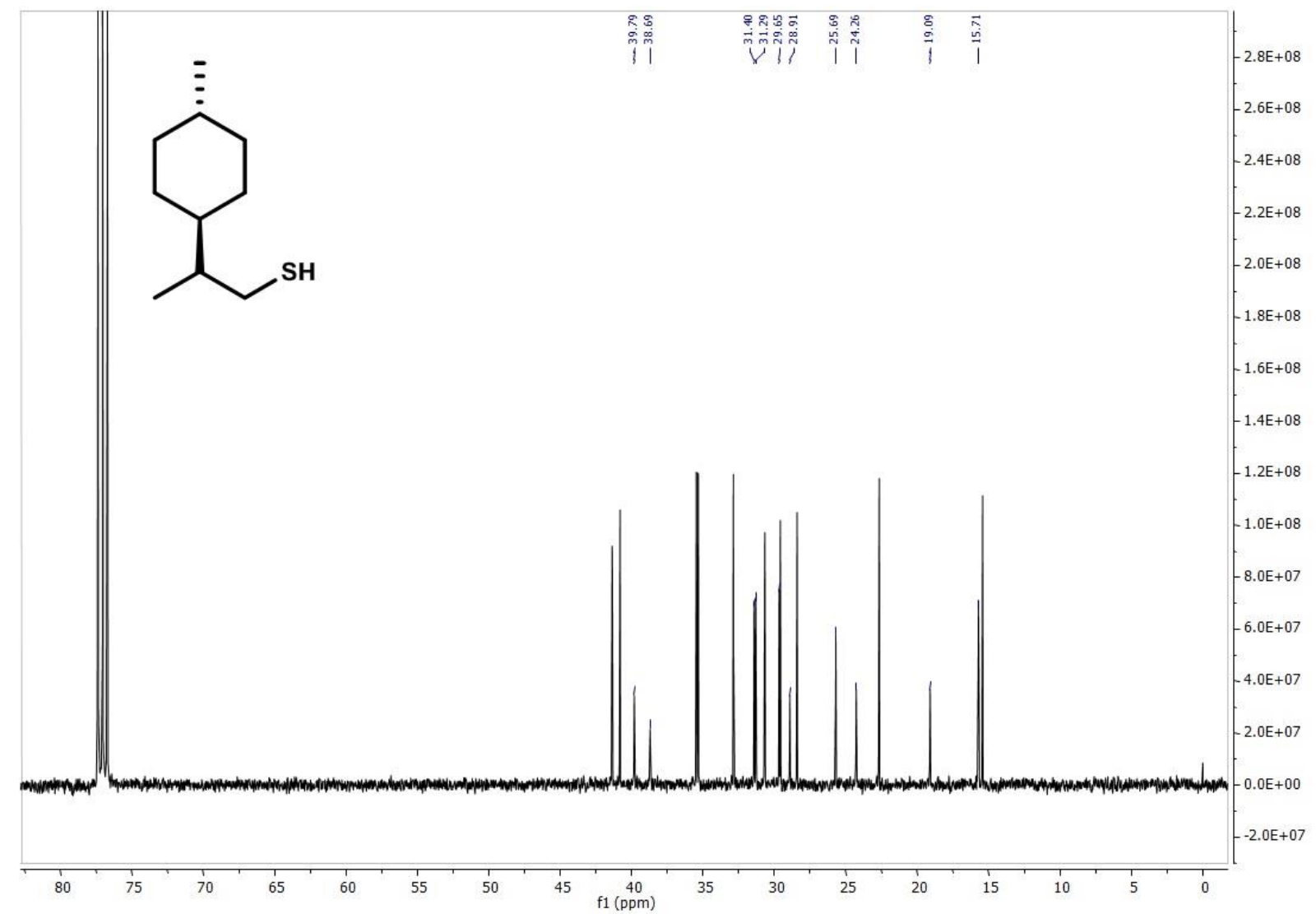

Figure S9. ${ }^{13} \mathrm{C}$ NMR spectrum of $p$-menthane-9-thiol (mixture of 2 diastereomers);

243 signals of diastereomer 2. 


\section{Compound 7}

245

246

$(1 R, 4 R)$-p-menthane-7-thiol

247

248

educt: (1R,4R)-4-isopropylcyclohexane-1-carboxylic acid

249

250

a) $1^{\text {st }}$ intermediate (obtained by reduction):

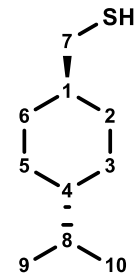

251

(1R,4R)-p-menthane-7-thiol, yield: $83 \%$,

252

MS-El: m/z (intensity in \%) 95 (100), 69 (24), 41 (18), 67 (18), 55 (16), 81 (14), 96

253

(10), 83 (9), 109 (9), 79 (8), 94 (7), 43 (7), 82 (6),57 (6), 123 (5), 138 (5), M+ absent

254

255

b) $2^{\text {nd }}$ intermediate:

256

(1R,4R)-4-(1-methylethyl)cyclohexyl)methyl 4-methylbenzenesulfonate,

257

crude yield: $64 \%$

258

259

c) $3^{\text {rd }}$ intermediate:

260

S-((1R,4R)-4-(1-methylethyl)cyclohexylmethyl) ethanethioate,

261 yield: $21 \%$,

262

MS-El: m/z (intensity in \%) 138 (100), 43 (85), 95 (54), 139 (38), 171 (38), 81 (37),

263

167 (35), 69 (38), 41 (23), 83 (22), 67 (20), 55 (17), 137 (16), 123 (15), 79 (14), 109

264

(14), 82 (12), 196 (11), 77 (8), 93 (8), 96 (8), 169 (8), 57 (7), 94 (7), 127 (6), 129 (6),

265

156 (5), 168 (5), $172(5), 181(5), 214\left(5, M^{+}\right)$ 


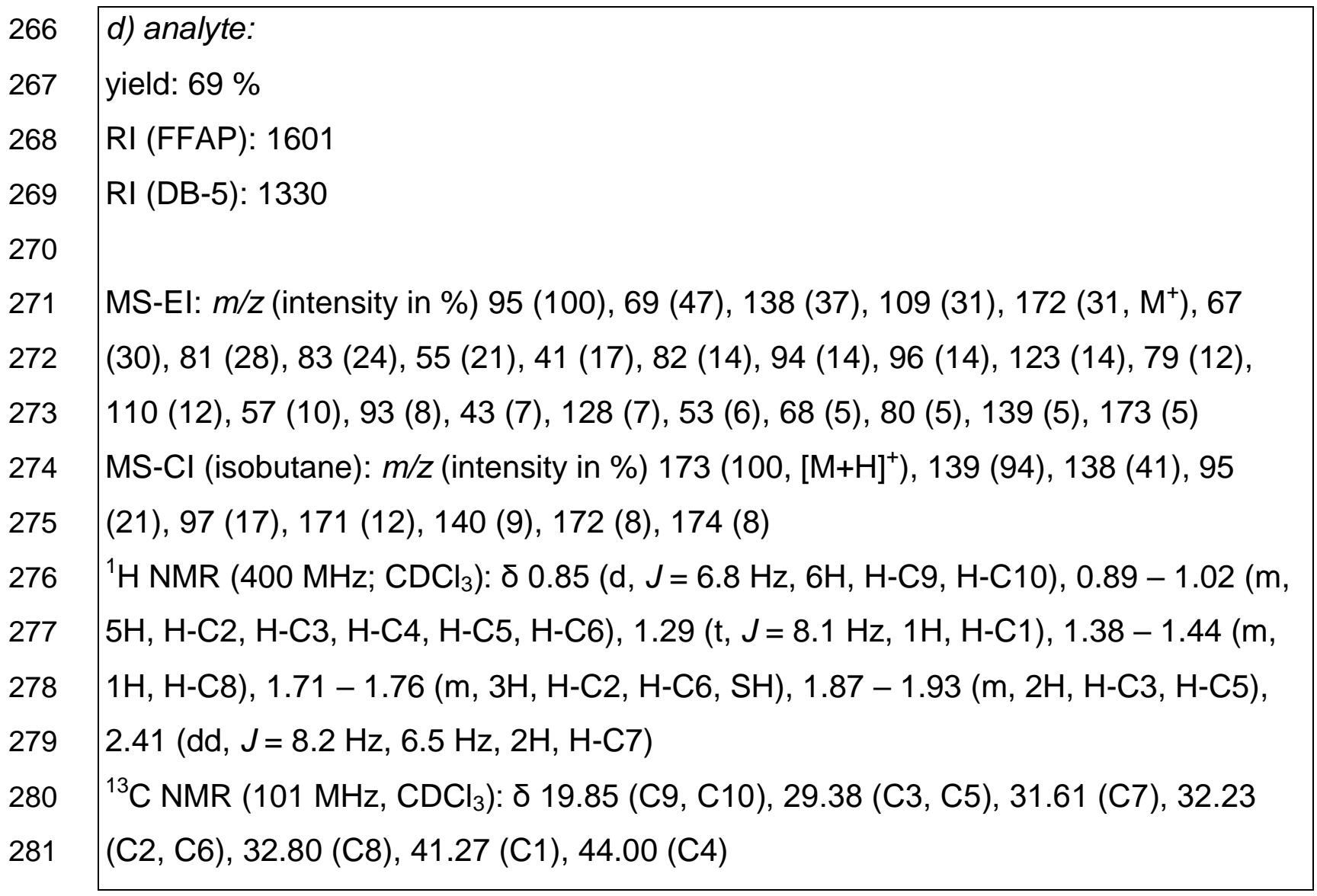




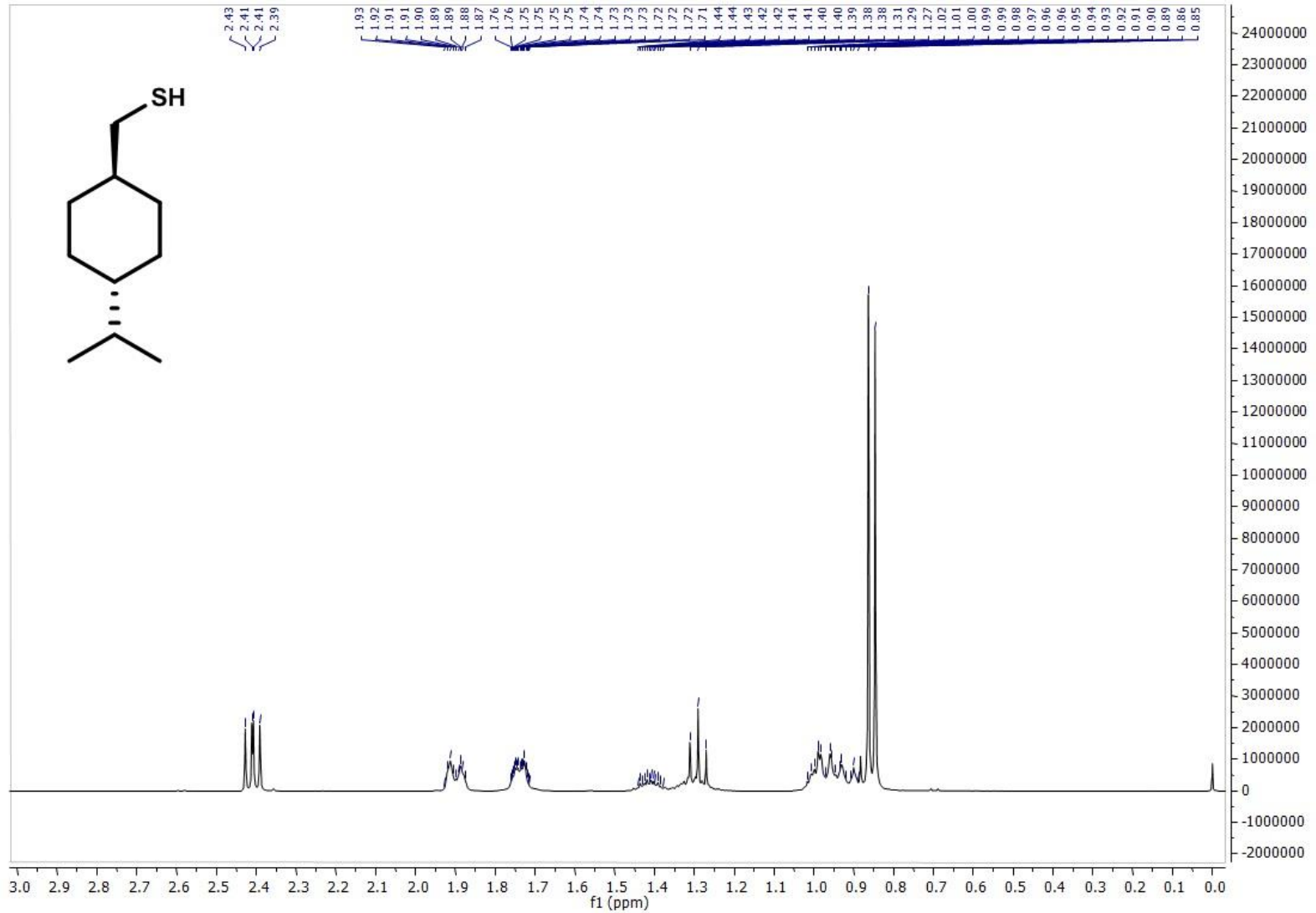

Figure S10. ${ }^{1} \mathrm{H}$ NMR spectrum of $(1 R, 4 R)-p$-menthane-7-thiol.

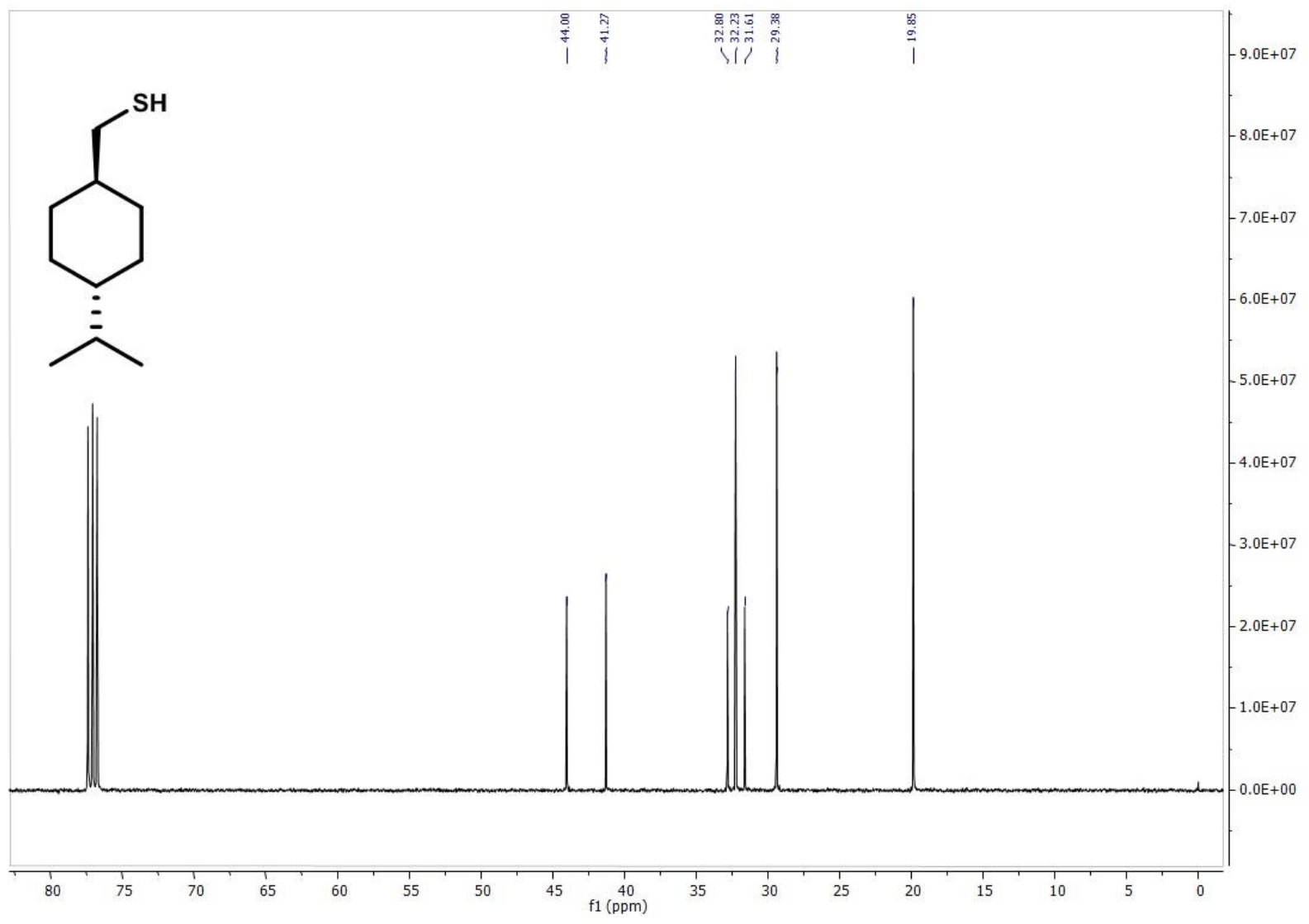

Figure $S 11 .{ }^{13} \mathrm{C}$ NMR spectrum of $(1 R, 4 R)$-p-menthane-7-thiol. 
288 Compound 8a

289

290

\section{$(1 R, 2 S, 4 R)-p$-menthane-2-thiol}

291

292

educt: (-)-dihydrocarveol

293

294

a) $1^{\text {st }}$ intermediate (obtained by hydrogenation):

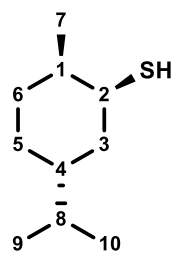

295

(-)-tetrahydrocarveol, yield: $90 \%$,

296

MS-El: m/z (intensity in \%) 95 (100), 57 (41), 55 (34), 113 (33), 43 (27), 81 (27), 41

297

(24), 82 (23), 69 (21), 67 (18), 83 (18), 138 (18), 96 (17), 123 (14), 109 (10), 70 (9),

298

$71(9), 68$ (8), 56 (7), 79 (7), 45 (6), 53 (6), 110 (6), M+ absent

299

300

b) $2^{\text {nd }}$ intermediate:

301

((1R,3R,6R)-6-methyl-3-(propan-2-yl)cyclohexyl) 4-methylbenzenesulfonate,

302

crude yield: $22 \%$

303

304

c) $3^{\text {rd }}$ intermediate:

305

S-((1S,3R,6R)-6-methyl-3-(propan-2-yl)cyclohexyl) ethanethioate,

306

yield: $74 \%$,

307

308

MS-El: m/z (intensity in \%) 138 (100), 95 (66), 83 (41), 81 (35), 43 (27), 55 (24), 69

309 (21), 67 (12), 82 (12), 96 (12), 123 (12), 139 (12), 41 (11), 57 (10), 137 (9), 94 (8), 97 (8), 79 (6), $109(5), 171(4), 214\left(3, \mathrm{M}^{+}\right)$ 


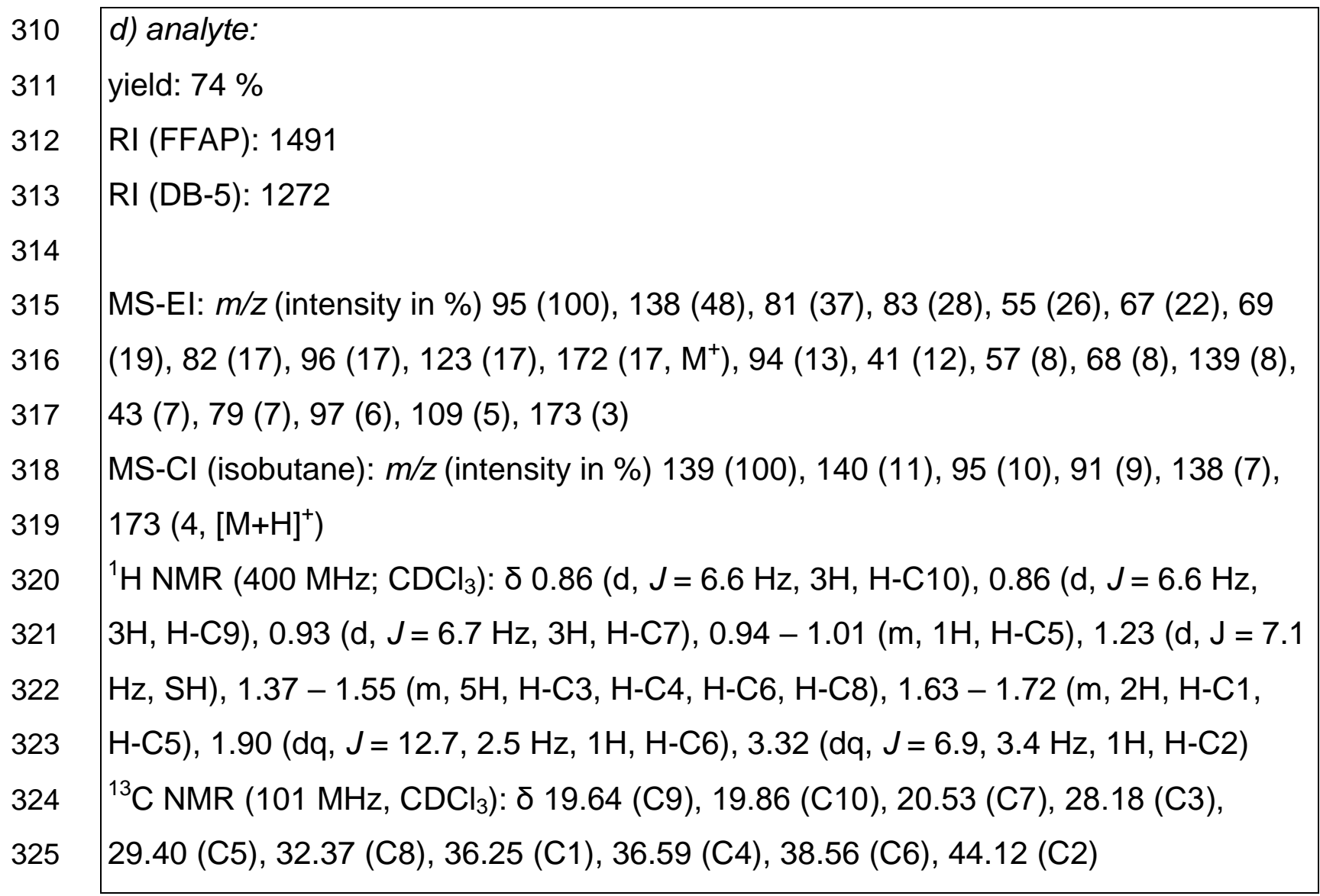




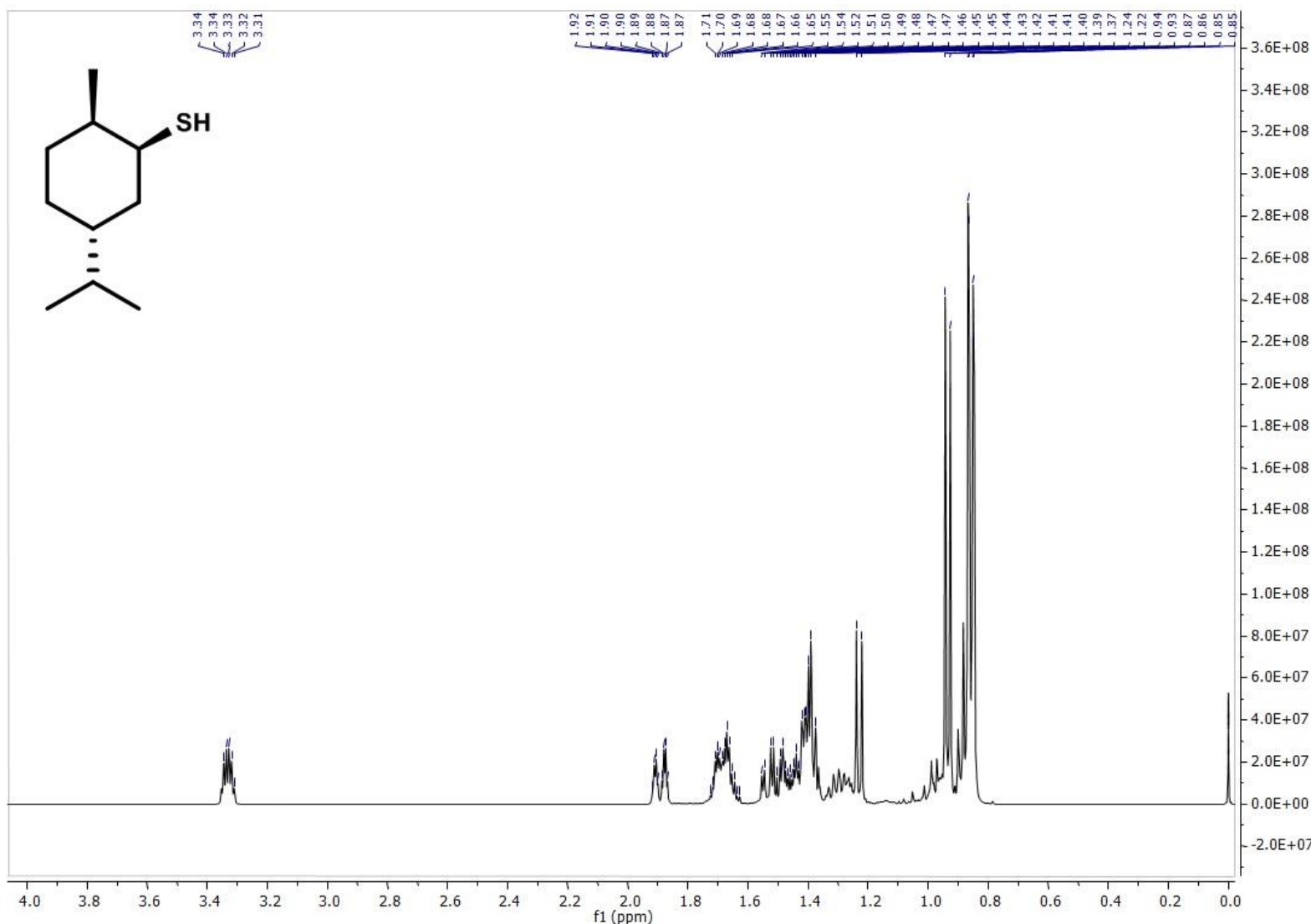

327 Figure S12. ${ }^{1} \mathrm{H}$ NMR spectrum of $(1 R, 2 S, 4 R)$-p-menthane-2-thiol.

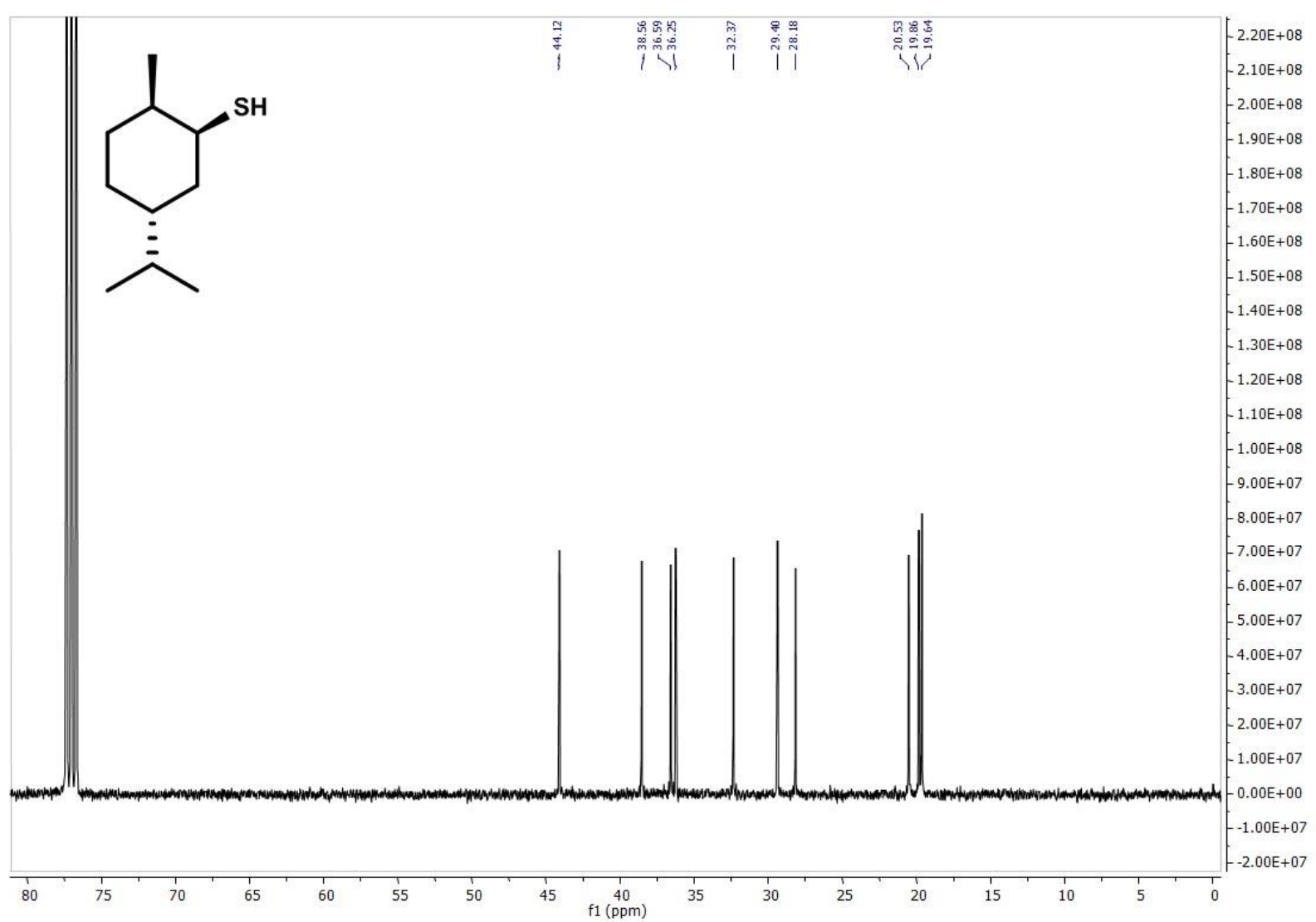

330 Figure $\mathrm{S13} .{ }^{13} \mathrm{C}$ NMR spectrum of $(1 R, 2 S, 4 R)-p$-menthane-2-thiol. 


\section{Compound $\mathbf{8 b}$}

332

333

334

335

educt: (+)-dihydrocarveol

336

337

a) $1^{\text {st }}$ intermediate (obtained by hydrogenation):

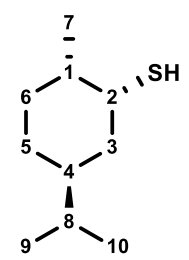

338

(+)-tetrahydrocarveol, yield: $86 \%$,

339

MS-El: $m / z$ (intensity in \%) 95 (100), 57 (36), 55 (35), 113 (34), 81 (25), 82 (24), 43

340

(23), 69 (23), 67 (22), 41 (20), 96 (19), 138 (18), 83 (16), 71 (11), 123 (11), 80 (9), 70

341

(8), 109 (8), 45 (6), 59 (6), 68 (6), 94 (6), 83 (5), $110(5), 156\left(1, \mathrm{M}^{+}\right)$

342

343

b) $2^{\text {nd }}$ intermediate:

344

((1S,3S,6S)-6-methyl-3-(propan-2-yl)cyclohexyl) 4-methylbenzenesulfonate,

345

crude yield: $45 \%$

346

347

c) $3^{\text {rd }}$ intermediate:

348

S-((1R,3S,6S)-6-methyl-3-(propan-2-yl)cyclohexyl) ethanethioate,

349 yield: $81 \%$,

350

351

352

MS-El: $m / z$ (intensity in \%) 138 (100), 95 (72), 83 (46), 81 (41), 43 (32), 55 (28), 69

(23), 41 (16), 67 (15), 96 (14), 123 (14), 139 (13), 57 (12), 82 (12), 94 (10), 97 (8), 79 (7), 68 (5), 80 (5), 109 (5), $171(4), 214\left(2, M^{+}\right)$ 


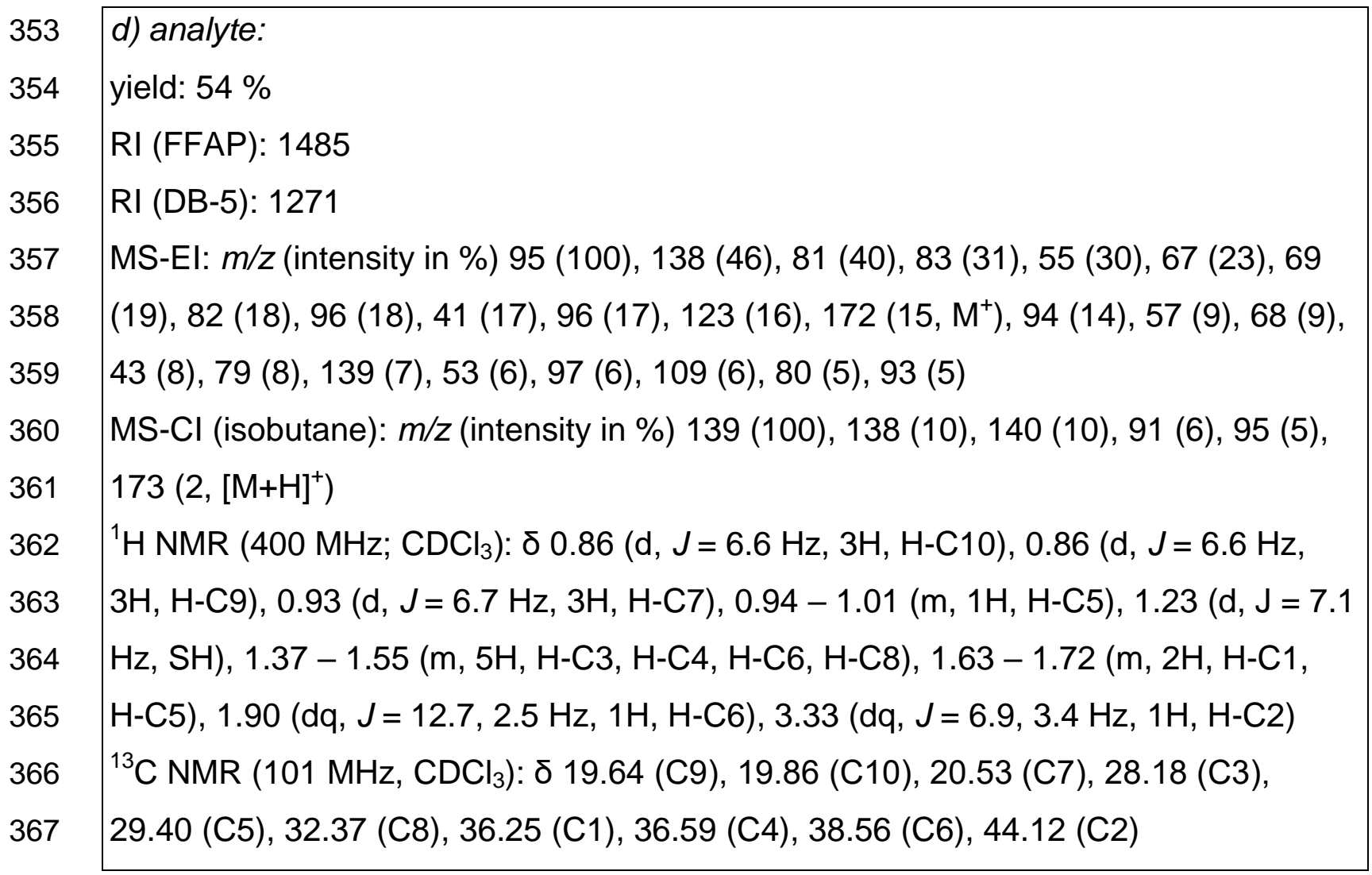




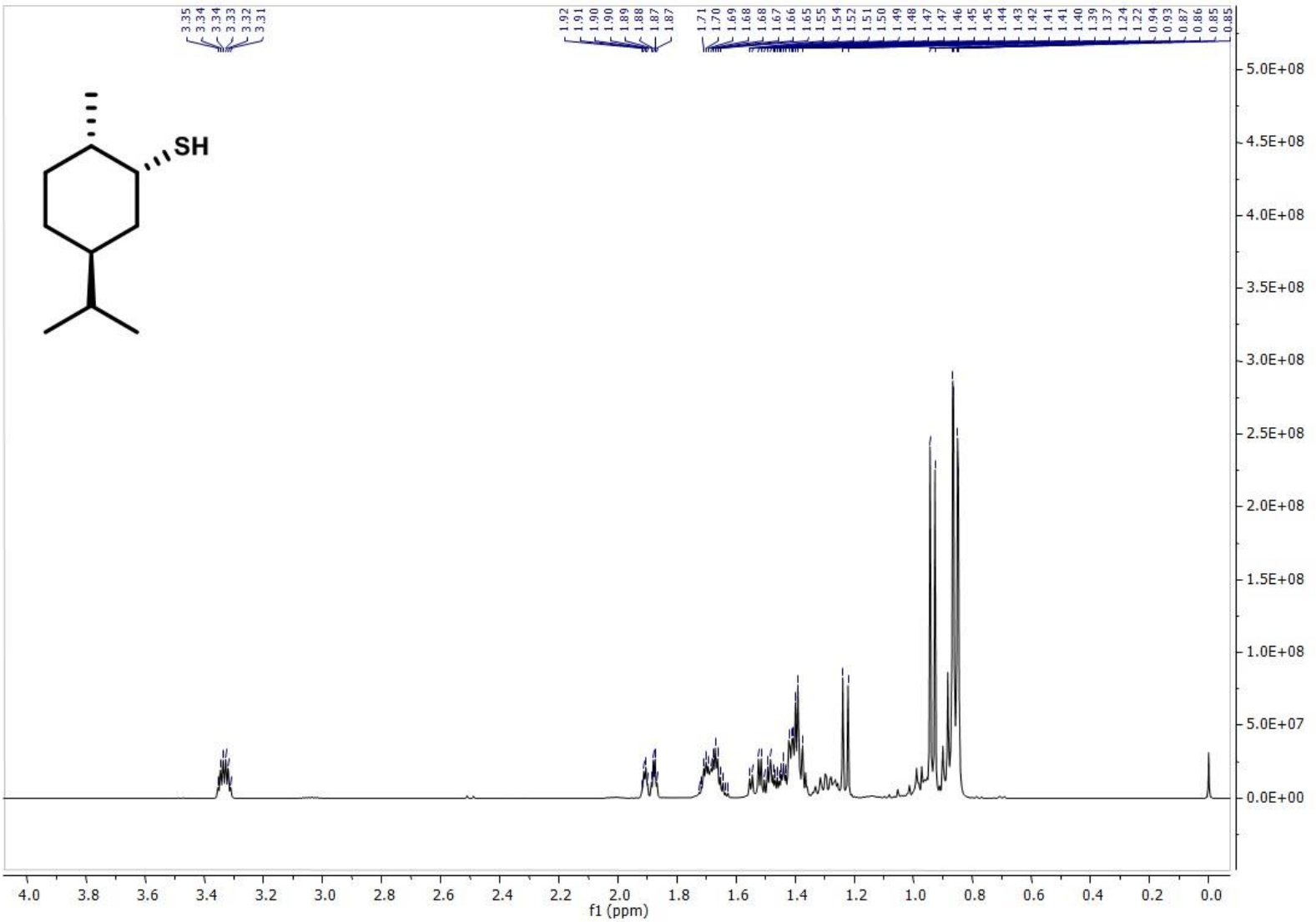

369 Figure S14. ${ }^{1} \mathrm{H}$ NMR spectrum of $(1 S, 2 R, 4 S)-p$-menthane-2-thiol.

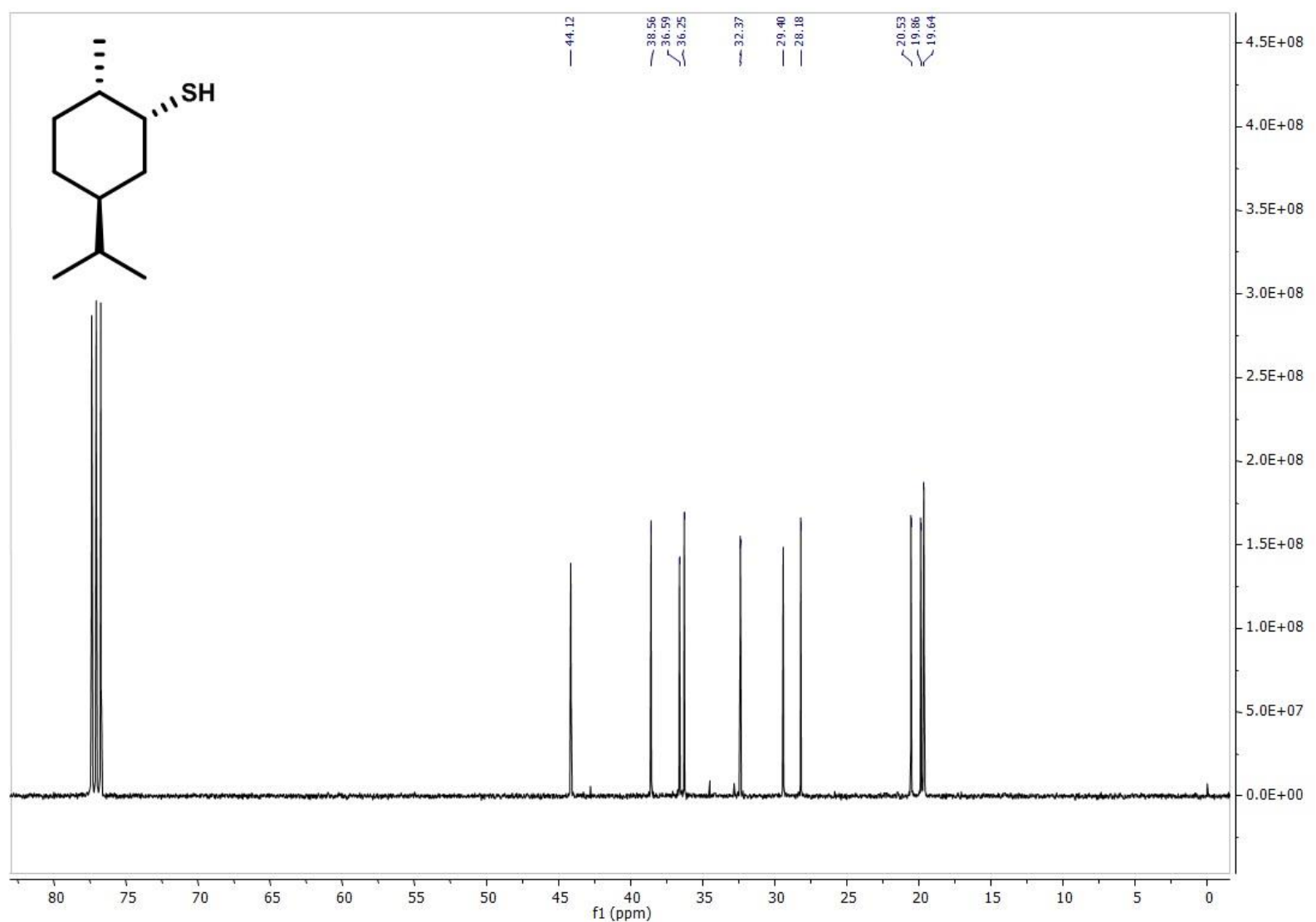

372 Figure S15. ${ }^{13} \mathrm{C}$ NMR spectrum of $(1 S, 2 R, 4 S)$-p-menthane-2-thiol. 
373 Compound 9a

374

375

376

377

378

379

380

381

382

383

384

385

386

387

388

389

390

391

392

393

a) $1^{\text {st }}$ intermediate:

\section{$(1 R, 3 S, 4 S)-p$-menthane-3-thiol}

educt: (-)-menthol

((1R,2S,5R)-5-methyl-2-(propan-2-yl)cyclohexyl) 4-methylbenzenesulfonate,

crude yield: $33 \%$

b) $2^{\text {nd }}$ intermediate:

S-((1S,2S,5R)-5-methyl-2-(propan-2-yl)cyclohexyl) ethanethioate, yield: $76 \%$,

MS-El: m/z (intensity in \%) 138 (100), 95 (85), 81 (59), 83 (50), 86 (38), 55 (31), 69

(25), 67 (20), 123 (16), 96 (16), 82 (16), 139 (15), 134 (14), 57 (12), 97 (11), 94 (10), $79(9), 128(7), 171(6), 214\left(1, M^{+}\right)$

c) analyte:

yield: $77 \%$

RI (FFAP): 1461

RI (DB-5): 1241

394

395

MS-El: $m / z$ (intensity in \%) 95 (100), 81 (38), 138 (36), 123 (23), 55 (22), 83 (22), 67 396 (19), 96 (19), 69 (15), 82 (14), $172\left(14, M^{+}\right), 94$ (8), 68 (7), 139 (6), 79 (5), 119 (5) 397 398 MS-Cl (isobutane): $m / z$ (intensity in \%) $139(100), 140(9), 138(6), 173\left(1,[\mathrm{M}+\mathrm{H}]^{+}\right)$ ${ }^{1} \mathrm{H}$ NMR $\left(400 \mathrm{MHz} ; \mathrm{CDCl}_{3}\right): \delta 0.80-0.86(\mathrm{~m}, 1 \mathrm{H}, \mathrm{H}-\mathrm{C} 6), 0.86(\mathrm{~d}, J=6.3 \mathrm{~Hz}, 3 \mathrm{H}, \mathrm{H}-$ 399 400 C7), 0.90 (d, $J=3.3 \mathrm{~Hz}, 3 \mathrm{H}, \mathrm{H}-\mathrm{C} 10$ ), 0.91 (d, $J=3.3 \mathrm{~Hz}, 3 \mathrm{H}, \mathrm{H}-\mathrm{C} 10$ ), 1.03 (ddt, $J=$ 401 12.4, 9.6, 3.3 Hz, 1H, H-C4), 1.22 (d, J = 6.9 Hz, SH), 1.25 - $1.33(\mathrm{~m}, 1 \mathrm{H}, \mathrm{H}-\mathrm{C} 5)$, 1.39 (ddd, J = 13.0, 11.7, 3.8 Hz, 1H, H-C2), $1.44-1.53$ (m, 1H, H-C8) , $1.65-1.89$ 402 (m, 4H, H-C1, H-C2, H-C5, H-C6), $3.48-3.51$ (m, 1H, H-C3) 403 404 ${ }^{13} \mathrm{C} \mathrm{NMR} \mathrm{(101} \mathrm{MHz,} \mathrm{CDCl} 3$ ): $\delta 20.41$ (C9), 20.90 (C10), 22.19 (C7), 24.22 (C5), 25.98 (C1), 30.35 (C8), 35.33 (C6), 40.22 (C3), 44.07 (C2), 48.29 (C4) 


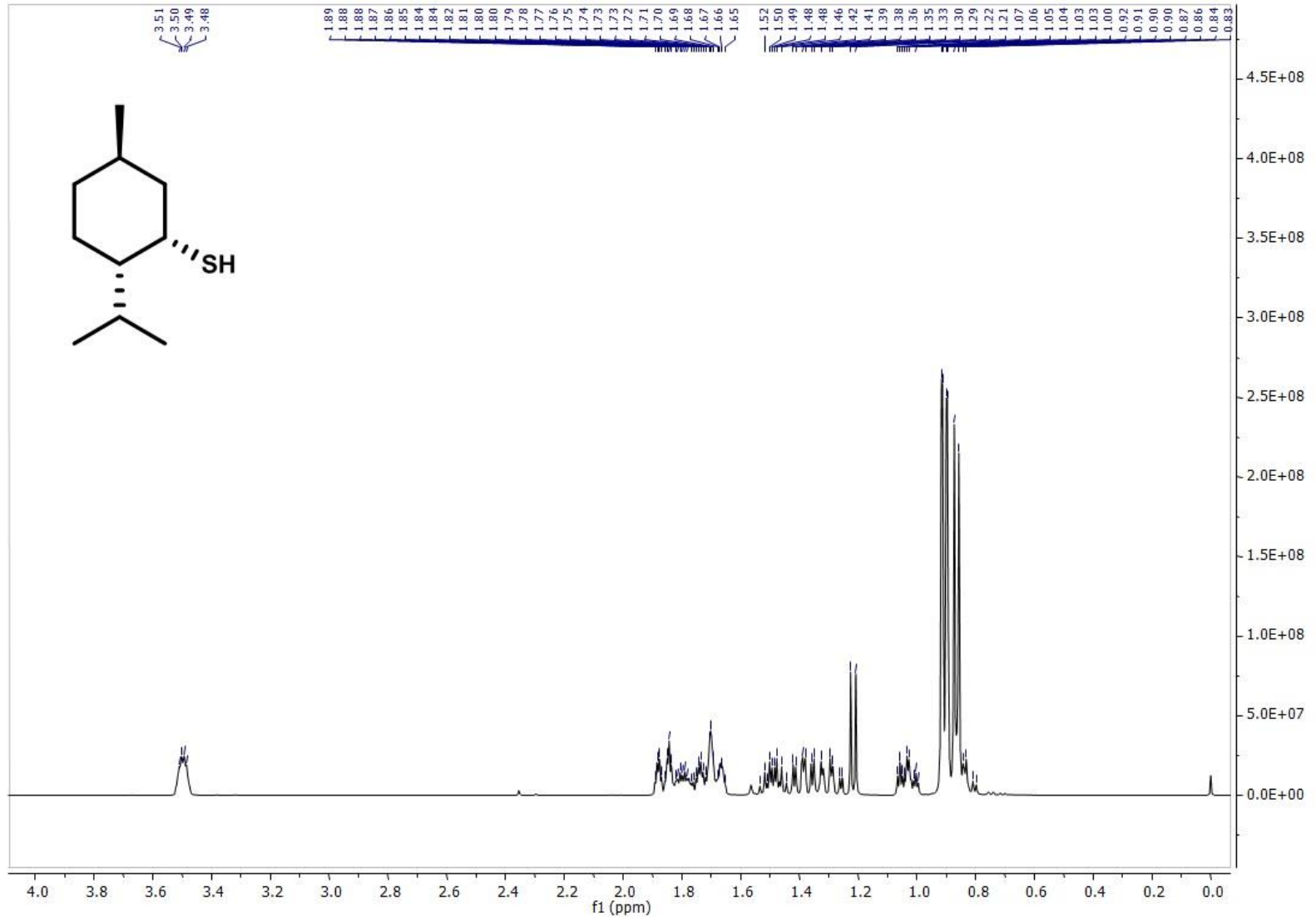

406 Figure S16. ${ }^{1} \mathrm{H}$ NMR spectrum of $(1 R, 3 S, 4 S)-p$-menthane-3-thiol.

407

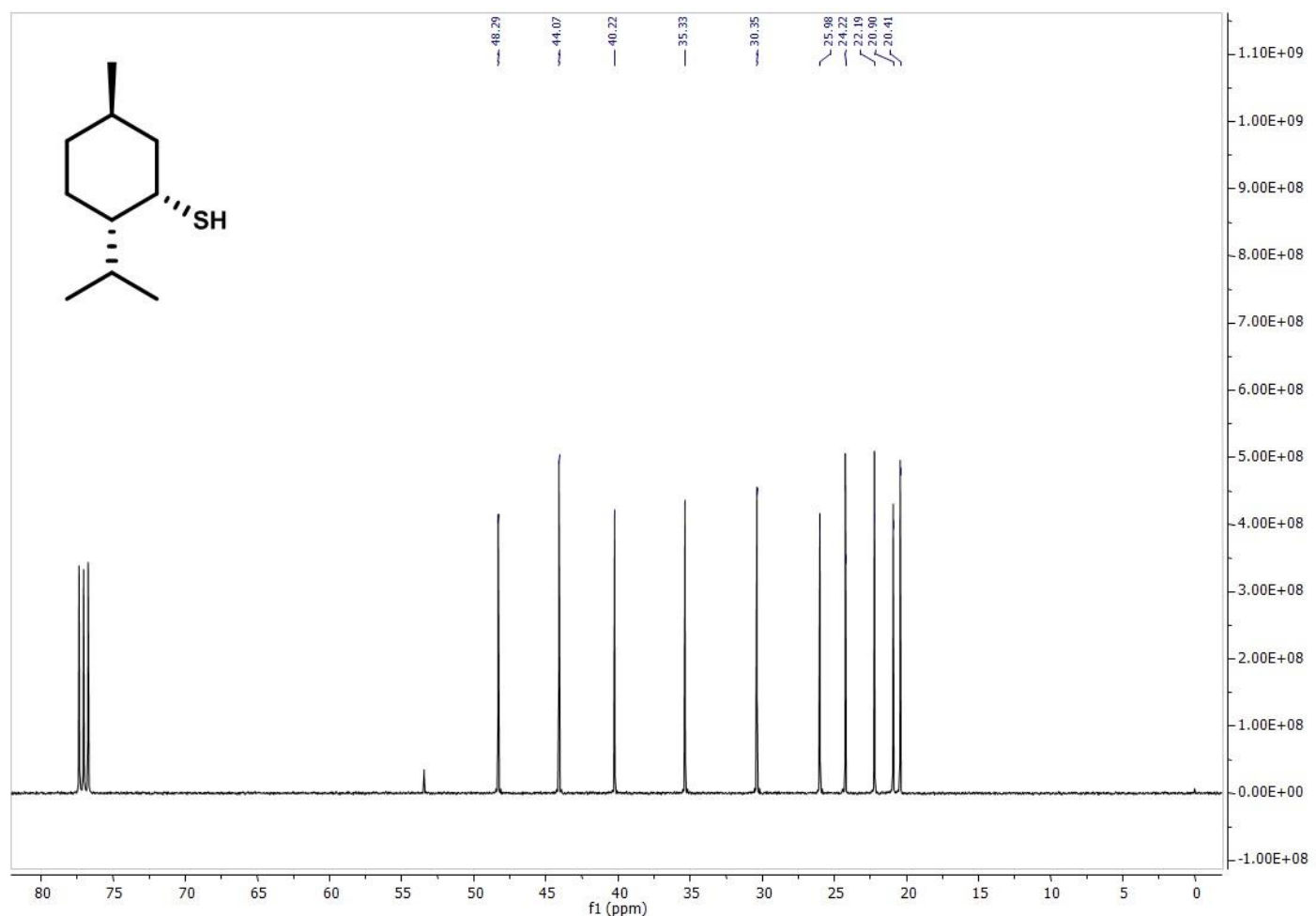

409 Figure S17. ${ }^{13} \mathrm{C}$ NMR spectrum of $(1 R, 3 S, 4 S)$-p-menthane-3-thiol. 
410 Compound 9b

411

412

413

414

415

416

417

418

419

420

421

422

423

424

425

426

427

428

429

430

a) $1^{\text {st }}$ intermediate:

\section{$(1 R, 3 R, 4 R)-p$-menthane-3-thiol}

educt: (+)-isomenthol

((1S,2R,5R)-5-methyl-2-(propan-2-yl)cyclohexyl) 4-methylbenzenesulfonate, crude yield: $38 \%$

b) $2^{\text {nd }}$ intermediate:

S-((1R,2R,5R)-5-methyl-2-(propan-2-yl)cyclohexyl) ethanethioate, yield: $64 \%$,

MS-El: $m / z$ (intensity in \%) 138 (100), 95 (91), 81 (64), 83 (57), 86 (41), 55 (34), 69 (28), 67 (23), 123 (19), 96 (18), 139 (18), 82 (17), 134 (13), 57 (13), 97 (13), 79 (12), $94(11), 128(8), 171(7), 214\left(3, M^{+}\right)$

c) analyte:

yield: $59 \%$

RI (FFAP): 1520

RI (DB-5): 1274

431

432

MS-El: $m / z$ (intensity in \%) 95 (100), 81 (62), 55 (43), 83 (39), 123 (32), 67 (31), 69

433 (28), 138 (28), $172\left(27, \mathrm{M}^{+}\right), 96$ (22), 82 (19), 139 (12), 57 (11), 79 (11), 53 (9), 94

434

(9), 93 (8), 109 (7), 77 (6), 87 (5), 91 (5)

435

MS-Cl (isobutane): $m / z$ (intensity in \%) $139(100), 140(13), 138(5), 173\left(1,[\mathrm{M}+\mathrm{H}]^{+}\right)$

436

${ }^{1} \mathrm{H}$ NMR $\left(400 \mathrm{MHz} ; \mathrm{CDCl}_{3}\right): \delta 0.94(\mathrm{~d}, J=3.3 \mathrm{~Hz}, 3 \mathrm{H}, \mathrm{H}-\mathrm{C}$ ) $), 0.96(\mathrm{~d}, J=3.3 \mathrm{~Hz}, 3 \mathrm{H}$,

437

438 $\mathrm{H}-\mathrm{C} 10), 1.08$ (d, J = 7.1 Hz, 3H, H-C7), $1.20-1.26$ (m, 1H, H-C4), $1.33-1.41$ (m, $1 \mathrm{H}, \mathrm{H}-\mathrm{C} 6), 1.43(\mathrm{~d}, J=6.4 \mathrm{~Hz}, \mathrm{SH}), 1.45-1.55(\mathrm{~m}, 2 \mathrm{H}, \mathrm{H}-\mathrm{C} 5, \mathrm{H}-\mathrm{C} 6), 1.59-1.83(\mathrm{~m}$, 439 4H, H-C1, H-C2, H-C5, H-C8), $1.91-1.97$ (m, 1H, H-C2), $3.40-3.45$ (m, 1H, H-C3) 440

${ }^{13} \mathrm{C} \mathrm{NMR}\left(101 \mathrm{MHz}, \mathrm{CDCl}_{3}\right): \delta 21.17$ (C9), 21.81 (C10), 21.88 (C7), 22.33 (C5), 28.96 (C8), 29.31 (C1), 31.52 (C6), 39.95 (C3), 40.94 (C2), 47.32 (C4) 


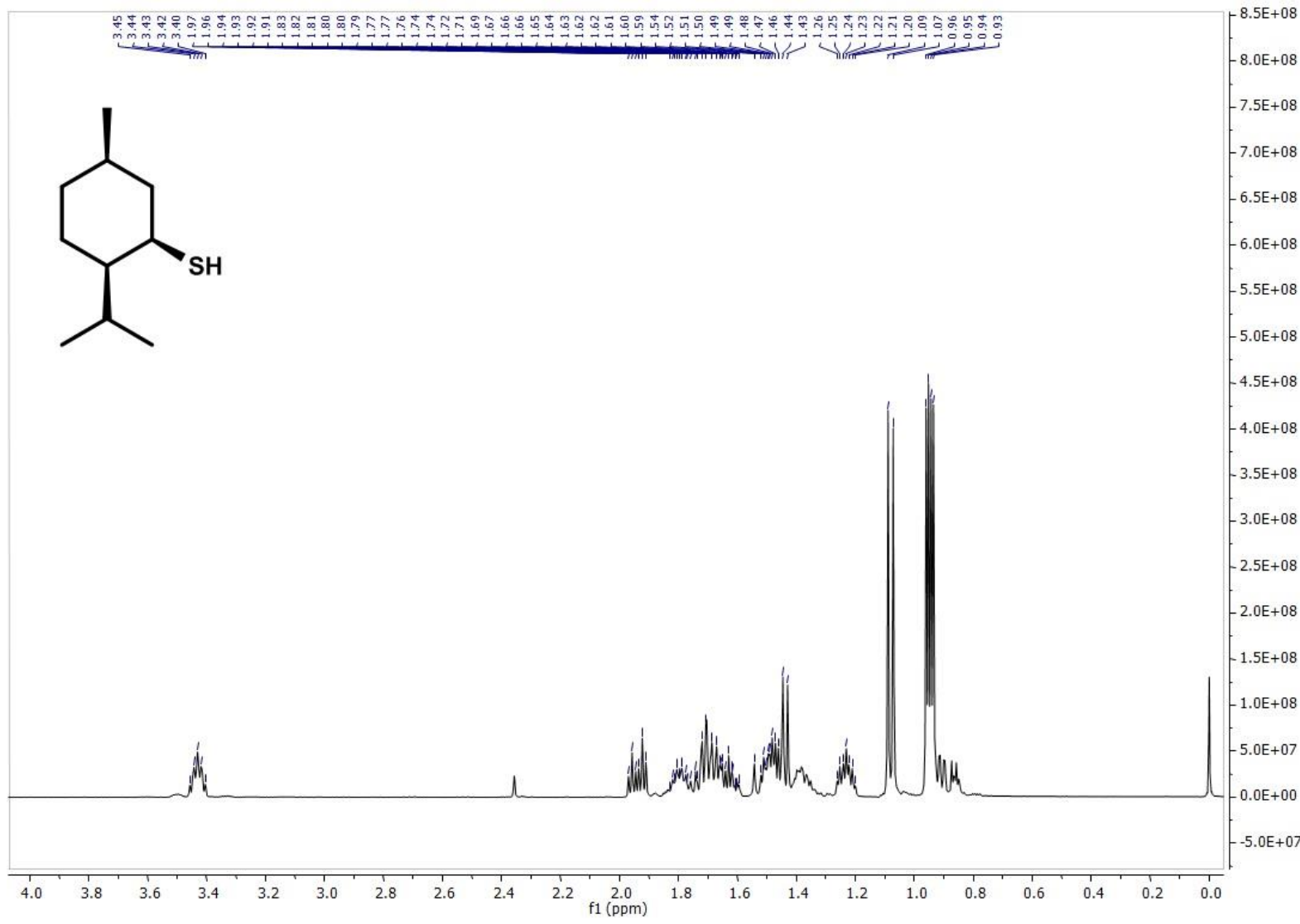

443 Figure S18. ${ }^{1} \mathrm{H}$ NMR spectrum of $(1 R, 3 R, 4 R)-p$-menthane-3-thiol.

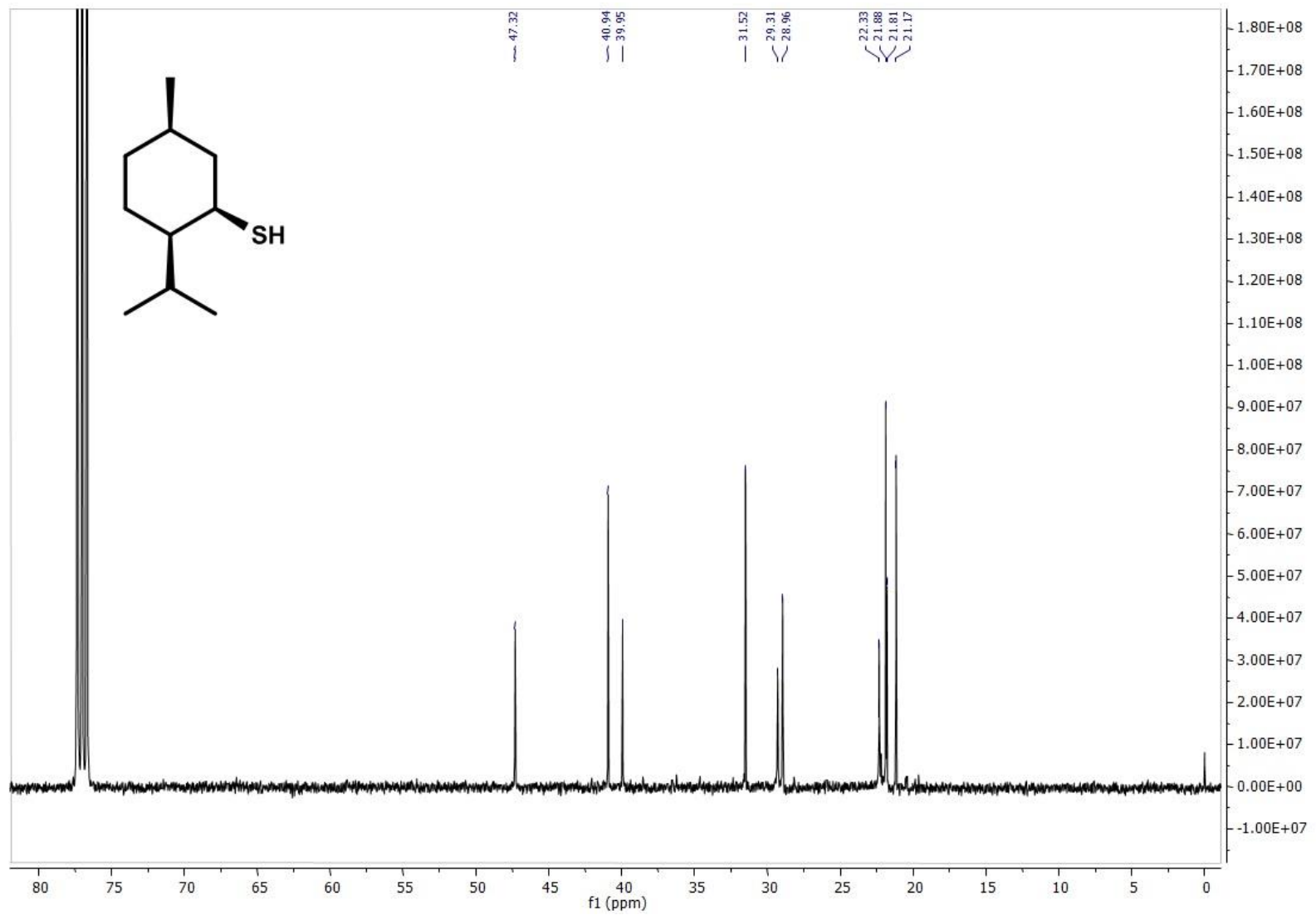

446 Figure S19. ${ }^{13} \mathrm{C}$ NMR spectrum of $(1 R, 3 R, 4 R)$-p-menthane-3-thiol. 
447 Compound 9c

448

449

\section{$(1 S, 3 R, 4 R)$-p-menthane-3-thiol}

450

451

educt: (+)-menthol

452

453

a) $1^{\text {st }}$ intermediate:

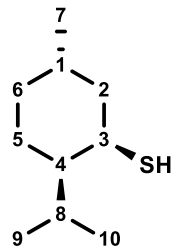

454

((1S,2R,5S)-5-methyl-2-(propan-2-yl)cyclohexyl) 4-methylbenzenesulfonate,

455

crude yield: $30 \%$

456

457

b) $2^{\text {nd }}$ intermediate:

458

S-((1R,2R,5S)-5-methyl-2-(propan-2-yl)cyclohexyl) ethanethioate,

459

yield: $68 \%$

460

MS-El: m/z (intensity in \%) 95 (100), 138 (100), 81 (75), 83 (65), 86 (62), 69 (32), 55

461

(31), 67 (27), 123 (20), 84 (20), 82 (19), 96 (18), 49 (18), 139 (15), 137 (14), 97 (13),

462

57 (13), 94 (13), $109(8), 171(5), 214\left(2, \mathrm{M}^{+}\right)$

463

464

c) analyte:

465

yield: $74 \%$

466

RI (FFAP): 1461

467

RI (DB-5): 1241

468

469

MS-El: m/z (intensity in \%) 95 (100), 138 (61), 81 (60), 67 (34), $172\left(32, \mathrm{M}^{+}\right), 83(30)$,

470

55 (29), 123 (29), 96 (21), 82 (21), 69 (18), 94 (17), 79 (12), 68 (11), 139 (9), 57 (9),

471

80 (8), 109 (8), 53 (7), 97 (7), 93 (6)

472

MS-Cl (isobutane): m/z (intensity in \%) 139 (100), $140(10), 138(7), 173\left(4,[\mathrm{M}+\mathrm{H}]^{+}\right)$

473

${ }^{1} \mathrm{H}$ NMR (400 MHz; $\left.\mathrm{CDCl}_{3}\right): \delta 83-0.91(\mathrm{~m}, 1 \mathrm{H}, \mathrm{H}-\mathrm{C} 6), 0.86(\mathrm{~d}, \mathrm{~J}=6.4 \mathrm{~Hz}, 3 \mathrm{H}, \mathrm{H}-$

474

C7), 0.90 (d, J=3.3 Hz, 3H, H-C9), 0.91 (d, 3.3 Hz, 3H, H-C10), 1.03 (ddt, J= 12.5,

475

9.6, 3.3 Hz, 1H, H-C4), 1.22 (d, J=7.0 Hz, SH), $1.25-1.33$ (m, 1H, H-C5), 1.39

476

(ddd, $J=13.1,11.7,3.8 \mathrm{~Hz}, 1 \mathrm{H}, \mathrm{H}-\mathrm{C} 2), 1.44-1.53$ (m, 1H, H-C8) , 1.65 - 1.89 (m,

477

$4 \mathrm{H}, \mathrm{H}-\mathrm{C} 1, \mathrm{H}-\mathrm{C} 2, \mathrm{H}-\mathrm{C} 3, \mathrm{H}-\mathrm{C} 5, \mathrm{H}-\mathrm{C} 6), 3.48-3.52$ (m, 1H, H-C3)

478

${ }^{13} \mathrm{C}$ NMR (101 MHz, $\mathrm{CDCl}_{3}$ ): $\delta 20.41$ (C9), 20.90 (C10), 22.18 (C7), 24.21 (C5),

479

25.98 (C1), 30.36 (C8), 35.33 (C6), 40.22 (C3), 44.07 (C2), 48.29 (C4) 


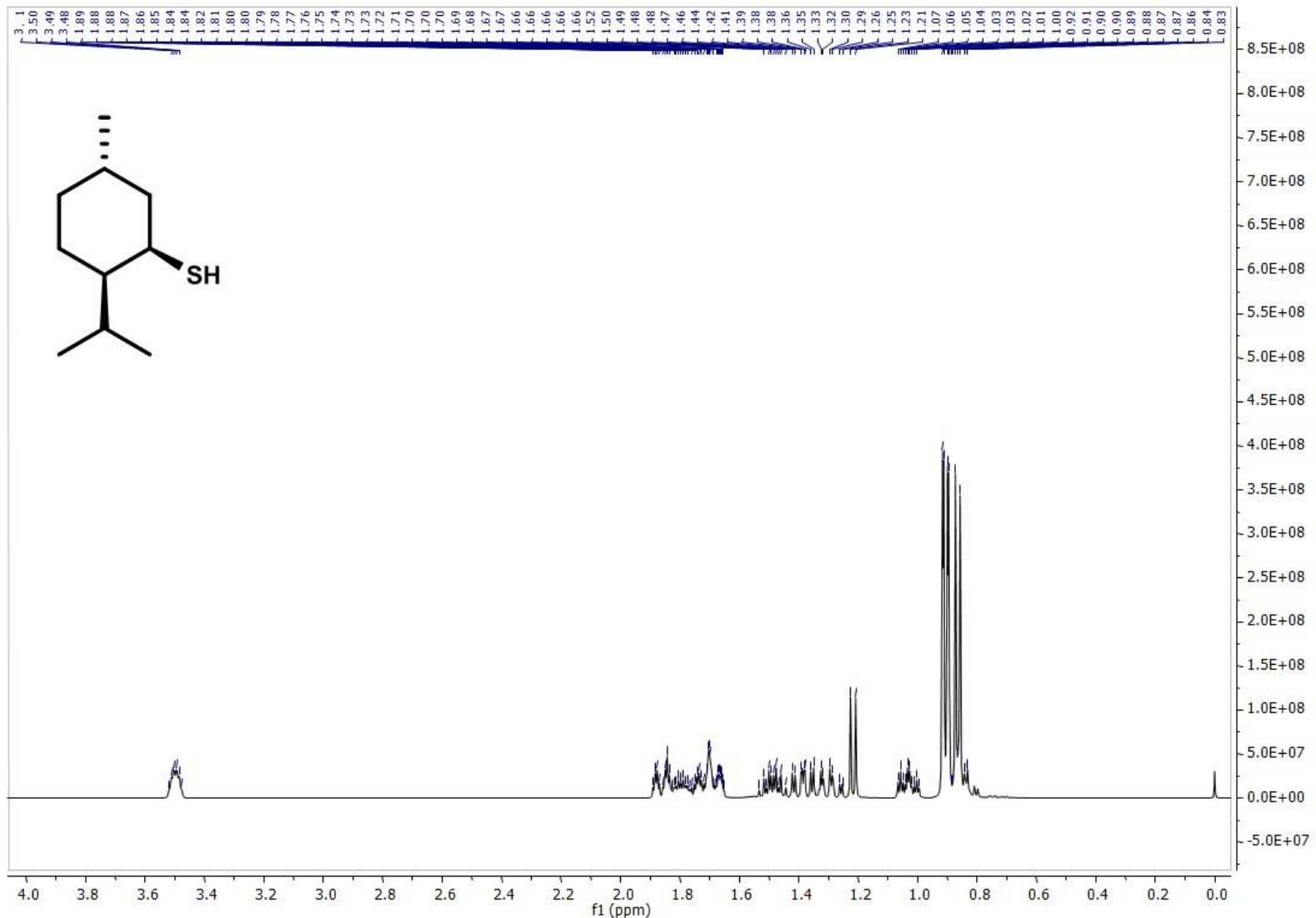

481 Figure S20. ${ }^{1} \mathrm{H}$ NMR spectrum of $(1 S, 3 R, 4 R)-p$-menthane-3-thiol.

482

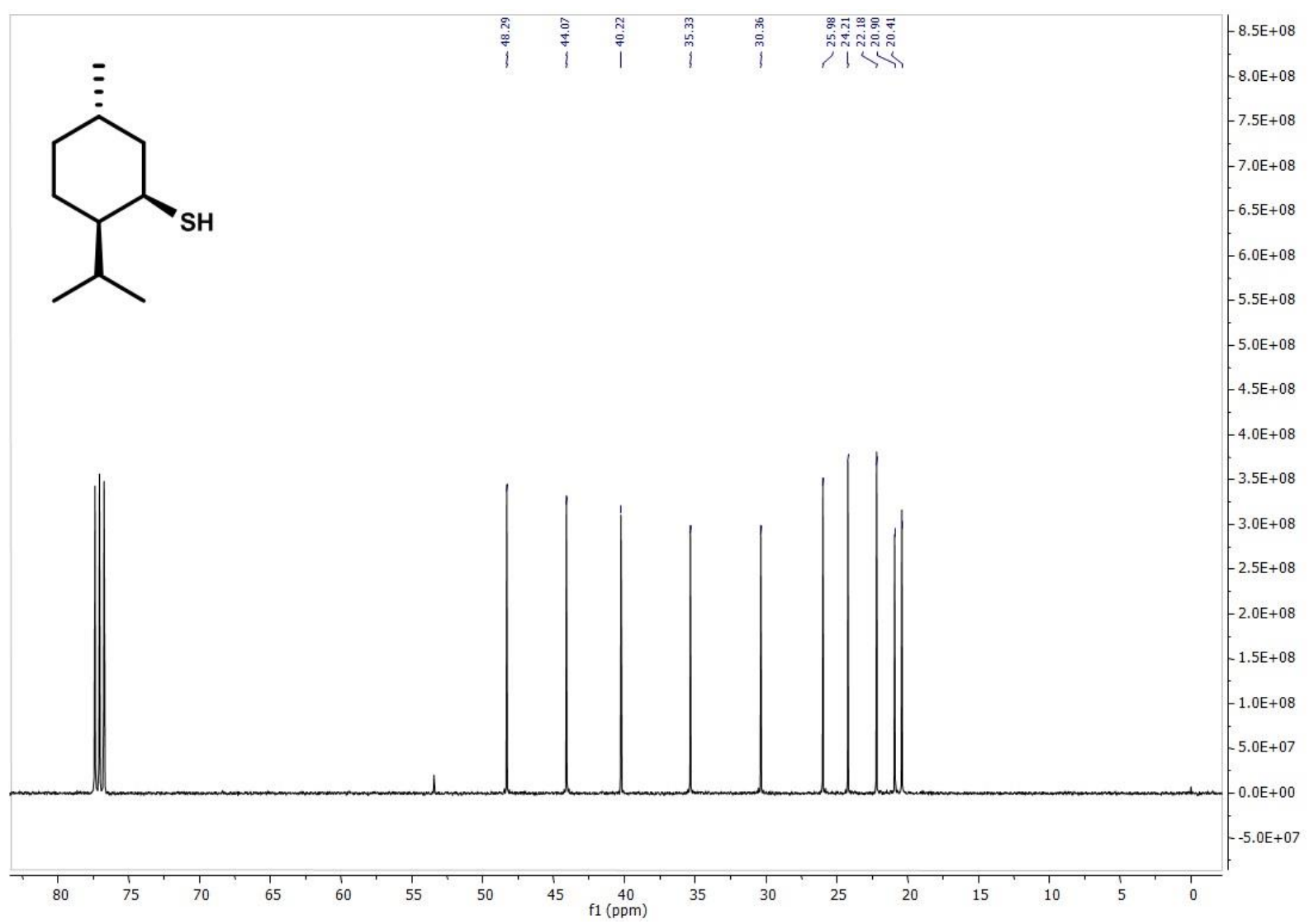

Figure $521 .{ }^{13} \mathrm{C}$ NMR spectrum of $(1 S, 3 R, 4 R)$-p-menthane-3-thiol. 
485 Compound 12

486

487

p-cymene-9-thiol

488

489

educt: $p$-cymene-9-ol

490

491

a) $1^{\text {st }}$ intermediate:

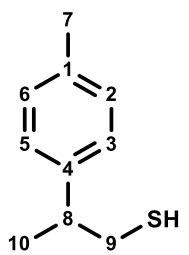

492

2-(p-tolyl)propyl 4-methylbenzenesulfonate,

493

crude yield: $52 \%$

494

495

b) $2^{\text {nd }}$ intermediate:

496

S-(2-p-tolylpropyl) ethanethioate,

497

yield: $78 \%$,

498

MS-El: m/z (intensity in \%) 119 (100), 132 (89), 117 (32), 91 (26), 115 (15), 133 (10),

499

120 (9), 77 (8), 43 (7), 65 (7), 118 (6), 103 (5), 105 (5), 116 (5), $208\left(2, \mathrm{M}^{+}\right)$

500

501

c) analyte:

502

yield: $95 \%$

503

RI (FFAP): 1813

504

RI (DB-5): 1324

505

506

MS-El: m/z (intensity in \%) 119 (100), 91 (27), $117(20), 166\left(17, \mathrm{M}^{+}\right), 120$ (12), 115

507

(8), 77 (6), 65 (5), 103 (5)

508

MS-Cl (isobutane): $m / z$ (intensity in \%) 133 (100), 134 (12), 119 (11), 166 (4), 167 (3,

509

$\left.[\mathrm{M}+\mathrm{H}]^{+}\right)$

510

511

512

${ }^{1} \mathrm{H}$ NMR (400 MHz; $\left.\mathrm{CDCl}_{3}\right): \delta 1.22$ (dd, $\left.J=7.2,9.0 \mathrm{~Hz}, \mathrm{SH}\right), 1.33(\mathrm{~d}, J=6.9 \mathrm{~Hz}, 3 \mathrm{H}$, $\mathrm{H}-\mathrm{C} 10), 2.33$ (s, 3H, H-C7), $2.63-2.70$ (m, 1H, H-C9), $2.74-2.81$ (m, 1H, H-C9),

2.86 (h, J=6.8 Hz, 1H, H-C8), 7.09 (d, J=8.2 Hz, 2H, H-C3, H-C5), 7.13 (d, J=8.6

513

$\mathrm{Hz}, 2 \mathrm{H}, \mathrm{H}-\mathrm{C} 2, \mathrm{H}-\mathrm{C} 6)$

514

${ }^{13} \mathrm{C}$ NMR (101 MHz, $\mathrm{CDCl}_{3}$ ): $\delta 20.80$ (C10), 21.04 (C7), 32.66 (C9), 42.93 (C8),

127.04 (C3, C5), 129.22 (C2, C6), 136.13 (C1), 141.84 (C4) 


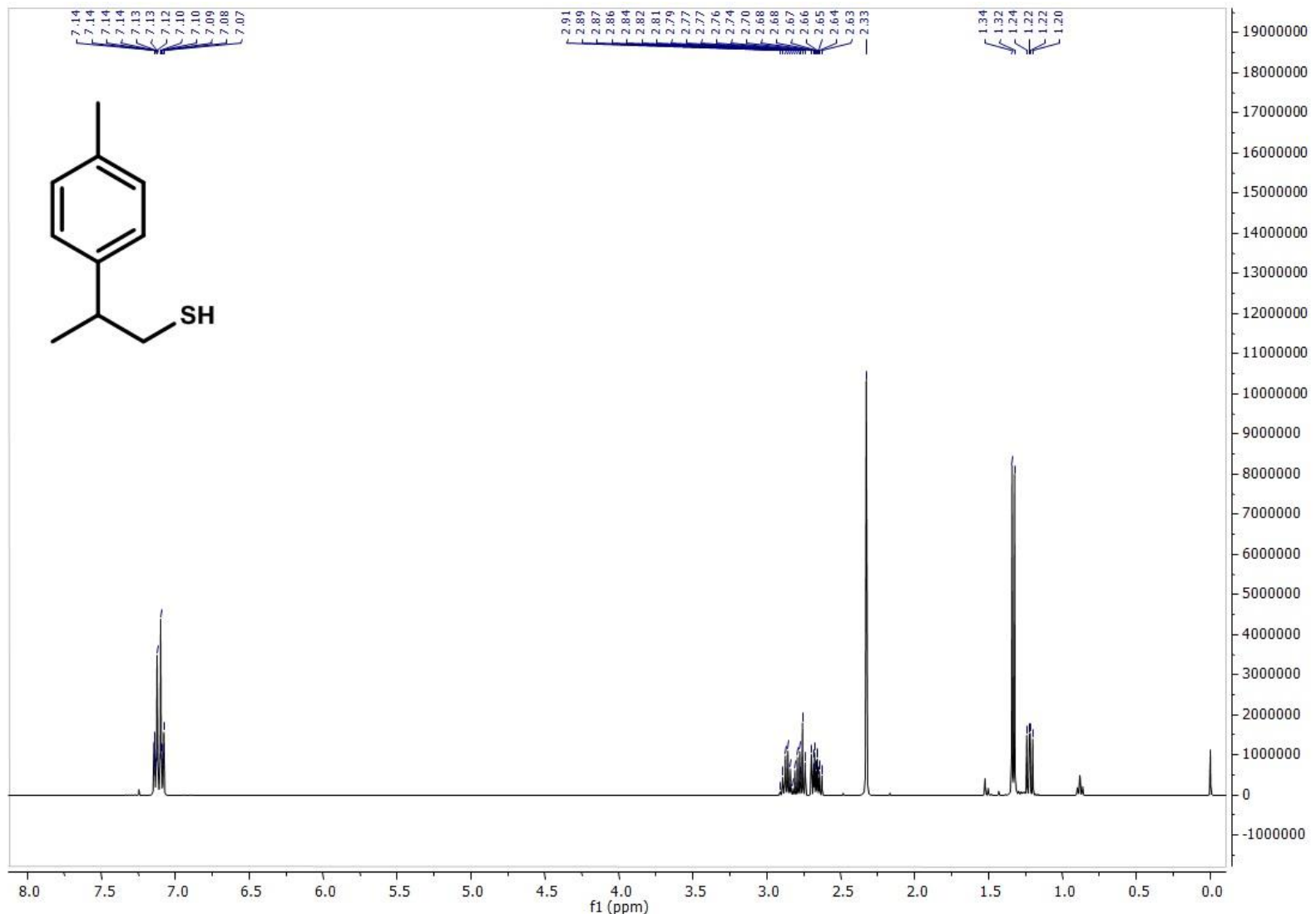

517 Figure S22. ${ }^{1} \mathrm{H}$ NMR spectrum of $p$-cymene-9-thiol.

518

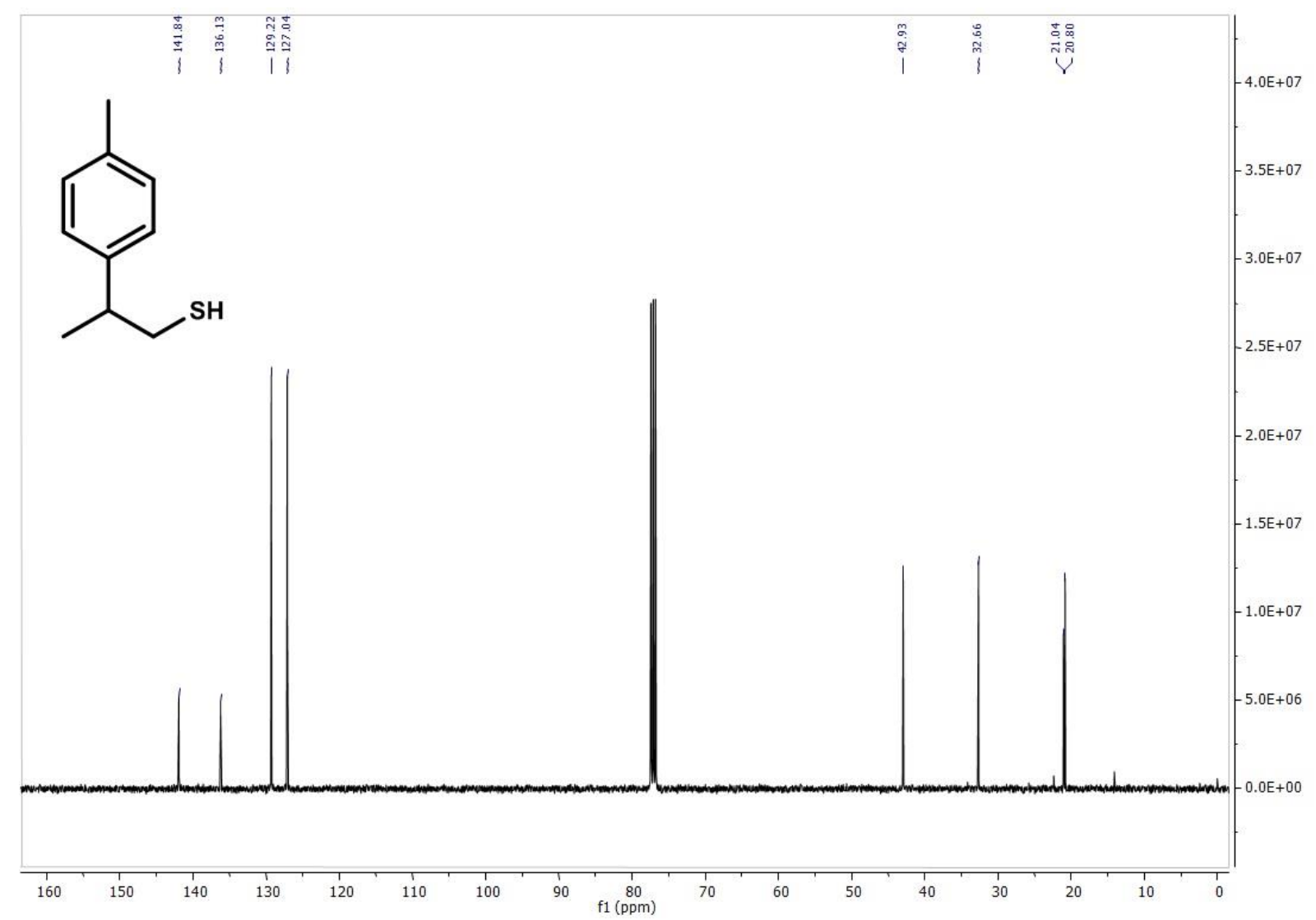

Figure S23. ${ }^{13} \mathrm{C}$ NMR spectrum of $p$-cymene-9-thiol. 
Compound 15a

522

523

\section{$(1 R, 2 S, 4 R)-8-p$-menthene-2-thiol}

524

525

educt: (-)-dihydrocarveol

526

527

a) $1^{\text {st }}$ intermediate:

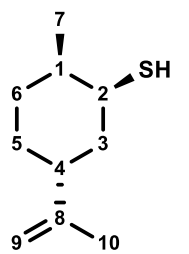

528

529

((1R,3R,6R)-6-methyl-3-(1-methylethenyl)cyclohexyl) 4-methylbenzenesulfonate, crude yield: $96 \%$

530

531

b) $2^{\text {nd }}$ intermediate:

532

S-((1S,3R,6R)-6-methyl-3-(1-methylethenyl)cyclohexyl) ethanethioate,

533 yield: $49 \%$,

534

MS-El: m/z (intensity in \%) 93 (100), 107 (98), 79 (91), 121 (74), 136 (65), 81 (37), 67

535

(34), 108 (27), 94 (25), 91 (24), 170 (22), 77 (20), 55 (19), 68 (17), 53 (16), 80 (15),

536

105 (12), 137 (11) 122 (9), 65 (8), 69 (8), 82 (7), 101 (5), 127 (5), $212\left(1, \mathrm{M}^{+}\right)$

537

538

c) analyte:

539

yield: $93 \%$

540

RI (FFAP): 1592

541

RI (DB-5): 1282

542

543

MS-El: m/z (intensity in \%) 79 (100), 121 (98), 107 (97), 93 (94), 136 (92), 41 (48), 81

544

(46), 67 (44), 68 (37), 55 (35), 91 (29), 95 (28), 53 (27), 94 (27), 77 (25), 108 (25),

545

$170\left(25, \mathrm{M}^{+}\right), 44$ (16), 80 (15), 92 (15), 69 (12), 105 (12), 122 (12), 137 (12), 82 (11),

546

65 (10), 119 (8), 155 (7)

547

548

MS-Cl (isobutane): $m / z$ (intensity in \%) $137(100), 171\left(28,[\mathrm{M}+\mathrm{H}]^{+}\right), 138(21), 136$ (13), 95 (11), 170 (6)

549

${ }^{1} \mathrm{H}$ NMR $\left(400 \mathrm{MHz} ; \mathrm{CDCl}_{3}\right): \delta 0.96(\mathrm{~d}, J=6.7 \mathrm{~Hz}, 3 \mathrm{H}, \mathrm{H}-\mathrm{C} 7), 1.14-1.21(\mathrm{~m}, 1 \mathrm{H}, \mathrm{H}-$

550

C5), 1.26 (d, J = 6.9 Hz, SH), $1.43-1.49$ (m, 2H, H-C6), 1.71 (s, 3H, H-C10), $1.69-$

1.79 (m, 3H, H-C1, H-C3, H-C5), $1.92-1.98$ (m, 1H, H-C3), 2.30 (tt, J=12.4, 3.3

$\mathrm{Hz}, 1 \mathrm{H}, \mathrm{H}-\mathrm{C} 4), 3.35-3.40$ (m, 1H, H-C2), 4.68 (d, J=1.0 Hz, 1H, H-C9), 4.70 (d, J=

$1.0 \mathrm{~Hz}, 1 \mathrm{H}, \mathrm{H}-\mathrm{C} 9)$

554

${ }^{13} \mathrm{C}$ NMR (101 MHz, $\mathrm{CDCl}_{3}$ ): $\delta 20.52$ (C7), 21.10 (C10), 28.14 (C6), 31.54 (C5),

35.97 (C1), 37.87 (C4), 40.17 (C3), 43.82 (C2), 108.61 (C9), 149.81 (C8) 


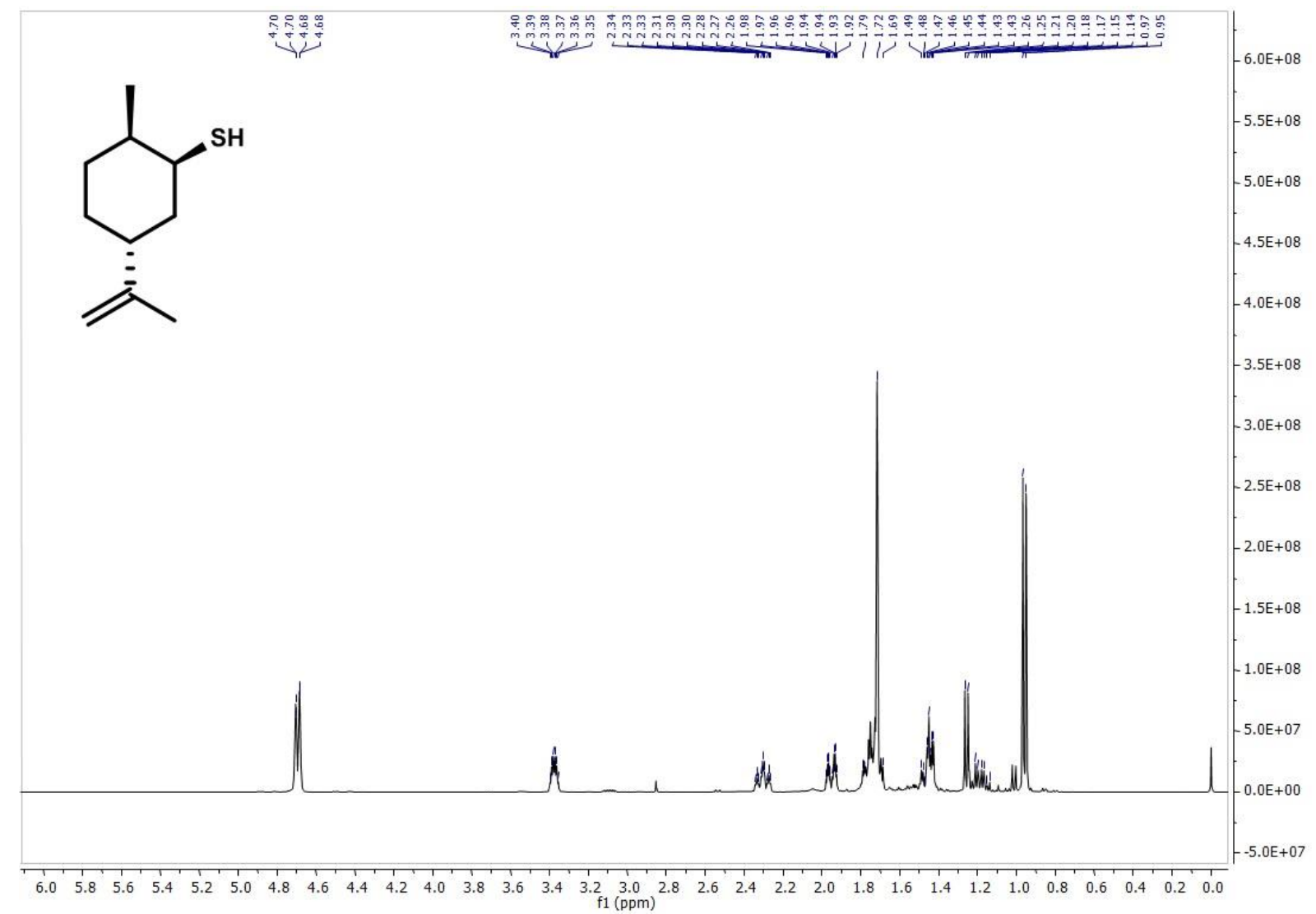

Figure S24. ${ }^{1} \mathrm{H}$ NMR spectrum of $(1 R, 2 S, 4 R)-8-p$-menthene-2-thiol.

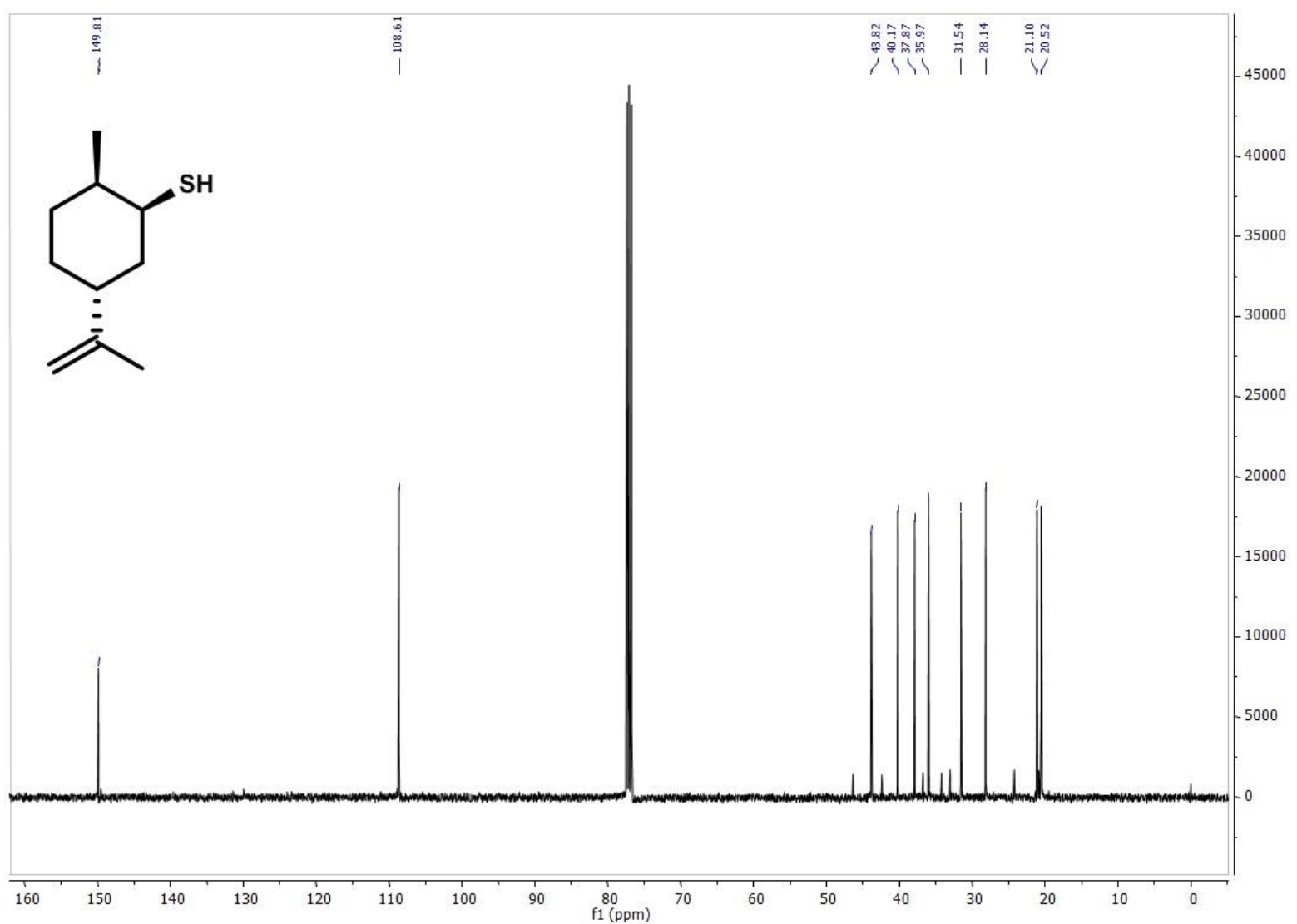

561 Figure S25. ${ }^{13} \mathrm{C}$ NMR spectrum of $(1 R, 2 S, 4 R)-8-p$-menthene-2-thiol. 
562

563

564

565

566

567

568

569

570

571

572

573

574

575

576

577

578

579

580

581

582

583

584

585

586

587

588

589

590

591

592

593

594

595

596

597

Compound 15b

\section{$(1 S, 2 R, 4 S)-8-p-m e n t h e n e-2-t h i o l$}

educt: (+)-dihydrocarveol

a) $1^{\text {st }}$ intermediate:

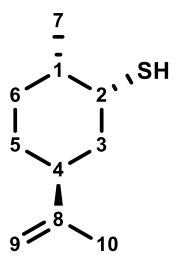

((1S,3S,6S)-6-methyl-3-(1-methylethenyl)cyclohexyl) 4-methylbenzenesulfonate, crude yield: $92 \%$

b) $2^{\text {nd }}$ intermediate:

S-((1R,3S,6S)-6-methyl-3-(1-methylethenyl)cyclohexyl) ethanethioate, yield: $65 \%$,

MS-El: m/z (intensity in \%) 93 (100), 107 (92), 79 (89), 121 (88), 136 (77), 67 (33), 81 (33), 108 (29), 91 (27), 170 (26), 94 (24), 55 (41), 95 (20), 77 (19), 92 (17), 68 (16), 53 (15), 155 (13), 105 (11), 137 (11), 122 (9), 69 (8), 82 (7), 101 (5), $212\left(1, \mathrm{M}^{+}\right)$

c) analyte:

yield: $75 \%$

RI (FFAP): 1591

RI (DB-5): 1282

MS-El: m/z (intensity in \%) 121 (100), 107 (98), 93 (95), 136 (94), 79 (93), 44 (52), 41 (48), 81 (42), 67 (38), 68 (34), 95 (30), 91 (29), 94 (27), 45 (25), 53 (25), 77 (25), 94 (25), 108 (22), 170 (22, M+), 137 (15), 69 (13), 65 (12), 80 (12), 105 (11), 122 (10), $155(9), 73(8)$

MS-Cl (isobutane): $m / z$ (intensity in \%) $137(100), 171\left(11,[\mathrm{M}+\mathrm{H}]^{+}\right), 138(9), 136(6)$, $95(6)$

${ }^{1} \mathrm{H}$ NMR $\left(400 \mathrm{MHz} ; \mathrm{CDCl}_{3}\right): \delta 0.96(\mathrm{~d}, J=6.6 \mathrm{~Hz}, 3 \mathrm{H}, \mathrm{H}-\mathrm{C} 7), 1.15-1.23(\mathrm{~m}, 1 \mathrm{H}, \mathrm{H}-$ C5), 1.26 (d, J=6.9 Hz, SH), $1.43-1.49$ (m, 2H, H-C6), $1.69-1.79$ (m, 3H, H-C1, H-C3, H-C5), 1.72 (s, 3H, H-C10), $1.92-1.98$ (m, 1H, H-C3), 2.30 (tt, J = 12.4, 3.3 $\mathrm{Hz}, 1 \mathrm{H}, \mathrm{H}-\mathrm{C} 4), 3.35-3.40$ (m, 1H, H-C2), 4.68 (d, J=1.1 Hz, 1H, H-C9), 4.70 (d, J= 1.1 Hz, 1H, H-C9)

${ }^{13} \mathrm{C}$ NMR (101 MHz, $\mathrm{CDCl}_{3}$ ): $\delta 20.52$ (C7), 21.10 (C10), 28.14 (C6), 31.54 (C5), 35.97 (C1), 37.87 (C4), 40.17 (C3), 43.82 (C2), 108.61 (C9), 149.81 (C8) 


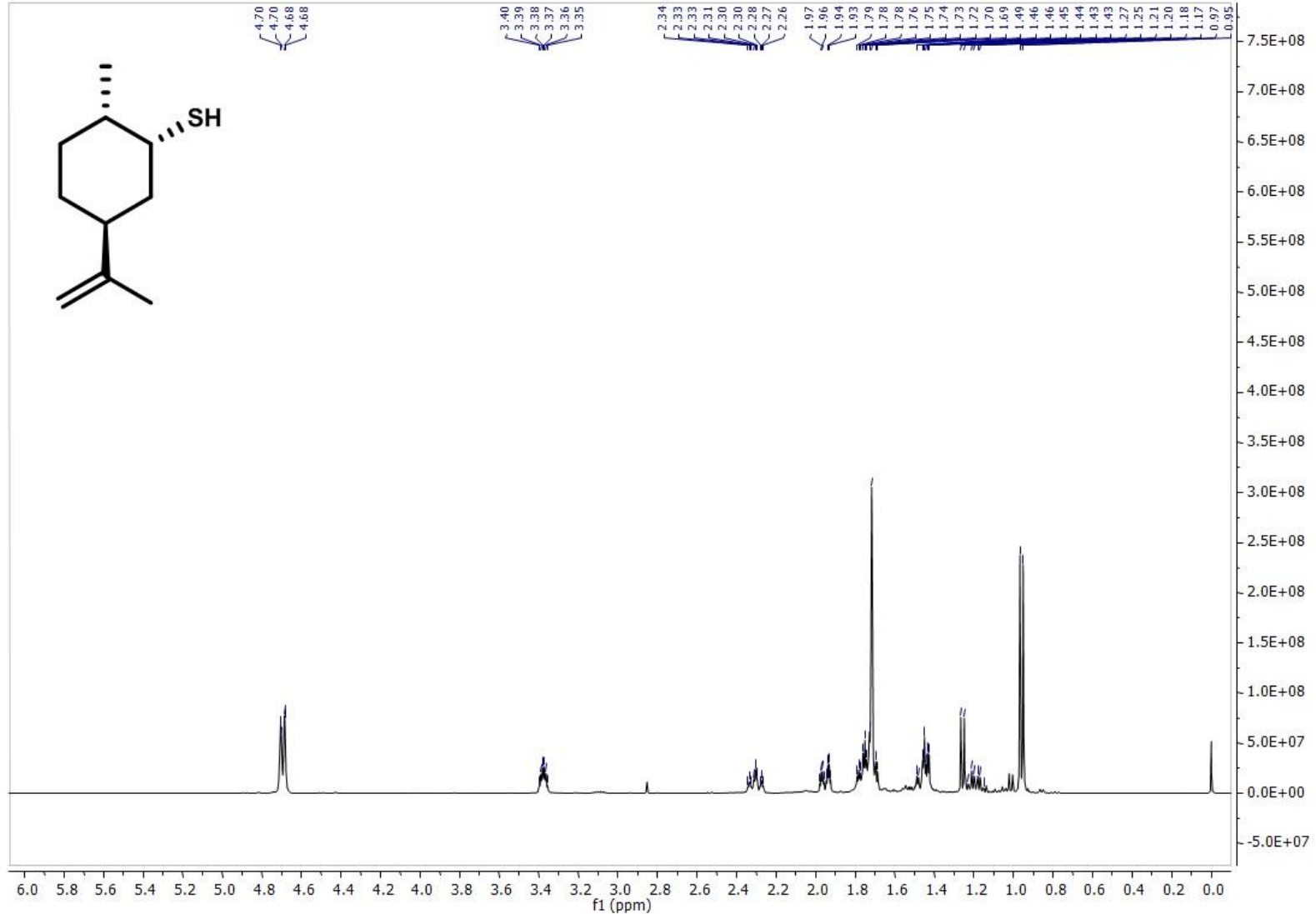

599 Figure S26. ${ }^{1} \mathrm{H}$ NMR spectrum of $(1 S, 2 R, 4 S)-8-p$-menthene-2-thiol.

600

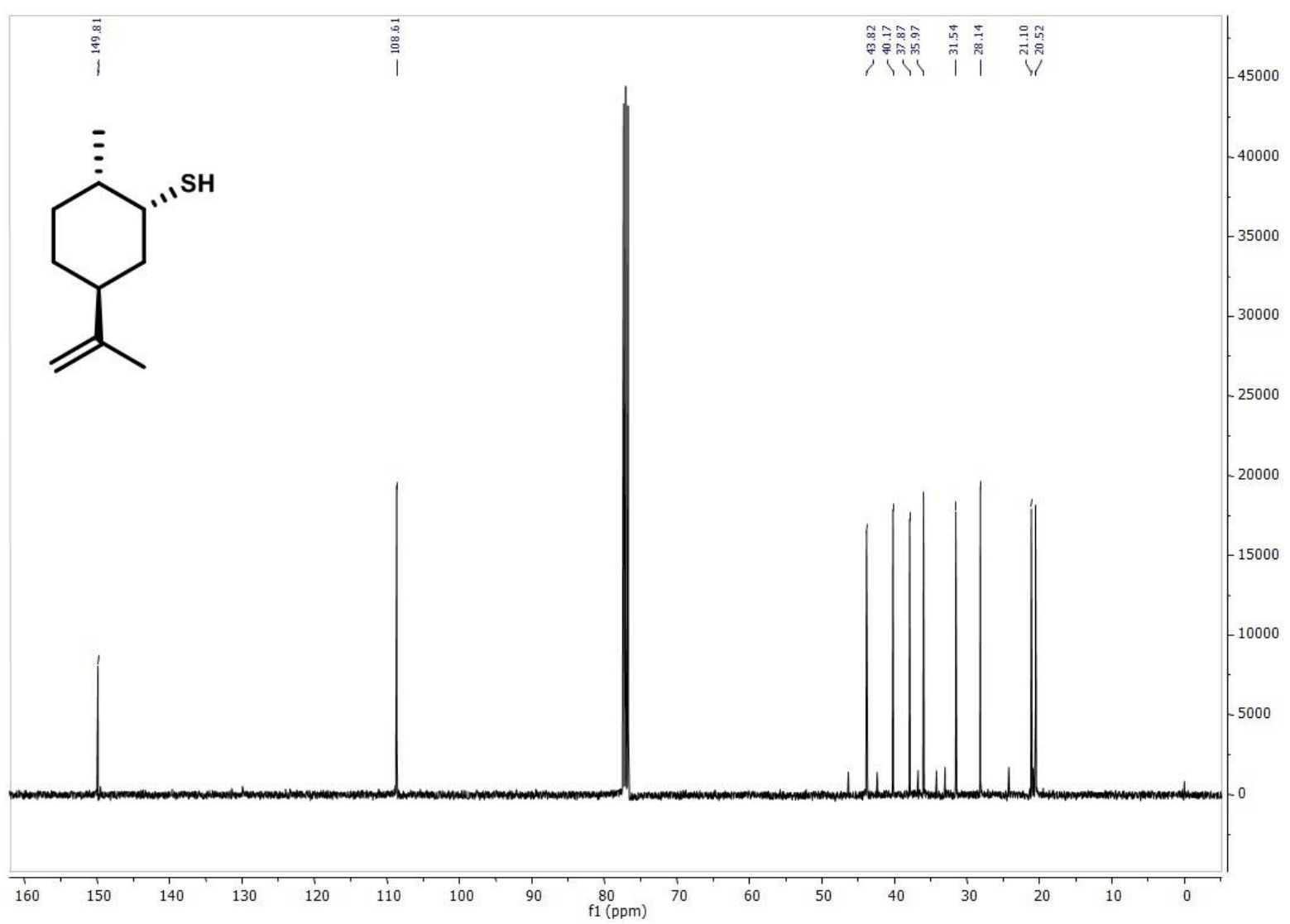

602 Figure S27. ${ }^{13} \mathrm{C}$ NMR spectrum of $(1 S, 2 R, 4 S)-8-p$-menthene-2-thiol. 
603

604

605

606

607

608

609

610

611

612

613

614

615

616

617

618

619

620

621

622

623

624

625

626

627

628

629

630

631

632

633

634

635

636
Compound 16a

\section{$(1 R, 3 S, 4 S)-8-p$-menthene-3-thiol}

educt: (-)-isopulegol

a) $1^{\text {st }}$ intermediate:

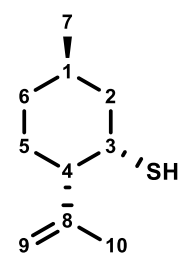

((1R,2S,5R)-5-methyl-2-(1-methylethenyl)cyclohexyl) 4-methylbenzenesulfonate, crude yield: $86 \%$

b) $2^{\text {nd }}$ intermediate:

S-((1S,2S,5R)-5-methyl-2-(1-methylethenyl)cyclohexyl) ethanethioate, yield: $65 \%$,

MS-El: m/z (intensity in \%) 81 (100), 155 (98), 95 (57), 94 (52), 79 (49), 67 (46), 121 (29), 55 (28), 107 (23), 123 (22), 170 (22), 99 (21), 75 (19), 68 (19), 69 (17), 53 (16), 97 (16), 85 (13), 78 (11), 109 (10), $113(8), 212\left(1, \mathrm{M}^{+}\right)$

c) analyte:

yield: $67 \%$

RI (FFAP): 1558

RI (DB-5): 1266

MS-El: m/z (intensity in \%) 155 (100), 81 (52), 95 (36), $170\left(35, \mathrm{M}^{+}\right), 121$ (31), 93 (28), 79 (25), 137 (24), 136 (22), 123 (21), 67 (19), 107 (19), 111 (19), 55 (15), 87 (14), 91 (14), 99 (13), 156 (12), 168 (12), 68 (11), 77 (10), 101 (10), 69 (8), 109 (7), $113(7), 127(7), 153(5), 171$ (5)

MS-Cl (isobutane): $\mathrm{m} / z$ (intensity in \%) $171\left(100,[\mathrm{M}+\mathrm{H}]^{+}\right), 137(31), 172(11), 173(7)$ ${ }^{1} \mathrm{H}$ NMR (400 MHz; $\left.\mathrm{CDCl}_{3}\right): \delta 0.89-1.02(\mathrm{~m}, 2 \mathrm{H}, \mathrm{H}-\mathrm{C} 2, \mathrm{H}-\mathrm{C} 6), 0.95$ (d, J = $6.6 \mathrm{~Hz}$, $3 \mathrm{H}, \mathrm{H}-\mathrm{C} 7), 1.27-1.38$ (m, 1H, H-C5), $1.45-1.54(\mathrm{~m}, 1 \mathrm{H}, \mathrm{H}-\mathrm{C} 1), 1.64-1.70(\mathrm{~m}, 2 \mathrm{H}$, $\mathrm{H}-\mathrm{C} 5, \mathrm{H}-\mathrm{C} 6), 1.71$ (s, 3H, H-C10), $1.85-1.92(\mathrm{~m}, 2 \mathrm{H}, \mathrm{H}-\mathrm{C} 4, \mathrm{SH}), 2.01-2.07$ (m, $1 \mathrm{H}, \mathrm{H}-\mathrm{C} 2$ ), 3.46 (ddd, $J=10.8,10.0,4.3 \mathrm{~Hz}, 1 \mathrm{H}, \mathrm{H}-\mathrm{C} 3$ ), $4.85-4.86$ (m, $1 \mathrm{H}, \mathrm{H}-\mathrm{C} 9$ ), $4.89-4.91(\mathrm{~m}, 1 \mathrm{H}, \mathrm{H}-\mathrm{C} 9)$

${ }^{13} \mathrm{C} \mathrm{NMR}\left(101 \mathrm{MHz}, \mathrm{CDCl}_{3}\right): \delta 19.20$ (C10), 22.23 (C7), 29.66 (C5), 31.45 (C1), 34.33 (C6), 42.68 (C2), 54.14 (C4), 70.36 (C3), 112.83 (C9), 146.65 (C8) 


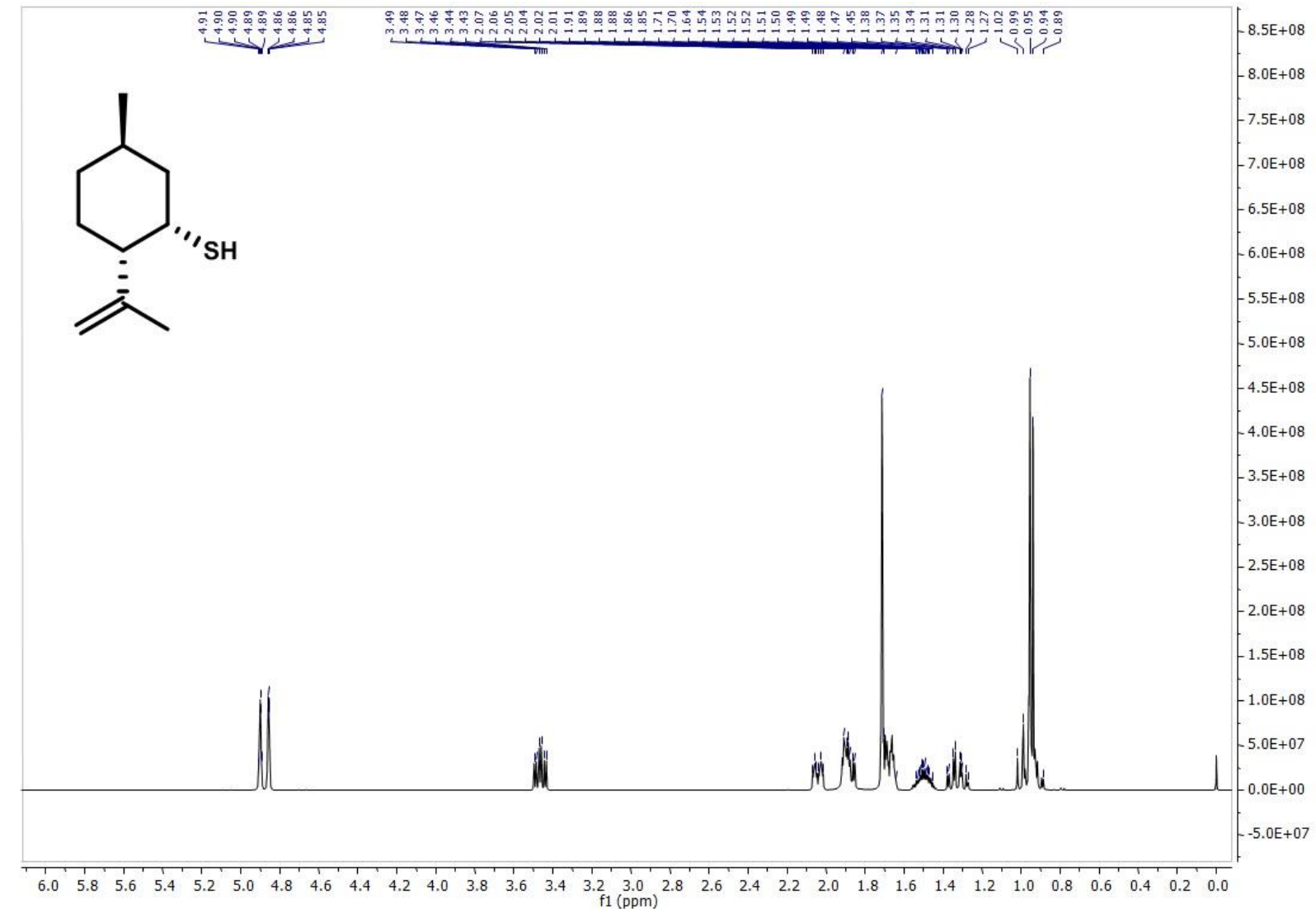

Figure S28. ${ }^{1} \mathrm{H}$ NMR spectrum of $(1 R, 3 S, 4 S)-8-p$-menthene-3-thiol.

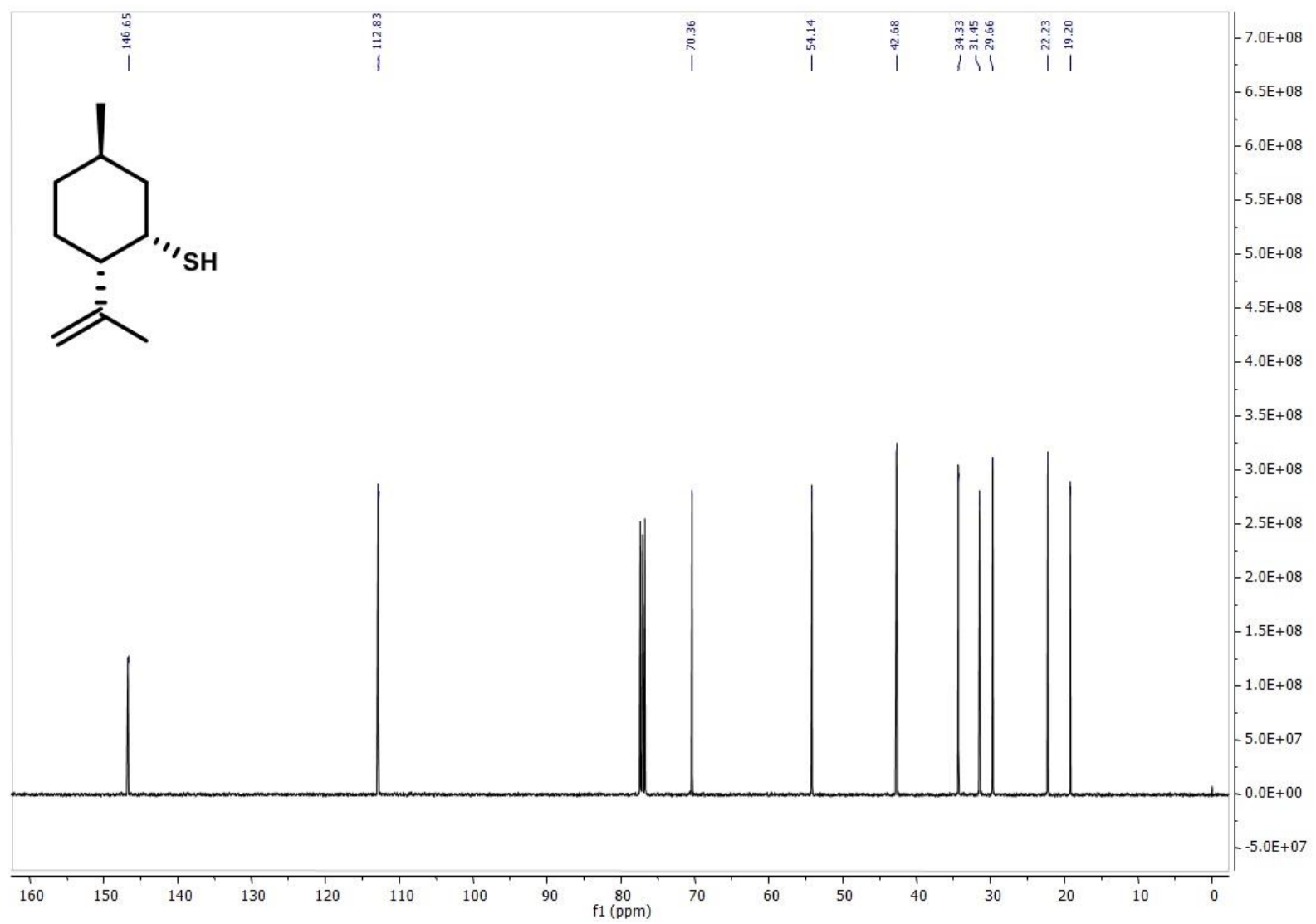

641 Figure S29. ${ }^{13} \mathrm{C}$ NMR spectrum of $(1 R, 3 S, 4 S)-8-p$-menthene-3-thiol. 
642 Compound 16b

643

\begin{tabular}{|c|c|}
\hline 644 & $(1 S, 3 R, 4 R)-8-p$-menthene-3-thiol \\
\hline 645 & \\
\hline 646 & educt: (+)-isopulegol \\
\hline 647 & \\
\hline 648 & a) $1^{\text {st }}$ intermediate: \\
\hline 649 & $((1 S, 2 R, 5 S)-5-m e t h y l-2-(1-m e t h y l e t h e n y l) c y c l o h e x y l)$ 4-methylbenzenesulfonate, \\
\hline 650 & crude yield: $79 \%$ \\
\hline 51 & \\
\hline 652 & b) $2^{\text {nd }}$ intermediate: \\
\hline 653 & S-((1R,2R,5S)-5-methyl-2-(1-methylethenyl)cyclohexyl) ethanethioate, \\
\hline 654 & yield: $84 \%$ \\
\hline 655 & MS-El: m/z (intensity in \%) 128 (100), 169 (48), 95 (46), 93 (45), 81 (42), 107 (28), 43 \\
\hline 656 & (27), 79 (27), 135 (25), 136 (22), 67 (20), 121 (17), 55 (16), 113 (15), 41 (13), 94 (13), \\
\hline 657 & 155 (13), 79 (12), $91(12), 108$ (9), 170 (9), 53 (8), 69 (8), 85 (8), 99 (8), 129 (8), 164 \\
\hline 658 & $(7), 212\left(3, \mathrm{M}^{+}\right)$ \\
\hline
\end{tabular}




\begin{tabular}{|c|c|}
\hline 659 & c) analyte: \\
\hline 660 & yield: $62 \%$ \\
\hline 661 & RI (FFAP): 1558 \\
\hline 662 & RI (DB-5): 1267 \\
\hline 63 & \\
\hline 664 & MS-El: $m / z$ (intensity in \%) 81 (100), 155 (75), 95 (58), 41 (51), 93 (48), 79 (47), 67 \\
\hline 665 & (46), $55(44), 121(38), 68(29), 123(27), 107(26), 53(25), 170\left(25, M^{+}\right), 77(23), 137$ \\
\hline 666 & (23), 69 (22), 91 (21), 99 (21), 87 (20), $111(20), 136$ (20), 101 (16), 75 (15), 94 (15), \\
\hline 667 & 109 (15), 77 (14), 85 (14), 82 (12), 80 (12), 127 (11), 113 (10), 156 (9), 168 (9), 92 \\
\hline 668 & $(8), 105(8), 171(3)$ \\
\hline 669 & MS-Cl (isobutane): $m / z$ (intensity in \%) $171\left(100,[\mathrm{M}+\mathrm{H}]^{+}\right), 137$ (29), 95 (14), 91 (10), \\
\hline 670 & $172(9), 155(5)$ \\
\hline 671 & ${ }^{1} \mathrm{H}$ NMR $\left(400 \mathrm{MHz} ; \mathrm{CDCl}_{3}\right): \delta 0.89-1.02(\mathrm{~m}, 2 \mathrm{H}, \mathrm{H}-\mathrm{C} 2, \mathrm{H}-\mathrm{C} 6), 0.95(\mathrm{~d}, J=6.6 \mathrm{~Hz}$ \\
\hline 672 & $3 \mathrm{H}, \mathrm{H}-\mathrm{C} 7), 1.28-1.38(\mathrm{~m}, 1 \mathrm{H}, \mathrm{H}-\mathrm{C} 5), 1.45-1.54(\mathrm{~m}, 1 \mathrm{H}, \mathrm{H}-\mathrm{C} 1), 1.65-1.70(\mathrm{~m}, 2 \mathrm{H}$, \\
\hline 673 & $\mathrm{H}-\mathrm{C} 5, \mathrm{H}-\mathrm{C} 6), 1.71(\mathrm{~s}, 3 \mathrm{H}, \mathrm{H}-\mathrm{C} 10), 1.85-1.92(\mathrm{~m}, 2 \mathrm{H}, \mathrm{H}-\mathrm{C} 4, \mathrm{SH}), 2.01-2.07(\mathrm{~m}$, \\
\hline 674 & 1H, H-C2), 3.46 (ddd, J=10.8, 10.0, 4.3 Hz, 1H, H-C3), $4.85-4.86$ (m, 1H, H-C9), \\
\hline 675 & $4.89-4.91(\mathrm{~m}, 1 \mathrm{H}, \mathrm{H}-\mathrm{C} 9)$ \\
\hline 676 & ${ }^{13} \mathrm{C}$ NMR (101 MHz, $\left.\mathrm{CDCl}_{3}\right): \delta 19.20$ (C10), 22.23 (C7), 29.66 (C5), 31.45 (C1), \\
\hline 677 & 34.33 (C6), 42.68 (C2), 54.14 (C4), 70.36 (C3), 112.83 (C9), 146.65 (C8) \\
\hline
\end{tabular}




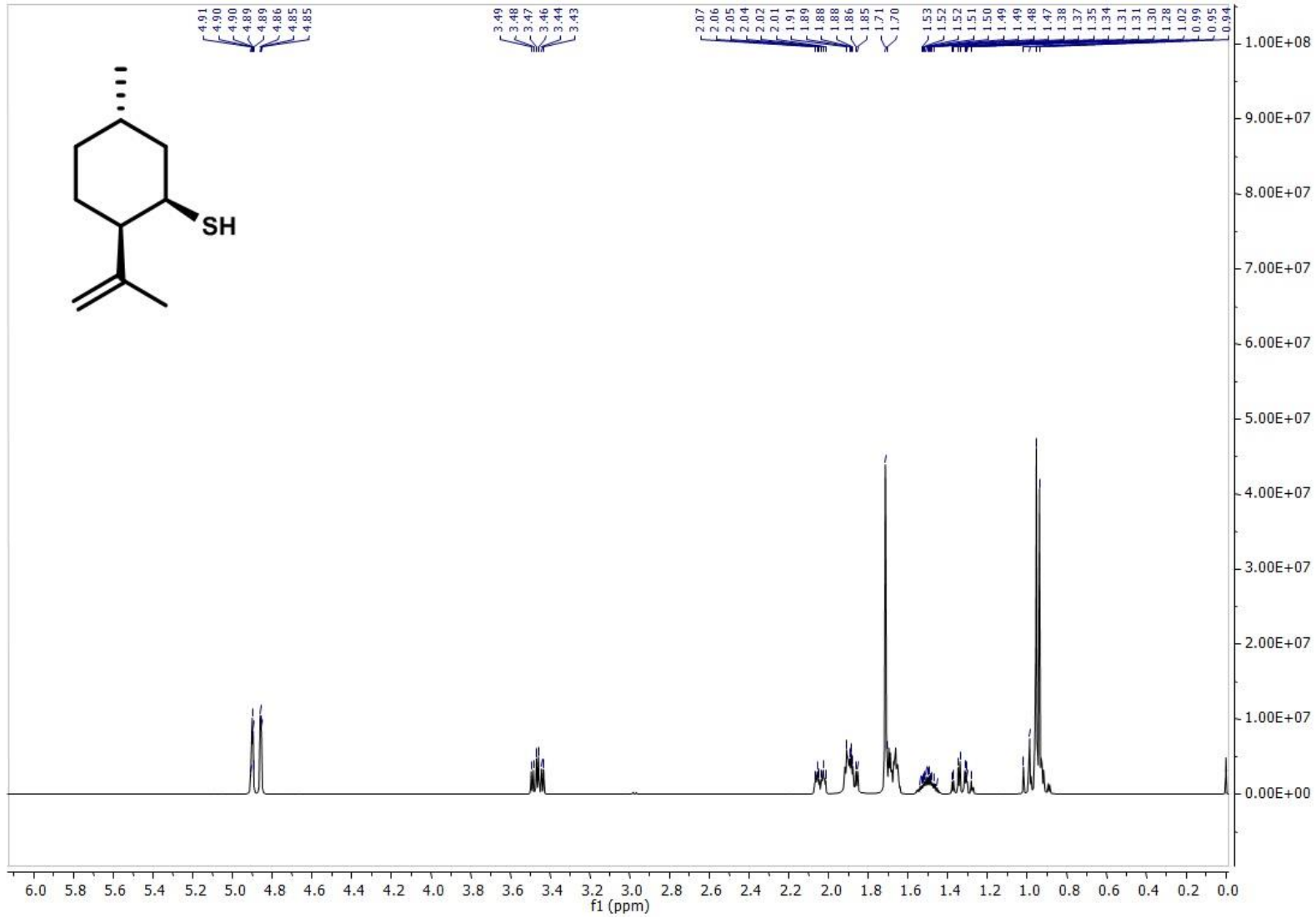

679 Figure S30. ${ }^{1} \mathrm{H}$ NMR spectrum of $(1 S, 3 R, 4 R)-8-p$-menthene-3-thiol.

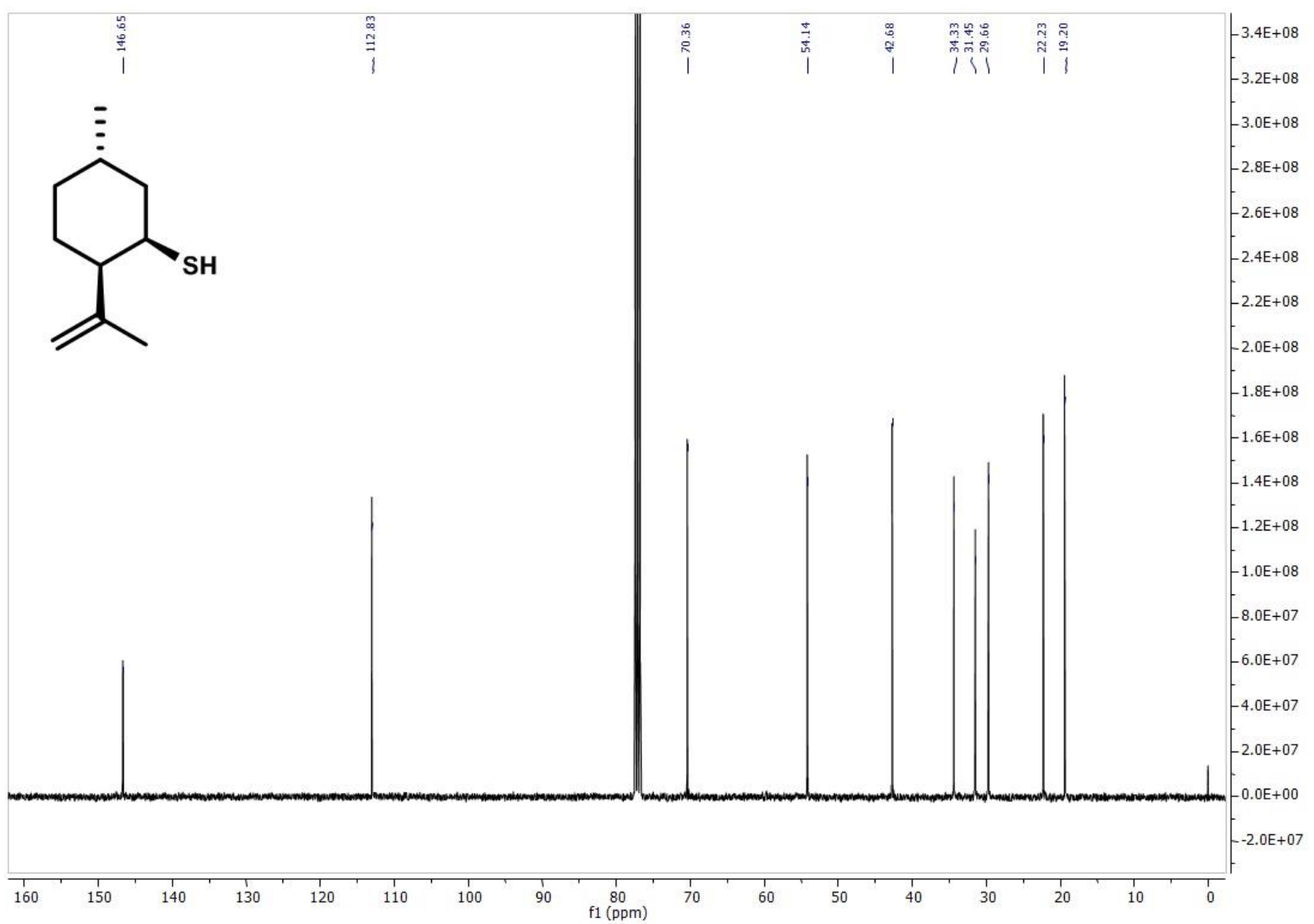

682 Figure S31. ${ }^{13} \mathrm{C}$ NMR spectrum of $(1 S, 3 R, 4 R)-8-p$-menthene-3-thiol. 
683 Compound 18a

684

685

(3R)- $\beta$-thio citronellol

686

687

educt: $(R)-(+)-\beta$-citronellol

688

689

a) $1^{\text {st }}$ intermediate:

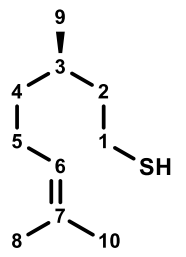

690

(3R)-3,7-dimethyloct-6-en-1-yl 4-methylbenzenesulfonate,

691

crude yield: $62 \%$

692

693

b) $2^{\text {nd }}$ intermediate:

694

695

(3R)-S-(3,7-dimethyloct-6-en-1-yl) ethanethioate,

yield: $93 \%$,

696

MS-El: m/z (intensity in \%) 69 (100), 171 (58), 115 (49), 55 (31), 81 (28), 95 (22), 101

697

(20), 67 (19), 82 (14), 68 (9), 123 (8), 83 (8), 138 (7), 53 (6), 103 (6), 47 (6), 137 (6),

698

$172(6), 214\left(2, \mathrm{M}^{+}\right)$

699

700

c) analyte:

701

yield: $76 \%$

702

RI (FFAP): 1541

703

RI (DB-5): 1278

704

705

MS-El: m/z (intensity in \%) 69 (100), 55 (51), 115 (45), 67 (33), 81 (31), 101 (29), 95

706

(29), 129 (27), 82 (20), 47 (16), 68 (15), 83 (14), 56 (12), 61 (12), 123 (12), 70 (11),

707

$53(10), 171(10), 172\left(8, \mathrm{M}^{+}\right)$

708

MS-Cl (isobutane): $m / z$ (intensity in \%) $173\left(100,[\mathrm{M}+\mathrm{H}]^{+}\right), 174(14), 139$ (8)

709

${ }^{1} \mathrm{H}$ NMR $\left(400 \mathrm{MHz} ; \mathrm{CDCl}_{3}\right): \delta 0.91(\mathrm{~d}, J=6.5 \mathrm{~Hz}, 3 \mathrm{H}, \mathrm{H}-\mathrm{C} 9), 1.13-1.22(\mathrm{~m}, 1 \mathrm{H}, \mathrm{H}-$

710

C4), $1.30-1.39$ (m, 1H, H-C4), 1.33 (t, J = 7.6 Hz, SH), $1.43-1.52$ (m, 1H, H-C2),

711

1.53 - 1.69 (m, 2H, H-C2, H-C3), 1.63 (s, 3H, H-C10), 1.71 (s, 3H, H-C8), 1.92 -

712

2.08 (m, 2H, H-C5), $2.48-2.64$ (m, 2H, H-C1), $5.09-5.14$ (m, 1H, H-C6)

713

${ }^{13} \mathrm{C}$ NMR (101 MHz, CDCl 3 ): $\delta 17.66$ (C3), 19.12 (C9), 22.48 (C1), 25.41 (C5), 25.72

714

(C10), 31.49 (C8), 36.72 (C4), 41.44 (C2), 124.63 (C6), 131.34 (C7) 


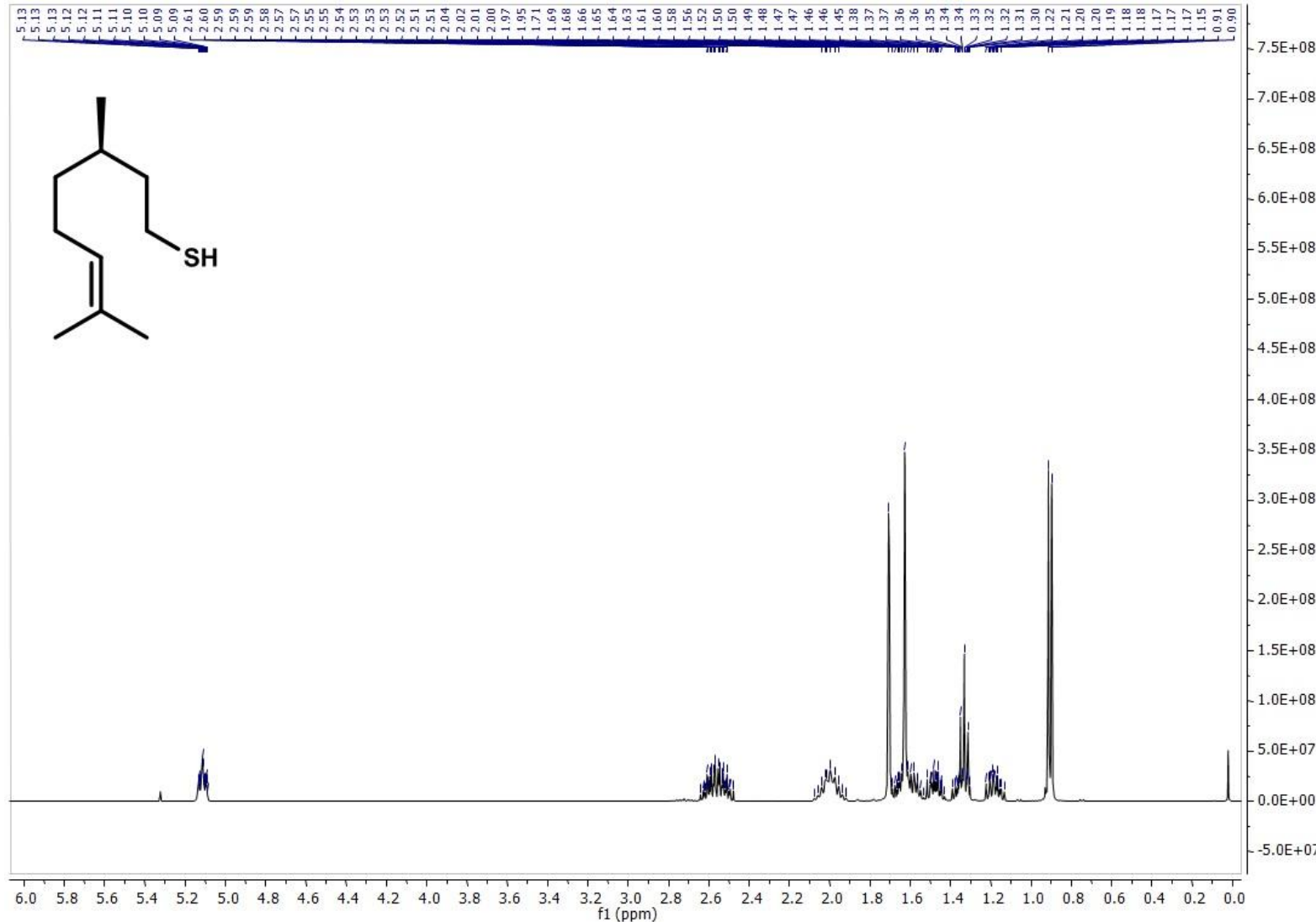

717 Figure S32. ${ }^{1} \mathrm{H}$ NMR spectrum of $(3 R)-\beta$-thio citronellol.

718

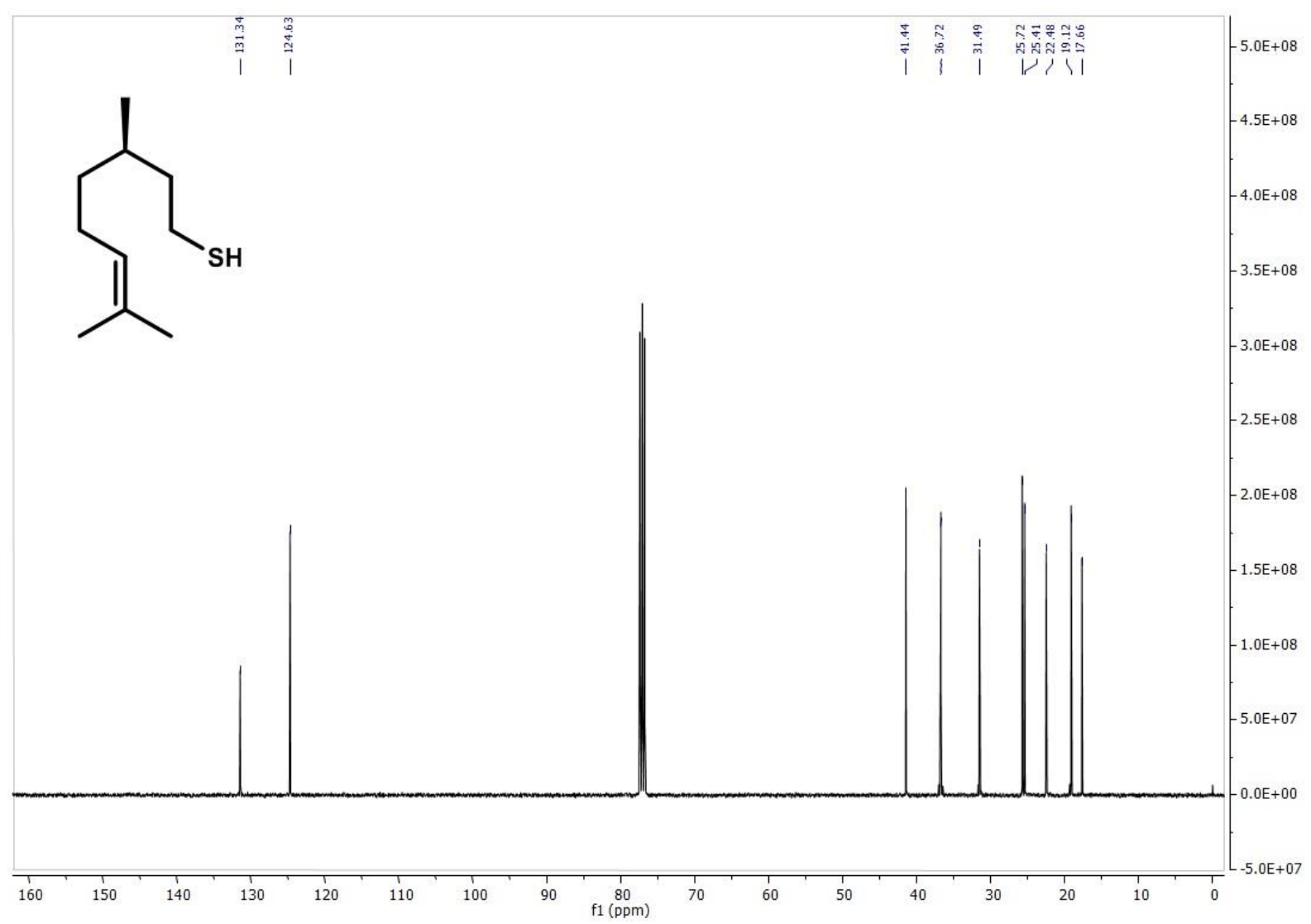

Figure S33. ${ }^{13} \mathrm{C}$ NMR spectrum of $(3 R)-\beta$-thio citronellol. 


\section{Compound 18b}

722

723

(3S)- $\beta$-thio citronellol

724

725

educt: (S)-(-)- $\beta$-citronellol

726

727

a) $1^{\text {st }}$ intermediate:

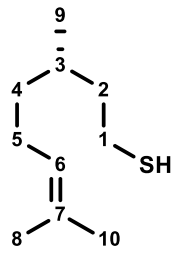

728

729

(3S)-3,7-dimethyloct-6-en-1-yl 4-methylbenzenesulfonate,

crude yield: $51 \%$

730

731

b) $2^{\text {nd }}$ intermediate:

732

(3S)-S-(3,7-dimethyloct-6-en-1-yl) ethanethioate,

733 yield: $87 \%$,

734

MS-El: $m / z$ (intensity in \%) 69 (100), 171 (69), 115 (56), 81 (30), 55 (28), 95 (24), 101

735

(23), 68 (18), 82 (15), 172 (11), 123 (10), 138 (9), 83 (8), 137 (8), $172(5), 214\left(3, \mathrm{M}^{+}\right)$

736

737

c) analyte:

738

yield: $84 \%$

739

RI (FFAP): 1541

740

RI (DB-5): 1278

741

742

MS-El: $m / z$ (intensity in \%) 69 (100), 55 (68), 115 (58), 67 (36), 81 (34), 101 (34), 129

743

744

(30), 95 (27), 47 (20), 82 (20), 68 (14), 83 (14), 56 (13), 61 (13), 123 (12), 53 (11), 70 (11), 87 (10), $171(10), 57(8), 172\left(8, \mathrm{M}^{+}\right)$

745

MS-Cl (isobutane): $m / z$ (intensity in \%) $173\left(100,[\mathrm{M}+\mathrm{H}]^{+}\right), 174(12)$

746

747

748

749

750

751

${ }^{1} \mathrm{H} \mathrm{NMR}\left(400 \mathrm{MHz} ; \mathrm{CDCl}_{3}\right): \delta 0.88$ (d, 3H, H-C9), $1.14-1.20$ (m, $\left.1 \mathrm{H}, \mathrm{H}-\mathrm{C} 4\right), 1.28$ 1.35 (m, 1H, H-C4), 1.31 (t, J=7.6 Hz, SH), $1.42-1.49$ (m, 1H, H-C2), $1.52-1.65$ (m, 1H, H-C3), 1.60 (s, 3H, H-C10), $1.53-1.65$ (m, 1H, H-C2), 1.69 (s, 3H, H-C8), $1.90-2.05$ (m, 2H, H-C5), 2.45 - 2.62 (m, 2H, H-C1), $5.07-5.11$ (m, 1H, H-C6) ${ }^{13} \mathrm{C} \mathrm{NMR}\left(101 \mathrm{MHz}, \mathrm{CDCl}_{3}\right): \delta 17.66$ (C3), 19.12 (C9), 22.48 (C1), 25.41 (C5), 25.72 (C10), 31.49 (C8), 36.72 (C4), 41.44 (C2), 124.63 (C6), 131.34 (C7) 


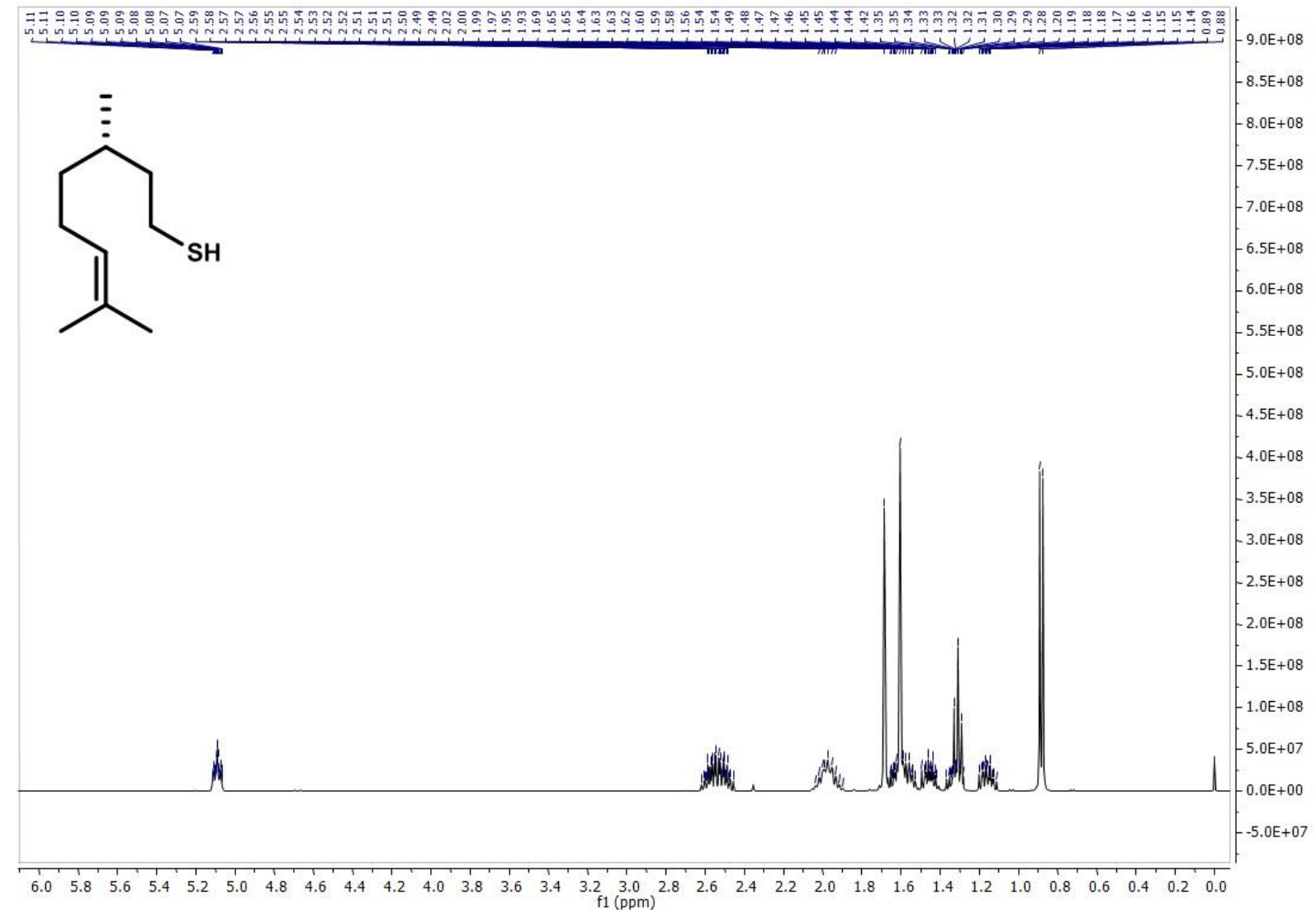

753 Figure S34. ${ }^{1} \mathrm{H}$ NMR spectrum of $(3 S)-\beta$-thio citronellol.

754

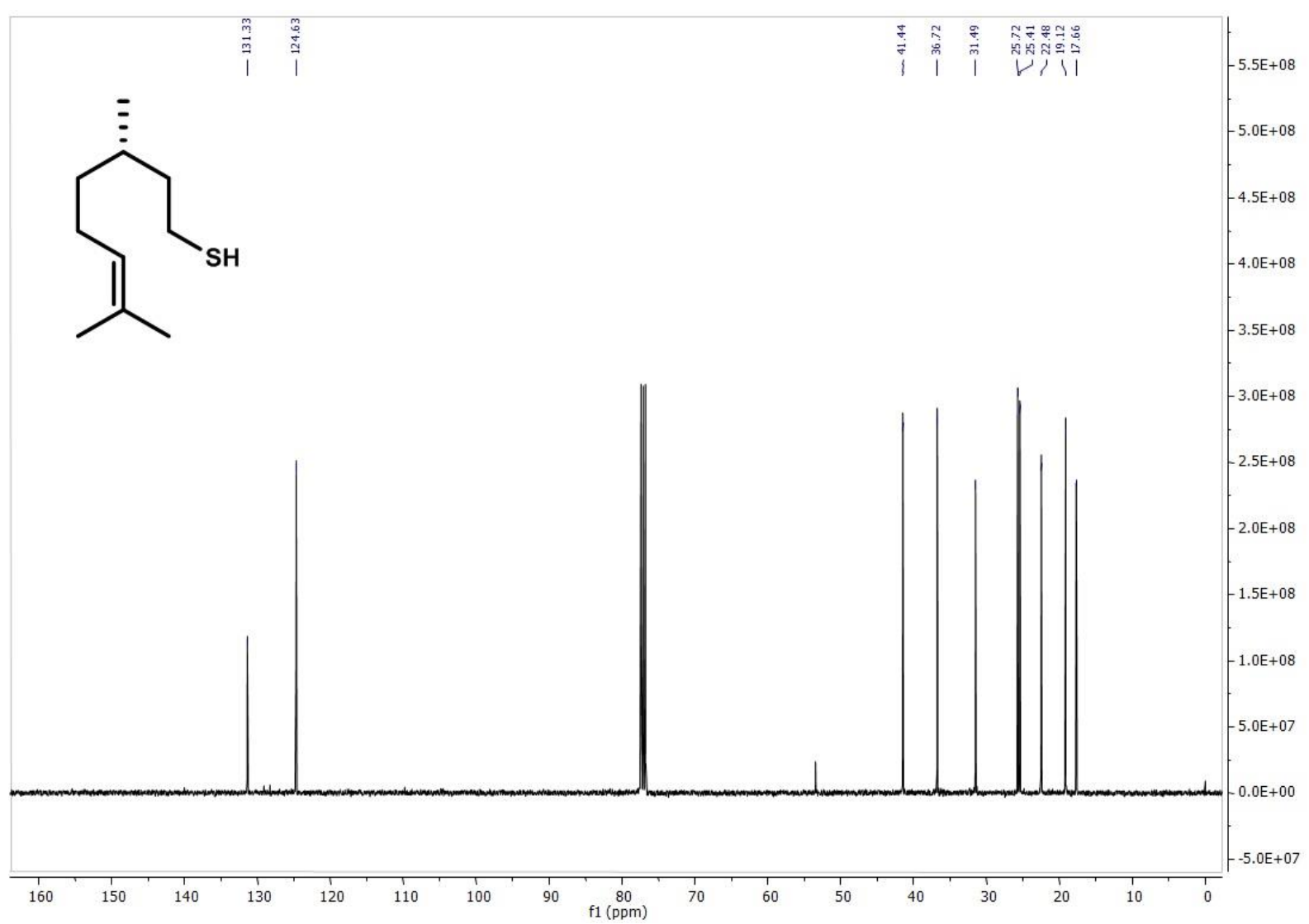

756 Figure S35. ${ }^{13} \mathrm{C}$ NMR spectrum of (3S)- $\beta$-thio citronellol. 
757 Compound 22

758

759

\section{dihydro thio citronellol}

760

761

educt: dihydro citronellol

762

763

a) $1^{\text {st }}$ intermediate:

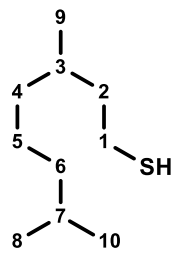

764

3,7-dimethyloctyl 4-methylbenzenesulfonate,

765

crude yield: $72 \%$

766

767

b) $2^{\text {nd }}$ intermediate:

768

S-(3,7-dimethyloctyl) ethanethioate,

769

yield: $79 \%$,

770

MS-El: m/z (intensity in \%) 43 (100), 156 (49), 70 (48), 83 (43), 173 (40), 69 (38), 55

771

(32), 57 (24), 56 (21), 111 (18), 71 (17), 84 (14), 97 (12), 140 (12), 85 (11), 112 (7),

772

$125(6), 132(5), 216\left(1, M^{+}\right)$

773

774

c) analyte:

775

yield: $87 \%$

776

RI (FFAP): 1440

777

RI (DB-5): 1242

778

779

MS-El: m/z (intensity in \%) 70 (100), 56 (58), 69 (53), 55 (47), 57 (26), 84 (26), 72

780

(25), 140 (25), 111 (22), 83 (19), 51 (12), 47 (11), 97 (9), 112 (9), 85 (7), 125 (6), 67

781

(5), $141(4), 174\left(2, \mathrm{M}^{+}\right)$

782

MS-Cl (isobutane): $m / z$ (intensity in \%) $175\left(100,[\mathrm{M}+\mathrm{H}]^{+}\right), 141$ (19), $140(17), 176$

783

(13), $173(11)$

784

785

${ }^{1} \mathrm{H}$ NMR (400 MHz; $\mathrm{CDCl}_{3}$ ): $\delta 0.87$ (d, J = 6.7 Hz, 9H, H-C8, H-C9, H-C10), 1.09 1.17 (m, 3H, H-C4, H-C5), 1.22 - 1.28 (m, 3H, H-C4, H-C6), 1.31 (t, J = 7.5 Hz, SH),

786

$1.41-1.63$ (m, 4H, H-C2, H-C3, H-C7), 2.49 - 2.58 (m, 2H, H-C1)

787

${ }^{13} \mathrm{C}$ NMR (101 MHz, $\mathrm{CDCl}_{3}$ ): $\delta 19.22$ (C9), 22.53 (C1), 22.60 (C8), 22.70 (C10),

788 


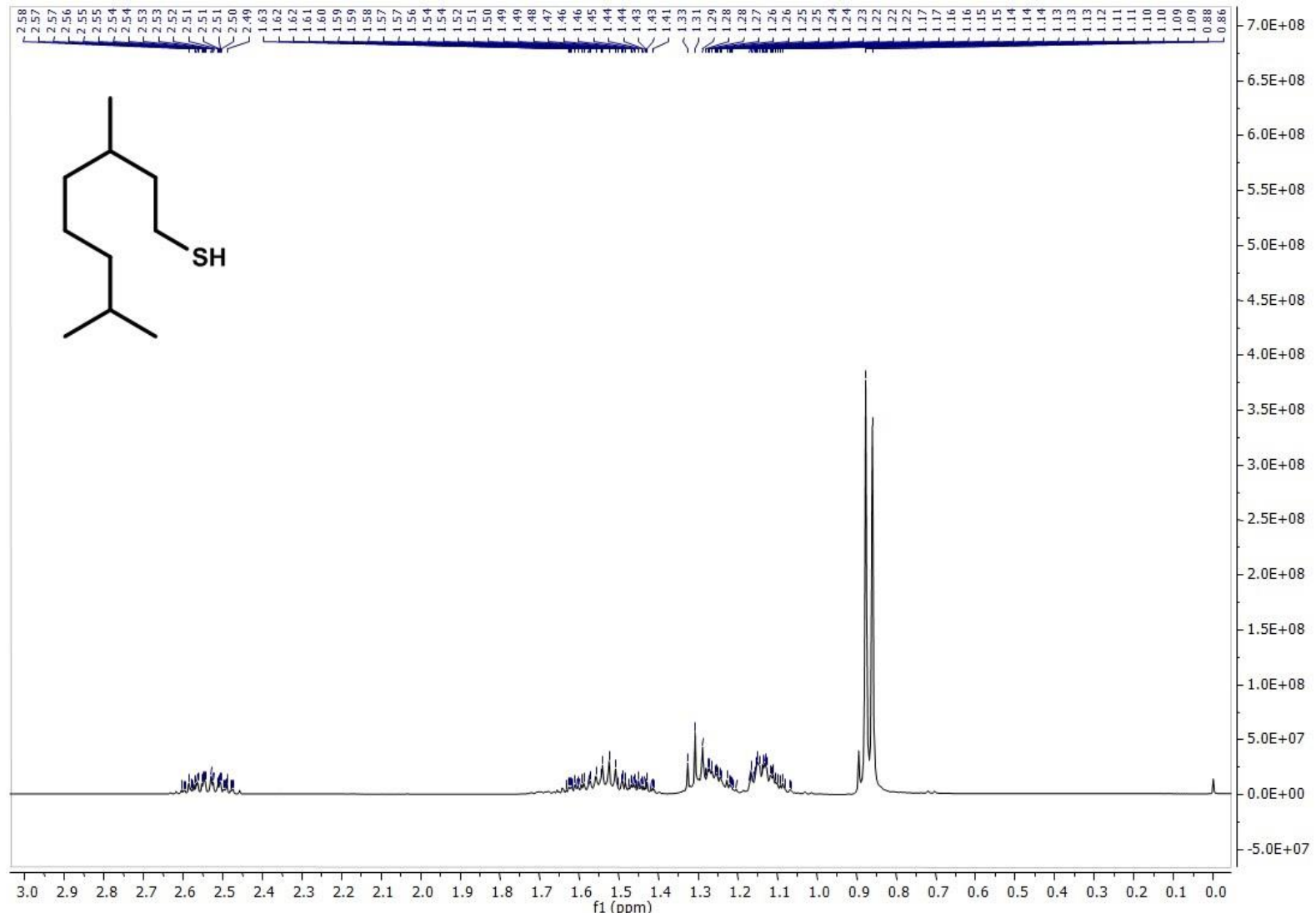

790 Figure S36. ${ }^{1} \mathrm{H}$ NMR spectrum of dihydro thio citronellol.

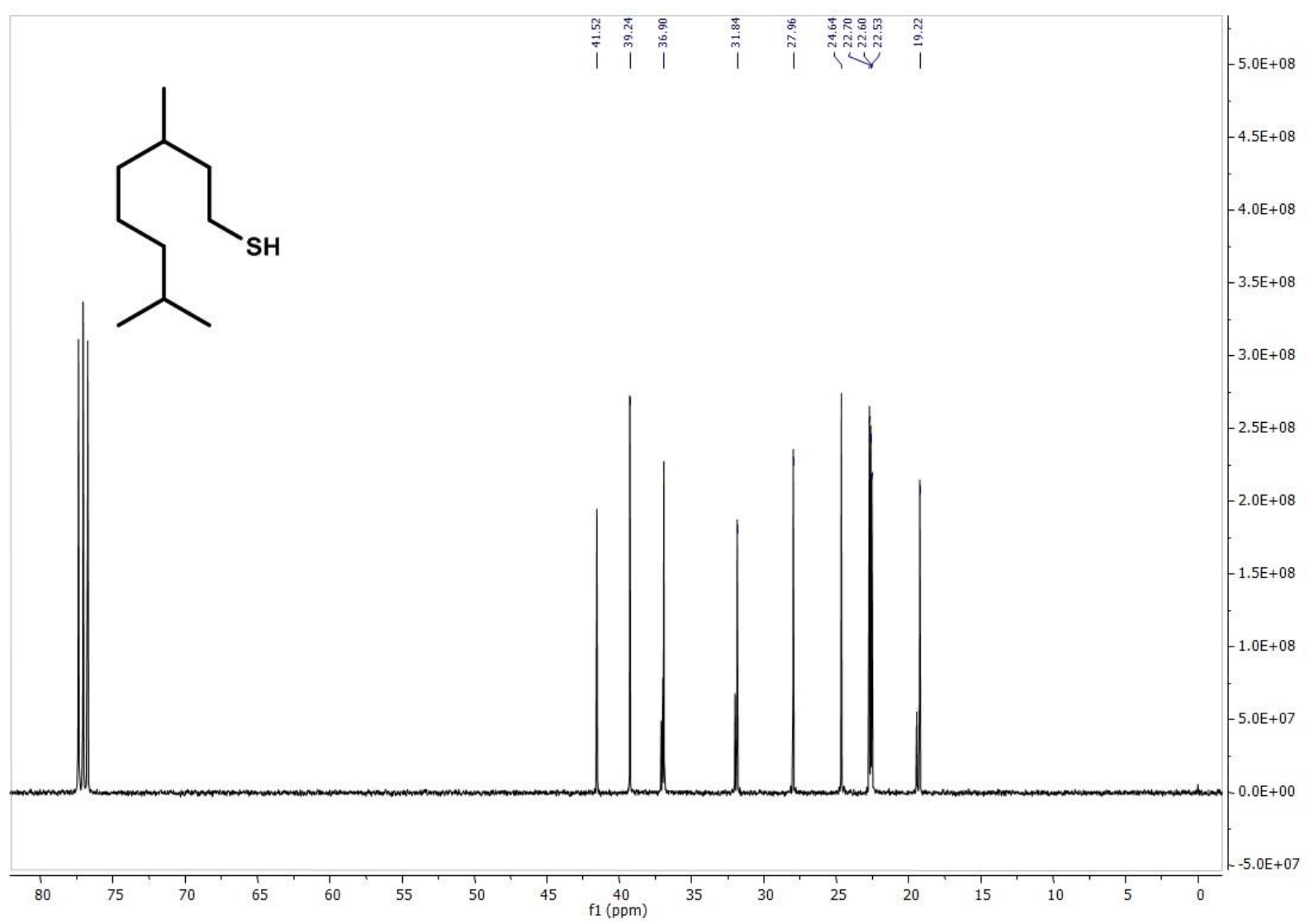

793 Figure S37. ${ }^{13} \mathrm{C}$ NMR spectrum of dihydro thio citronellol. 
794

795

796

797

798

799

800

801

802

803

804

805

806

807

808

809

810

811

812

813

814

815

816

817

818

819

820

821

822

823

\section{Synthetic route B for mercaptan preparation}

Eight compounds were obtained by using a strategy consisting of direct tosylation and reduction as shown in Figure $\mathbf{S} 38$.

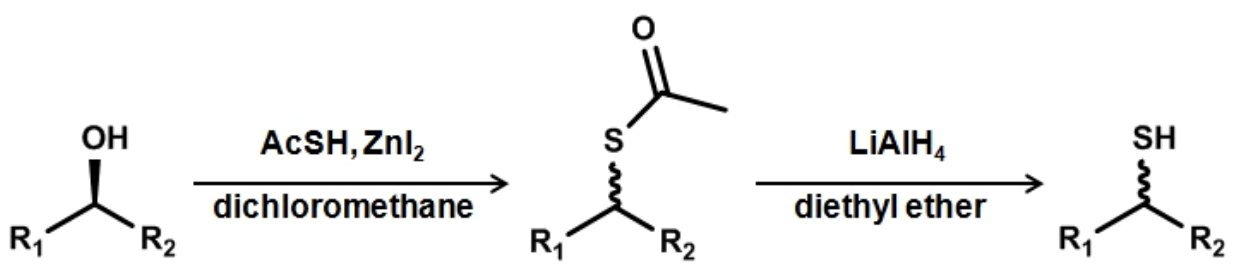

$R_{1}, R_{2}=H$, alkyl

Figure S38. Route assigned as "synthetic route B".

a) Direct thioacetylation. The alcohol $(10 \mathrm{mmol})$ was dissolved in dichloromethane $(20 \mathrm{~mL})$ and a mixture of thioacetic acid $(0.92 \mathrm{~g} ; 12 \mathrm{mmol})$ and zinc iodide $(1.60 \mathrm{~g} ; 5 \mathrm{mmol}$ ) was added. The reaction mixture was stirred at RT for $5 \mathrm{~h}$, then diluted with water $(20 \mathrm{~mL})$ and filtered. The residue was washed with dichloromethane $(15 \mathrm{~mL})$ and the aqueous layer was also washed twice with dichloromethane $(20 \mathrm{~mL})$. The combined organic layers were washed with saturated sodium hydrogen carbonate solution $(20 \mathrm{~mL})$ and brine $(20 \mathrm{~mL})$ and were dried over sodium sulfate. The solvent was removed under reduced pressure and the thioacetate was purified by column chromatography on silica gel with pentane/diethyl ether (98+2; by vol.) as the eluent.

b) Reduction to the thiol. The thioacetate $(4 \mathrm{mmol})$ was dissolved in anhydrous diethyl ether $(15 \mathrm{~mL})$ and cooled to $0{ }^{\circ} \mathrm{C}$. A solution of lithium aluminium hydride $(0.31 \mathrm{~g} ; 8 \mathrm{mmol})$ in anhydrous diethyl ether $(15 \mathrm{~mL})$ was slowly added and then stirred at RT for $2 \mathrm{~h}$. Anhydrous diethyl ether $(30 \mathrm{~mL})$, saturated ammonium chloride solution $(20 \mathrm{~mL})$, and $\mathrm{HCl}(2 \mathrm{~mol} / \mathrm{L} ; 20 \mathrm{~mL})$ were added at $0{ }^{\circ} \mathrm{C}$. The aqueous layer was washed twice with diethyl ether $(30 \mathrm{~mL})$ and the combined organic layers were washed twice with saturated sodium hydrogen carbonate solution $(20 \mathrm{~mL})$ and dried over sodium sulfate. The solvent was evaporated in vacuo and the residue was purified by column chromatography on silca gel using pentane as the eluent. 


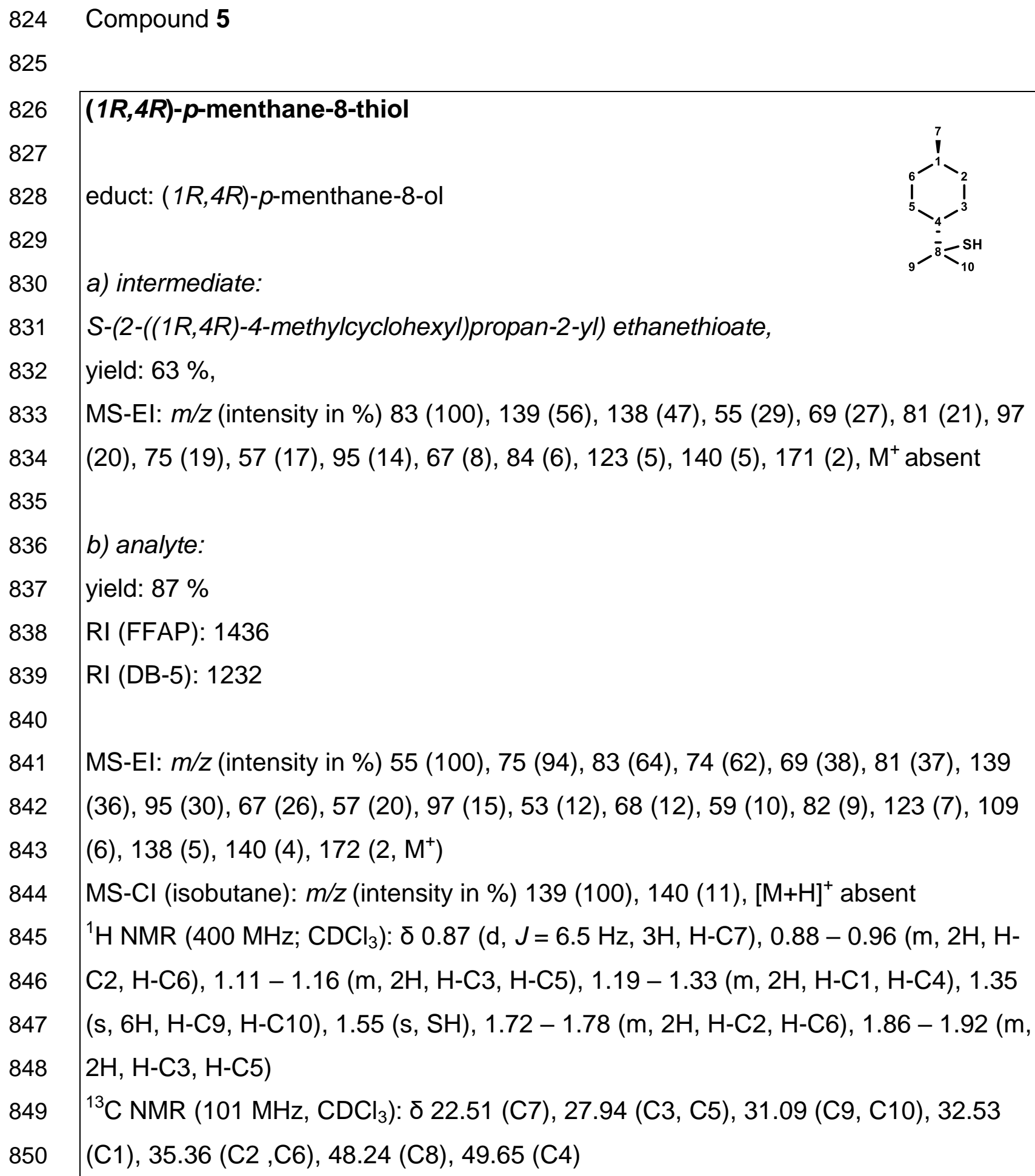

MS-El: m/z (intensity in \%) 55 (100), 75 (94), 83 (64), 74 (62), 69 (38), 81 (37), 139 842 (36), 95 (30), 67 (26), 57 (20), 97 (15), 53 (12), 68 (12), 59 (10), 82 (9), 123 (7), 109 843 (6), $138(5), 140(4), 172\left(2, M^{+}\right)$

844

MS-Cl (isobutane): $\mathrm{m} / \mathrm{z}$ (intensity in \%) $139(100), 140(11),[\mathrm{M}+\mathrm{H}]^{+}$absent

845

846

${ }^{1} \mathrm{H}$ NMR $\left(400 \mathrm{MHz} ; \mathrm{CDCl}_{3}\right): \delta 0.87(\mathrm{~d}, J=6.5 \mathrm{~Hz}, 3 \mathrm{H}, \mathrm{H}-\mathrm{C} 7), 0.88-0.96(\mathrm{~m}, 2 \mathrm{H}, \mathrm{H}-$ C2, H-C6), 1.11 - 1.16 (m, 2H, H-C3, H-C5), 1.19 - 1.33 (m, 2H, H-C1, H-C4), 1.35 (s, 6H, H-C9, H-C10), 1.55 (s, SH), $1.72-1.78$ (m, 2H, H-C2, H-C6), $1.86-1.92$ (m, 848 $2 \mathrm{H}, \mathrm{H}-\mathrm{C} 3, \mathrm{H}-\mathrm{C} 5)$

${ }^{13} \mathrm{C}$ NMR (101 MHz, CDCl $)$ ): $\delta 22.51$ (C7), 27.94 (C3, C5), 31.09 (C9, C10), 32.53

850 (C1), 35.36 (C2 ,C6), 48.24 (C8), 49.65 (C4) 


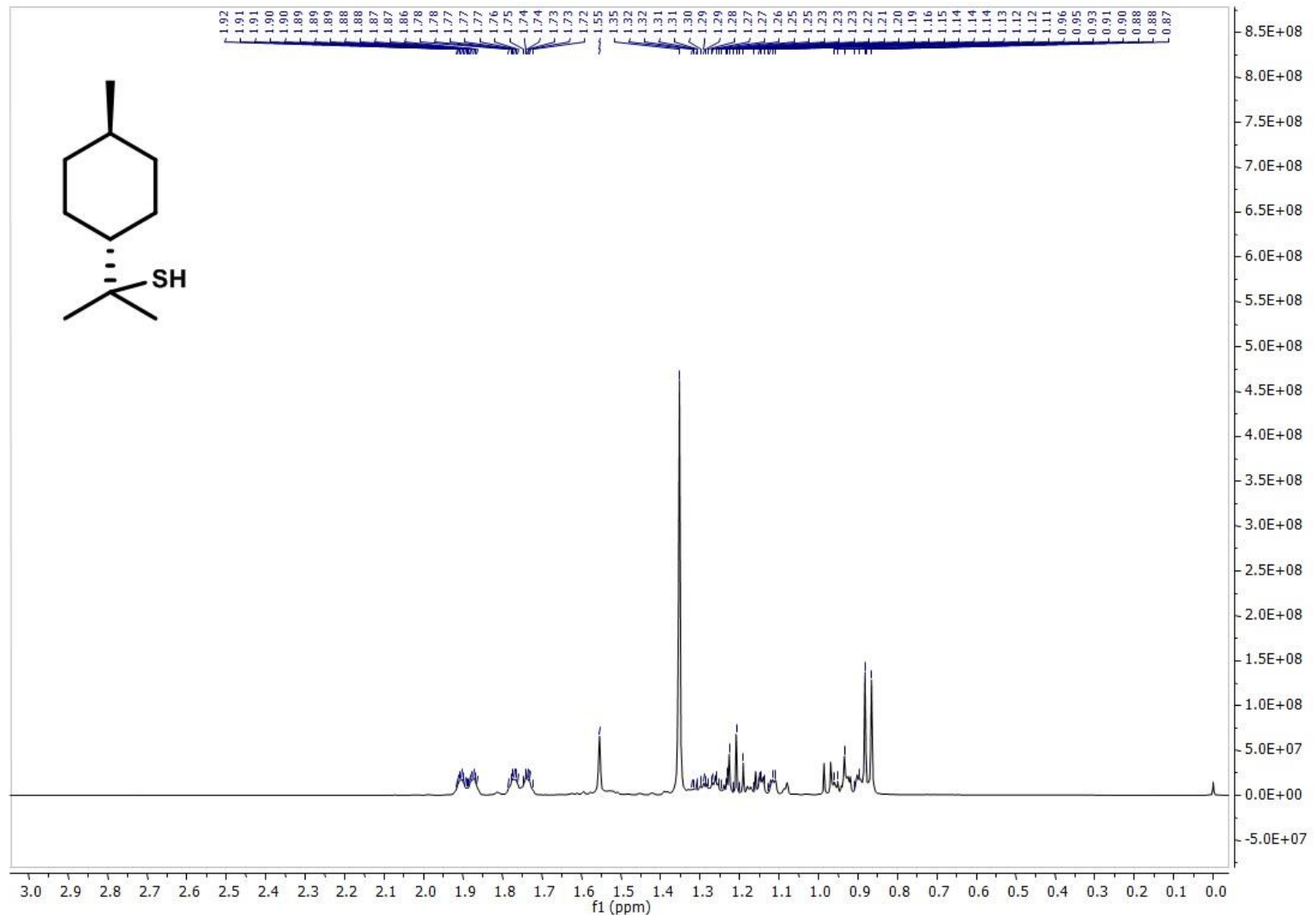

Figure $\mathrm{S} 38$. ${ }^{1} \mathrm{H}$ NMR spectrum of $(1 R, 4 R)$ - $p$-menthane-8-thiol.

853

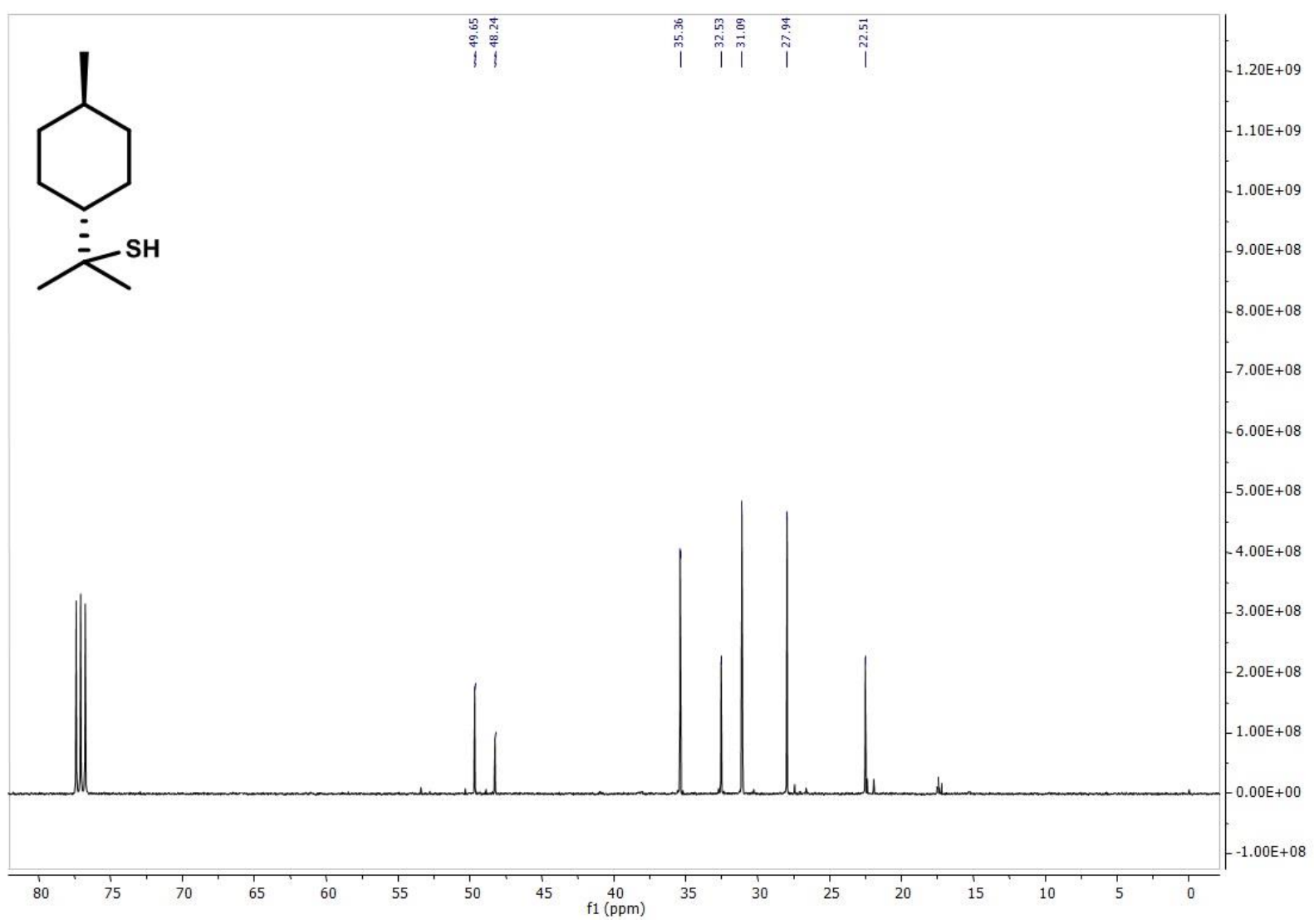

855 Figure S39. ${ }^{13} \mathrm{C}$ NMR spectrum of $(1 R, 4 R)$-p-menthane-8-thiol. 
856 Compound 10

857

858

p-menthane-4-thiol

859

860

educt: terpinen-4-ol

861

862

a) $1^{\text {st }}$ intermediate (obtained by hydrogenation):

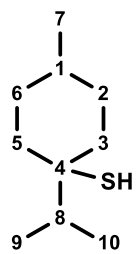

863

dihydroterpinen-4-ol, yield: $69 \%$,

864

MS-El: $m / z$ (intensity in \%) 113 (100), 95 (75), 43 (41), 99 (20), 41 (12), 67 (11), 55

865

(9), 114 (8), 111 (7), 57 (6), 69 (6), 96 (6), 70 (5), 81 (5), $156\left(1, \mathrm{M}^{+}\right)$

866

867

b) $2^{\text {nd }}$ intermediate:

868

S-(4-methyl-1-(propan-2-yl)cyclohexan-1-yl) ethanethioate,

869

yield: $27 \%$,

870

MS-El: $m / z$ (intensity in \%) 83 (100), 139 (45), 138 (40), 55 (38), 69 (31), 57 (22), 43

871

(20), 54 (19), 81 (19), 97 (19), 41 (16), 95 (12), 67 (8), 84 (6), 171 (2), M+ absent

872

873

b) analyte:

874

yield: $73 \%$

875

RI (FFAP): 1438

876

RI (DB-5): 1237

877

878

MS-El: $m / z$ (intensity in \%) 83 (100), 75 (94), 139 (70), 74 (68), 55 (64), 81 (37), 69

879

(36), 41 (30), 95 (23), 97 (23), 57 (22), 67 (18), 172 (12, M+), 43 (10), 59 (9), 77 (8),

880

82 (8), 53 (7), 76 (7), 123 (7), 79 (6), 84 (6), 96 (6), 173 (3)

881

MS-Cl (isobutane): $\mathrm{m} / \mathrm{z}$ (intensity in \%) $139(100), 140(11), 173\left(2,[\mathrm{M}+\mathrm{H}]^{+}\right)$

882

${ }^{1} \mathrm{H}$ NMR $\left(400 \mathrm{MHz} ; \mathrm{CDCl}_{3}\right): \delta 0.87(\mathrm{~d}, J=6.6 \mathrm{~Hz}, 3 \mathrm{H}, \mathrm{H}-\mathrm{C} 7), 0.88-0.97(\mathrm{~m}, 2 \mathrm{H}, \mathrm{H}-$

883

C3, H-C2), 1.08 - 1.18 (m, 2H, H-C2, H-C3), 1.22 - 1.33 (m, 2H, H-C1, H-C8), 1.35

884

(d, J = 0.6 Hz, 6H, H-C9, H-C10), 1.56 (s, SH), $1.72-1.79$ (m, 2H, H-C2, H-C6),

885

$1.86-1.92(\mathrm{~m}, 2 \mathrm{H}, \mathrm{H}-\mathrm{C} 3, \mathrm{H}-\mathrm{C} 5)$

886

${ }^{13} \mathrm{C} \mathrm{NMR}\left(101 \mathrm{MHz}, \mathrm{CDCl}_{3}\right): \delta 22.51$ (C7), 27.95 (C3, C5), 31.09 (C9, C10), 32.54

887

(C1), 35.37 (C2, C6), 48.26 (C4), 49.65 (C8) 


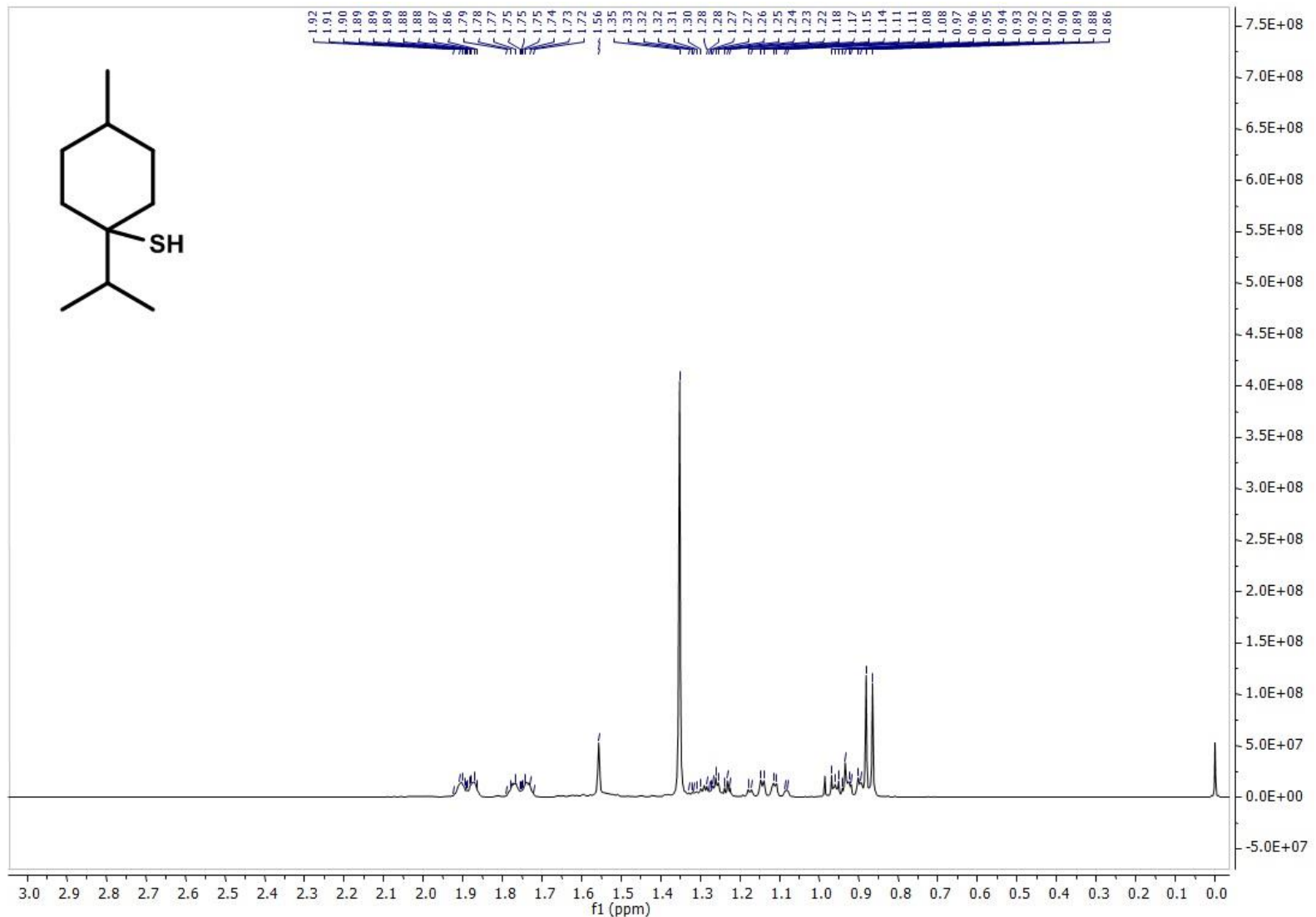

888

Figure S40. ${ }^{1} \mathrm{H}$ NMR spectrum of $p$-menthane-4-thiol.

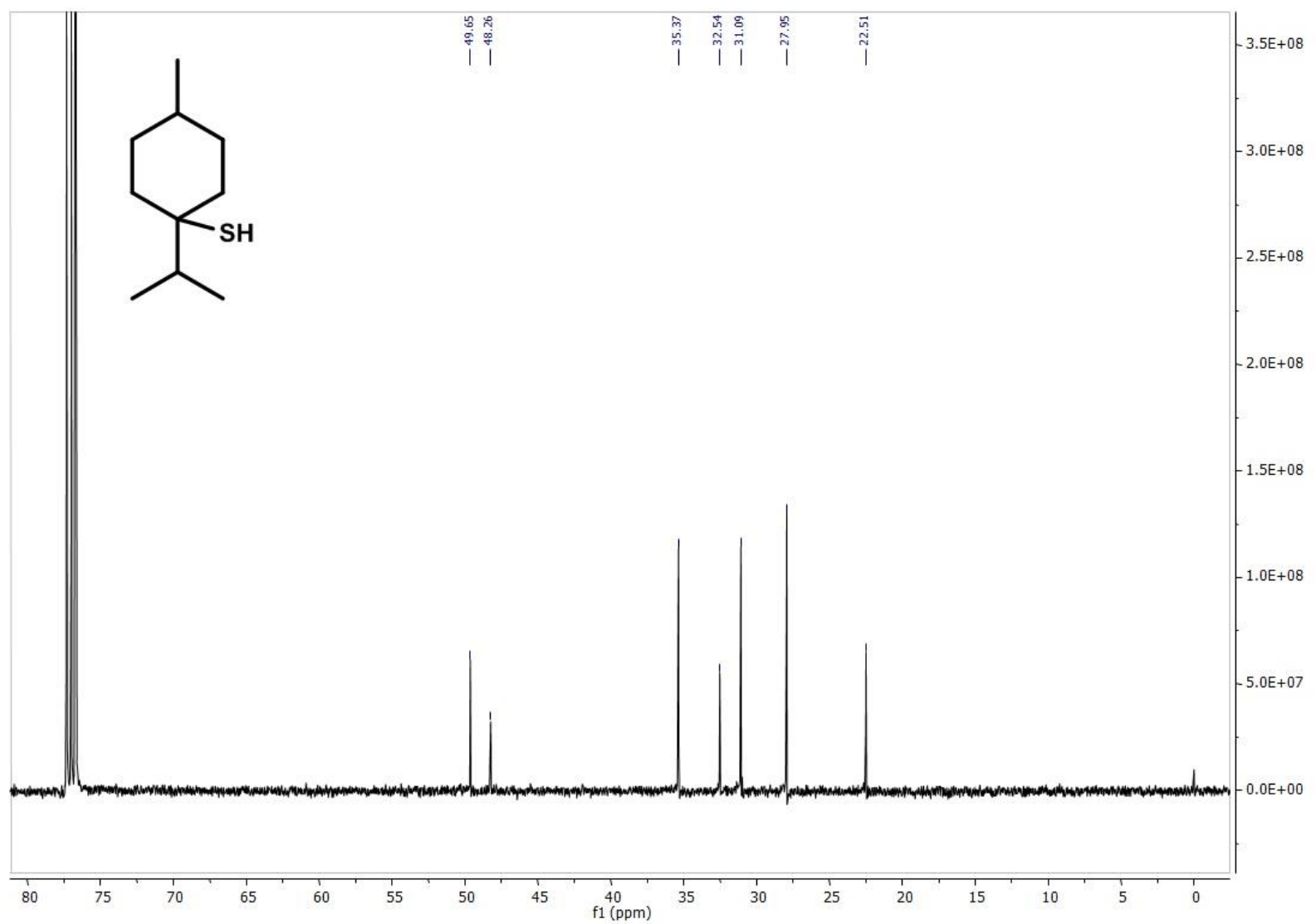

892 Figure S41. ${ }^{13} \mathrm{C}$ NMR spectrum of $p$-menthane-4-thiol. 
893

Compound 11

894

895

896

897

898

899

a) intermediate:

900

901

902

903

904

905

906

907

908

909

910

911

912

913

914

915

916

yield: $72 \%$,

b) analyte:

yield: $79 \%$

RI (DB-5): 1280

\section{p-cymene-8-thiol}

educt: $p$-cymene-8-ol

S-(2-(p-tolyl)propan-2-yl) ethanethioate

MS-El: $m / z$ (intensity in \%) 133 (100), 105 (40), 134 (13), 91 (12), 93 (11), 117 (8),

115 (6), 77 (5), $208\left(3, \mathrm{M}^{+}\right)$

RI (FFAP): 1722

MS-El: $m / z$ (intensity in \%) 133 (100), 105 (37), 117 (28), 91 (22), 132 (22), 115 (18), 93 (15), 134 (12), 41 (11), 65 (10), 77 (8), 59 (6), 131 (5), $166\left(2, \mathrm{M}^{+}\right)$

MS-Cl (isobutane): $m / z$ (intensity in \%) $133(100), 134(13),[\mathrm{M}+\mathrm{H}]^{+}$absent

${ }^{1} \mathrm{H}$ NMR (400 MHz; $\mathrm{CDCl}_{3}$ ): $\delta 1.81$ (s, 6H, H-C9, H-C10), 2.21 (s, SH), 2.32 (s, 3H, H-C7), 7.12 (d, J=8.0 Hz, 2H, H-C2, H-C6), 7.45 (d, J=8.3 Hz, 2H, H-C3, H-C5)

${ }^{13} \mathrm{C}$ NMR (101 MHz, CDCl 3 ): $\delta 20.96$ (C7), 34.56 (C9, C10), 45.70 (C8), 125.33 (C3, C5), 128.93 (C2, C6), 136.26 (C1), 145.59 (C4) 


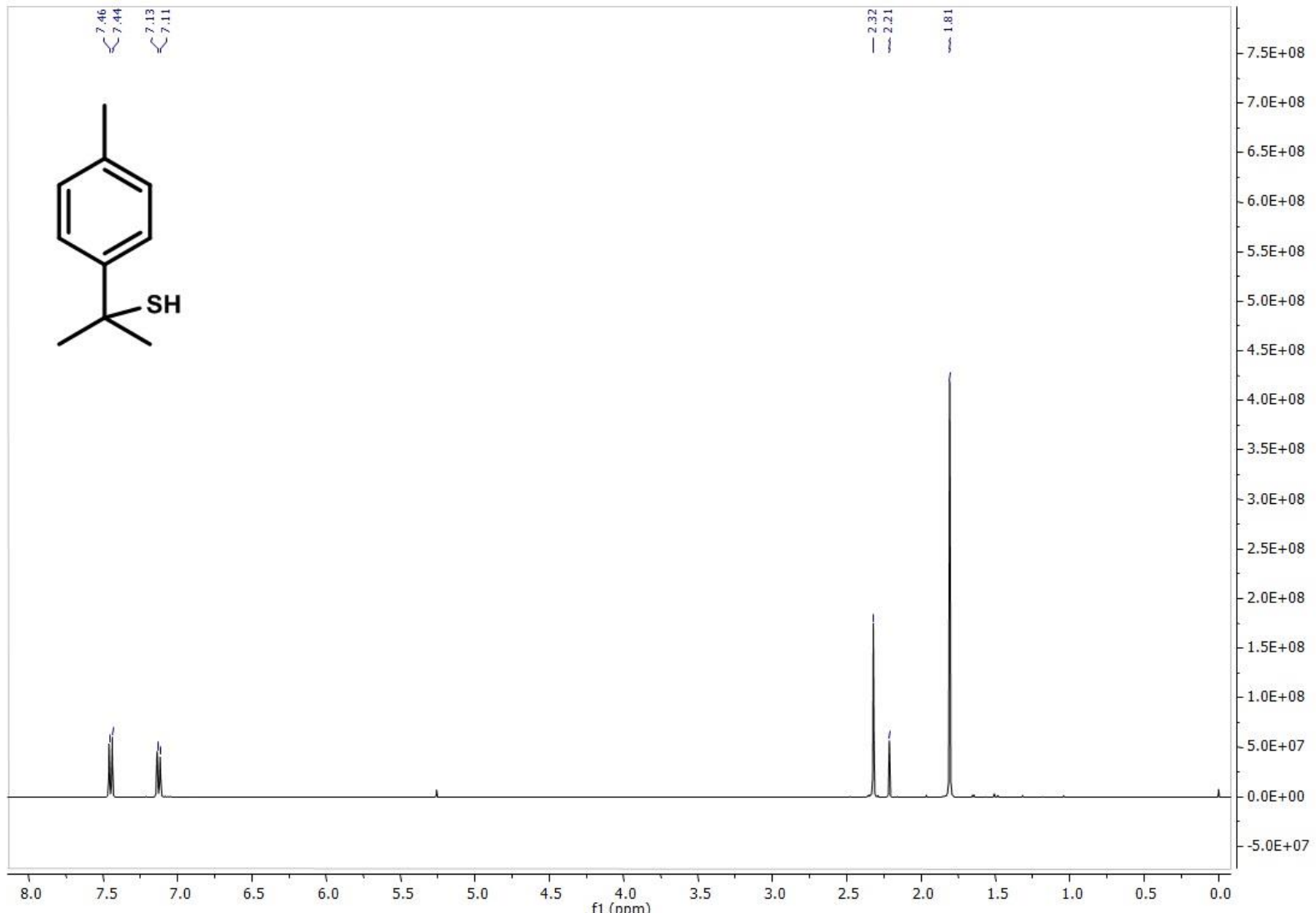

918 Figure S42. ${ }^{1} \mathrm{H}$ NMR spectrum of $p$-cymene-8-thiol.

919

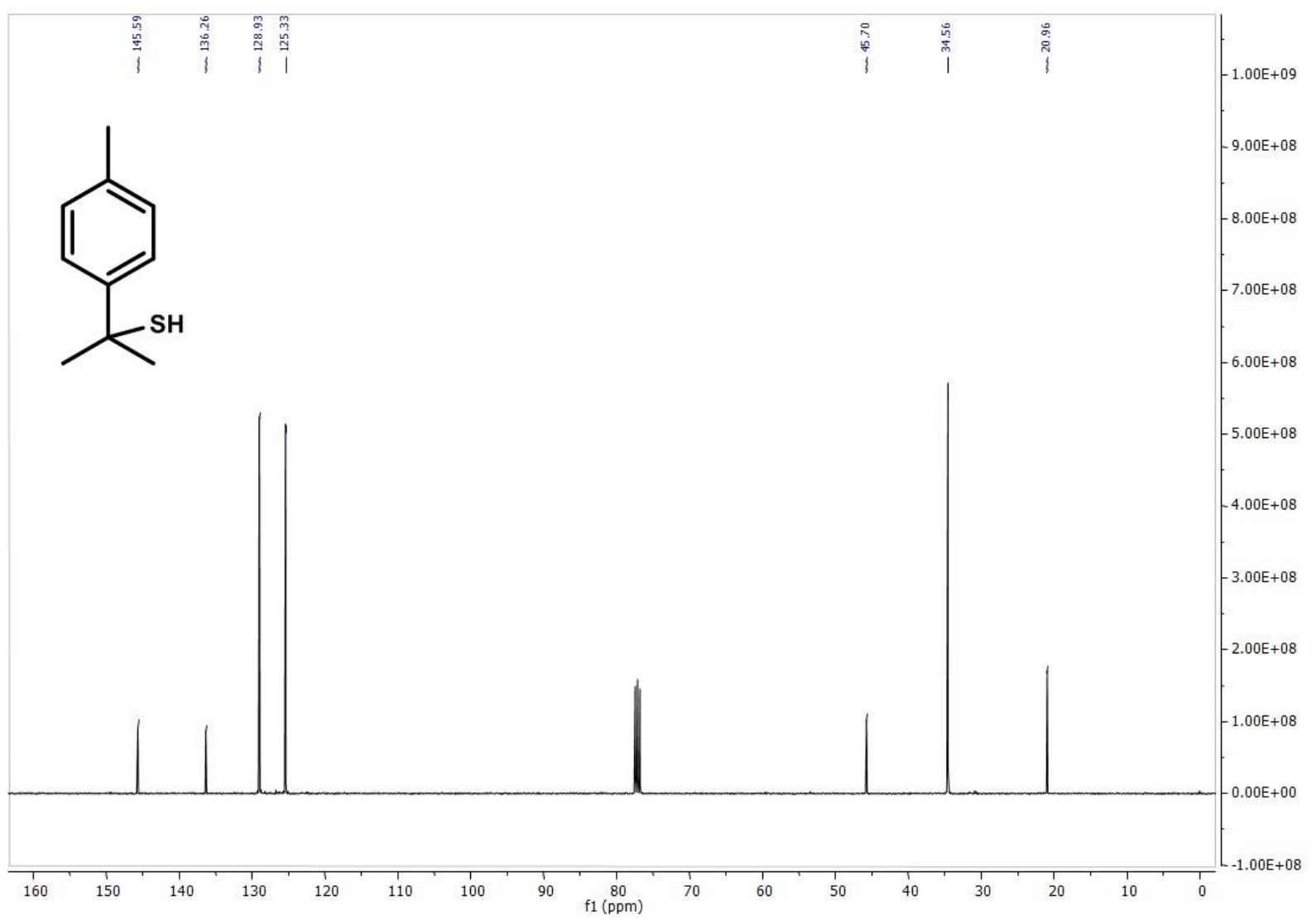

921 Figure S43. ${ }^{13} \mathrm{C}$ NMR spectrum of $p$-cymene-8-thiol. 
922 Compound 13

923

924

925

926 educt: $p$-cymene-7-ol

927

928

a) intermediate:

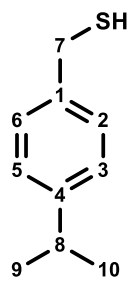

929

S-(4-(propan-2-yl)benzyl) ethanethioate,

930

yield: $29 \%$,

931

MS-El: $m / z$ (intensity in \%) 133 (100), 105 (28), 165 (17), 117 (16), 91 (14), 208 (14,

932

$\left.\mathrm{M}^{+}\right), 134(12), 164(8), 118(7), 115(6), 123(5)$

933

934

b) analyte:

935

yield: $19 \%$

936

RI (FFAP): 1857

937

RI (DB-5): 1346

938

939

MS-El: $m / z$ (intensity in \%) 133 (100), 105 (44), 117 (25), $166\left(24, \mathrm{M}^{+}\right), 91$ (21), 118

940

(16), 134 (14), 115 (13), 77 (13), 92 (10), 65 (8), 79 (7), 151 (5)

941

MS-Cl (isobutane): $m / z$ (intensity in \%) $133(100), 134(12), 167\left(6,[\mathrm{M}+\mathrm{H}]^{+}\right.$)

942

${ }^{1} \mathrm{H}$ NMR (400 MHz; $\mathrm{CDCl}_{3}$ ): $\delta 1.23$ (d, J = 6.9 Hz, 6H, H-C9, H-C10), 2.28 - 2.34 (m,

943

$\mathrm{SH}), 2.84-2.94$ (m, 1H, H-C8), 4.57 (s, 2H, H-C7), 7.19 (d, J=8.2 Hz, 2H, H-C3, H-

944

C5), 7.25 (d, J = 8.2 Hz, 2H, H-C2, H-C6)

945

${ }^{13} \mathrm{C}$ NMR (101 MHz, CDCl 3 ): $\delta 24.07$ (C9, C10), 33.91 (C8), 65.10 (C7), 126.62 (C3,

946

C5), 127.23 (C2, C6), 138.40 (C1), 148.40 (C4) 


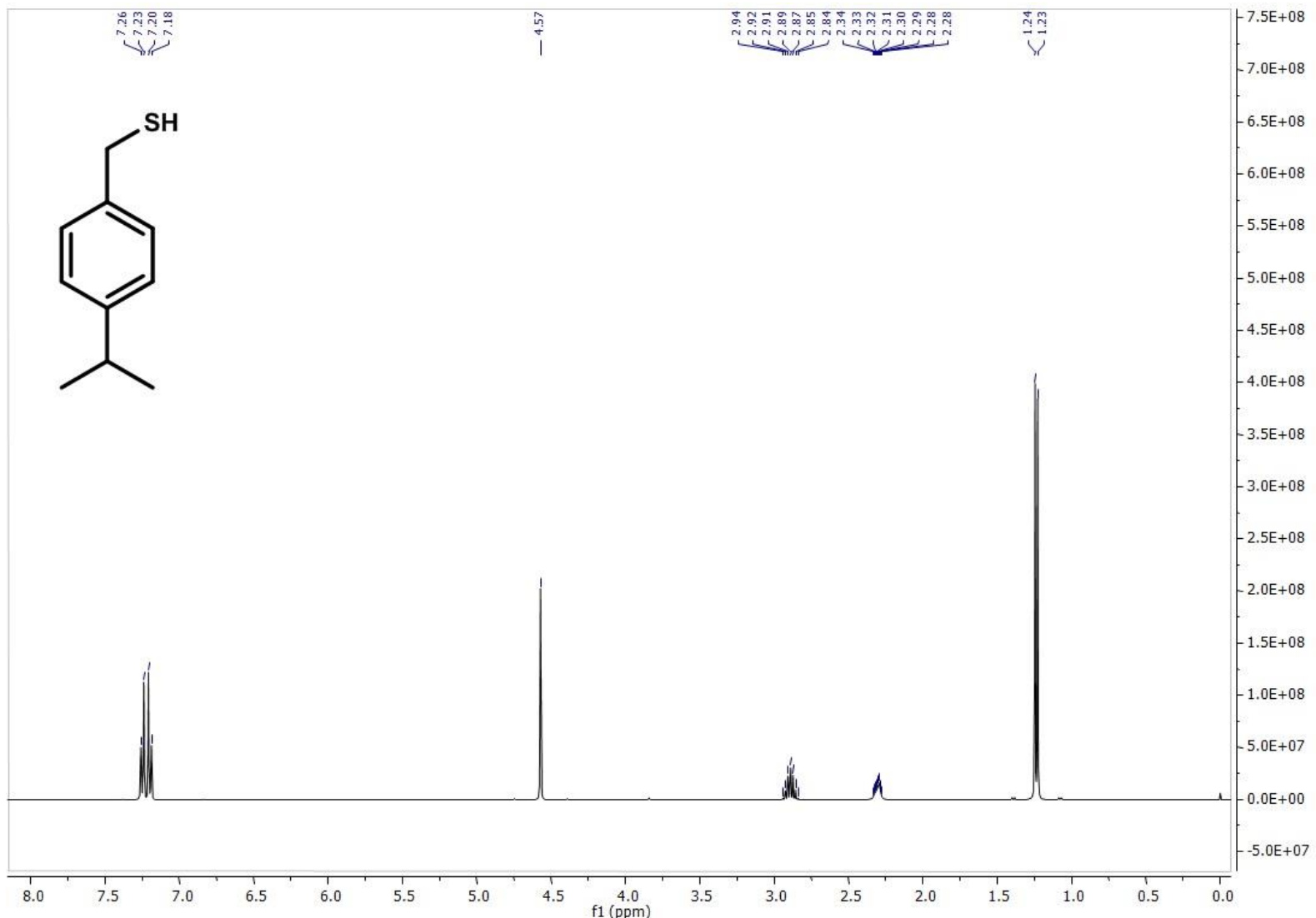

948 Figure S44. ${ }^{1} \mathrm{H}$ NMR spectrum of $p$-cymene-7-thiol.

949

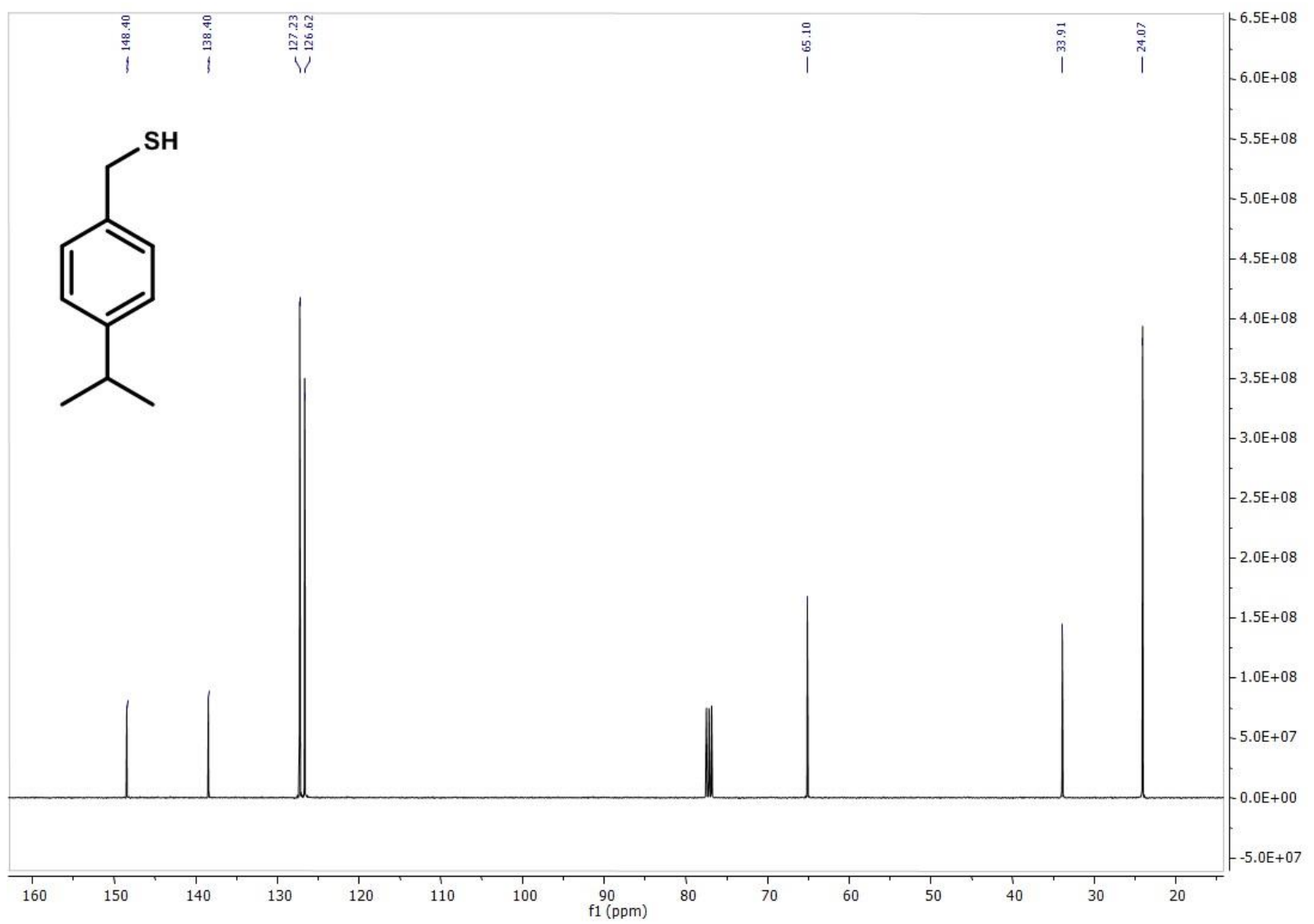

951 Figure S45. ${ }^{13} \mathrm{C}$ NMR spectrum of $p$-cymene-7-thiol. 
952 Compound 17

953

954

955

956

educt: (-)-carveol

957

958

a) intermediate:

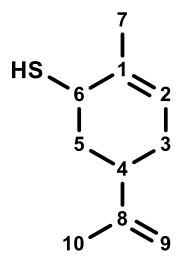

959

S-(2-methyl-5-(1-methylethenyl)cyclohex-2-en-1-yl) ethanethioate,

960

yield: $35 \%$,

961

MS-El: m/z (intensity in \%) 93 (100), 91 (58), 119 (44), 107 (40), 77 (38), 55 (37), 79

962

(32), 134 (31), 167 (29), 105 (25), 92 (22), 125 (21), 53 (19), 135 (18), 168 (16), 65

963

(16), 69 (15), 99 (14), 67 (10), 78 (9), $\mathrm{M}^{+}$absent

964

965

b) analyte:

966

yield: $83 \%$

967

RI (FFAP): 1650

968

RI (DB-5): 1279

969

970

MS-El: m/z (intensity in \%) 91 (100), 93 (78), 119 (78), 134 (66), 77 (53), 125 (45), 79

971

972

(41), 105 (41), 107 (39), 92 (36), 65 (28), 85 (25), 124 (24), 78 (17), 106 (16), 55 (15), 53 (14), 99 (13), 67 (12), 97 (10), 51 (9), 135 (7), 168 (6, M+)

973

MS-CI (isobutane): m/z (intensity in \%) 135 (100), 93 (23), 107 (22), 136 (12), 134

974

(7), $[\mathrm{M}+\mathrm{H}]^{+}$absent

975

976

977

978

979

${ }^{1} \mathrm{H}$ NMR (400 MHz; $\mathrm{CDCl}_{3}$ ): $\delta 1.74$ (d, J = 7.0 Hz, SH), 1.76 (s, 3H, H-C10), 1.81 (s, $3 \mathrm{H}, \mathrm{H}-\mathrm{C} 7), 1.91$ - 1.92 (m, 1H, H-C3), 1.93 - 1.95 (m, 2H, H-C5), $2.12-2.20(\mathrm{~m}, 1 \mathrm{H}$, H-C3), $2.50-2.57$ (m, 1H, H-C4), $3.43-3.45$ (m, 1H, H-C6), 4.73 (d, J=1.0 Hz, 1H, $\mathrm{H}-\mathrm{C} 9), 4.76$ (d, J=1.0 Hz, 1H, H-C9), $5.42-5.44(\mathrm{~m}, 1 \mathrm{H}, \mathrm{H}-\mathrm{C} 2)$

${ }^{13} \mathrm{C}$ NMR (101 MHz, CDCl $)$ ): $\delta 20.97$ (C10), 21.90 (C7), 30.73 (C3), 34.93 (C4),

980

37.83 (C5), 40.11 (C6), 109.00 (C9), 123.44 (C2), 135.21 (C1), 149.10 (C8) 


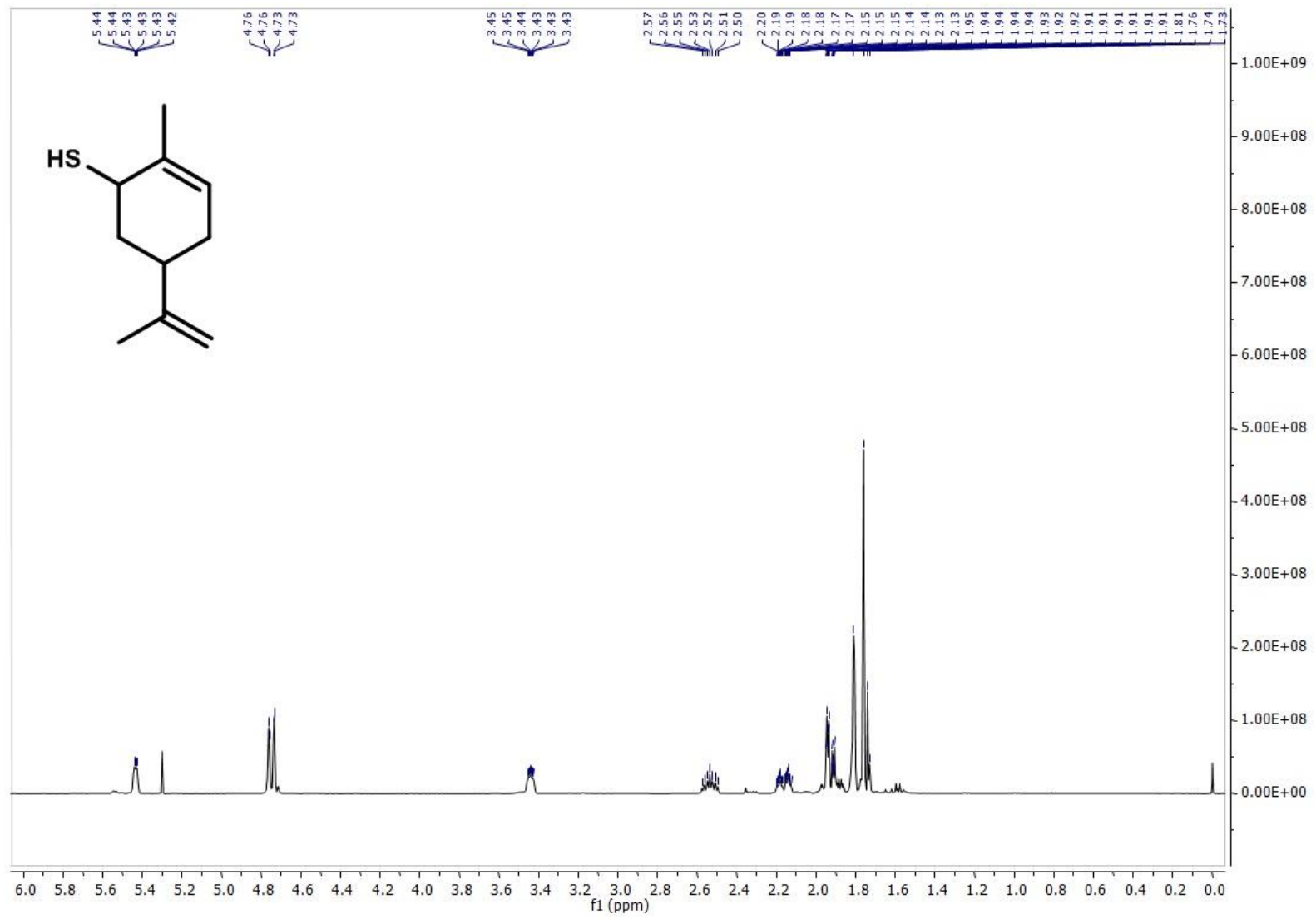

Figure S46. ${ }^{1} \mathrm{H}$ NMR spectrum of $(4 R)-1,8-p$-menthadiene-6-thiol.

983

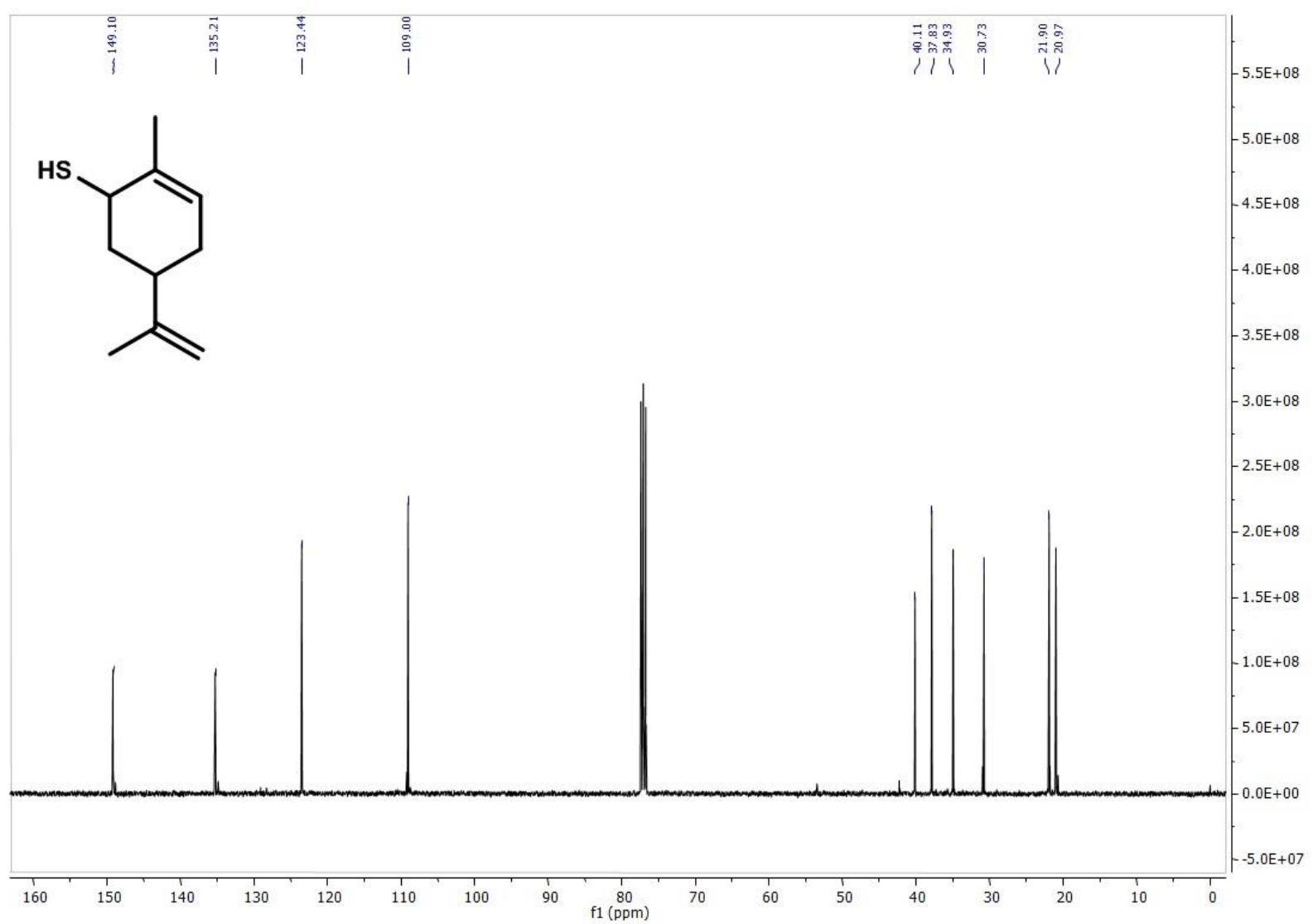

985 Figure S47. ${ }^{13} \mathrm{C}$ NMR spectrum of (4R)-1,8- $p$-menthadiene-6-thiol. 
986 Compound 23

987

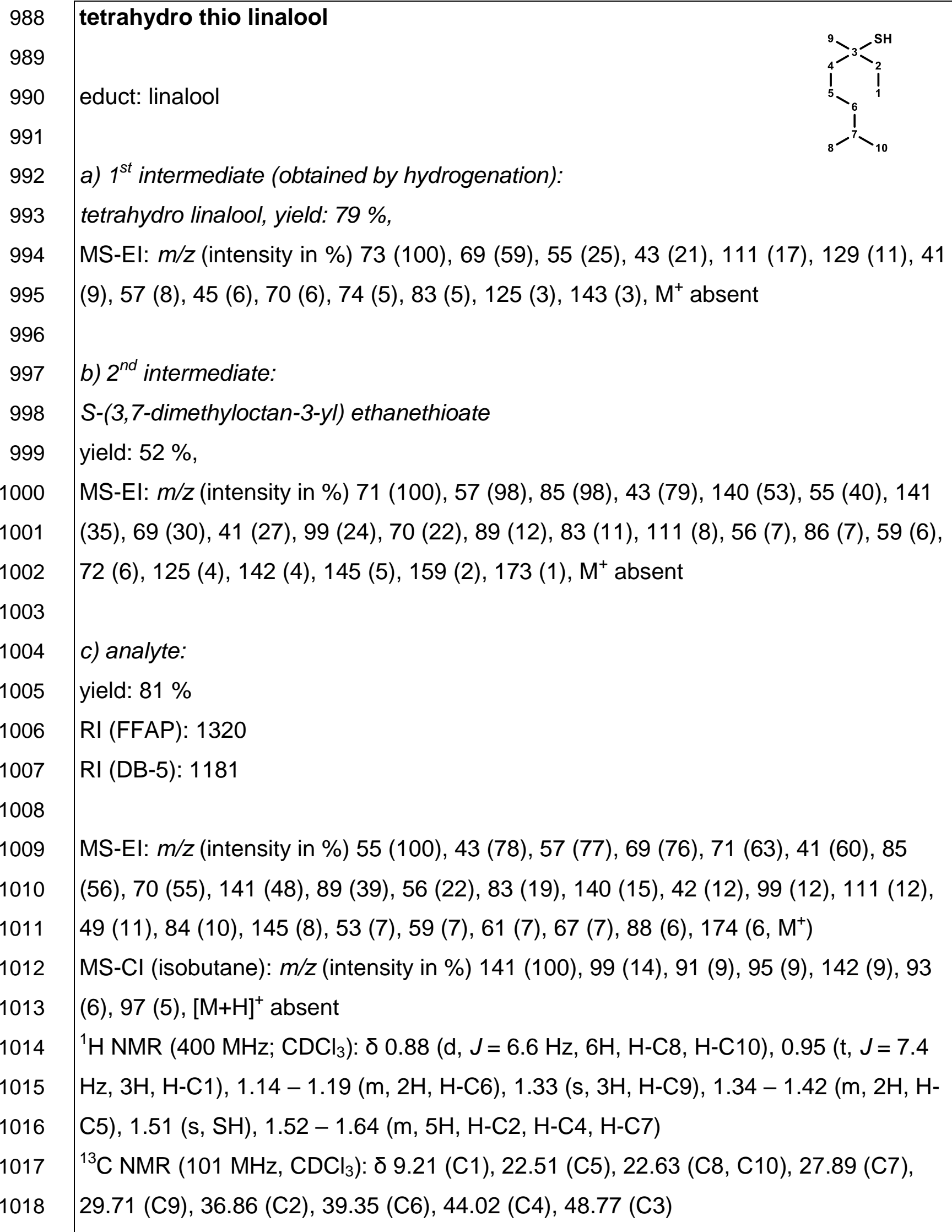




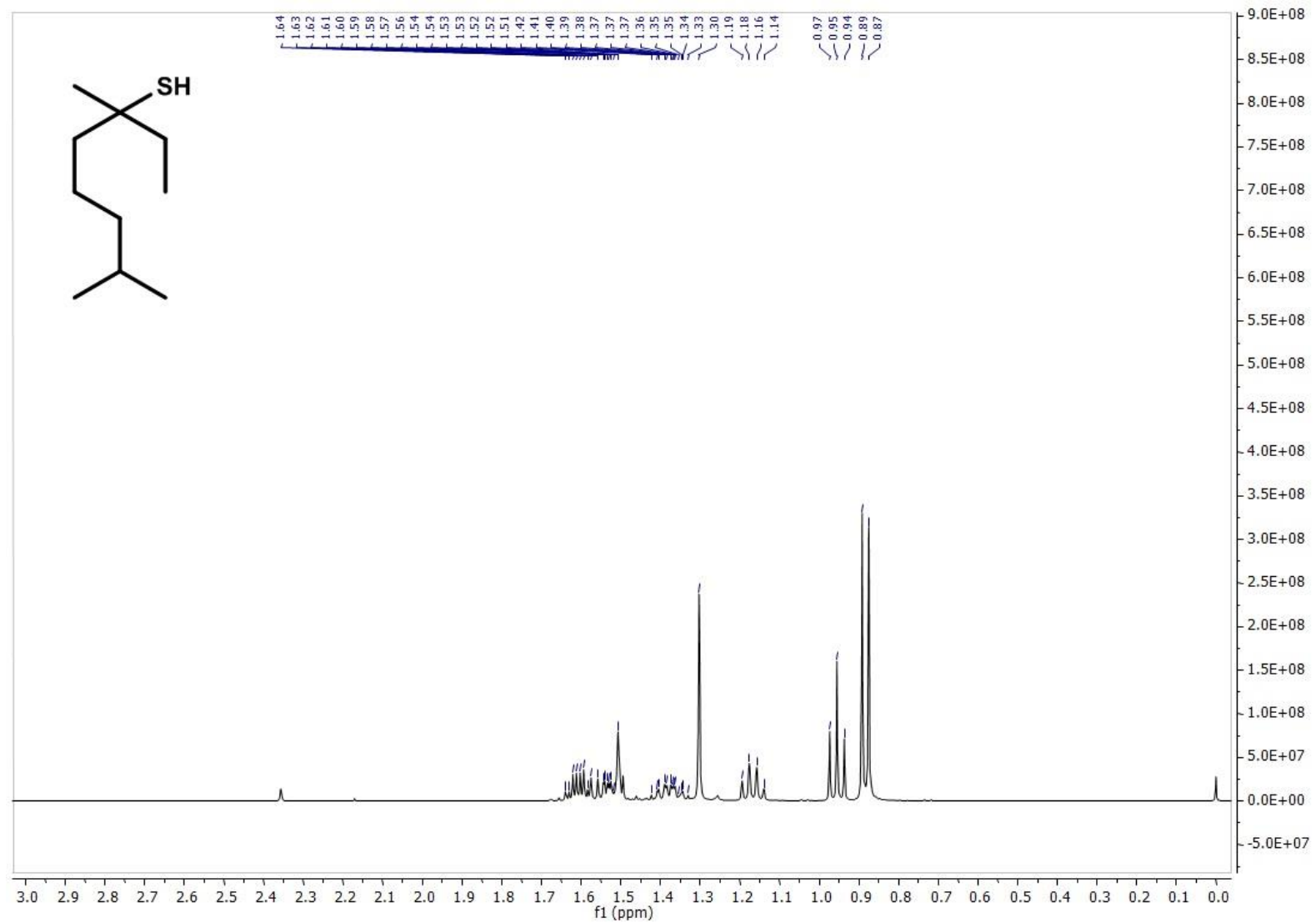

1020 Figure S48. ${ }^{1} \mathrm{H}$ NMR spectrum of tetrahydro thio linalool.

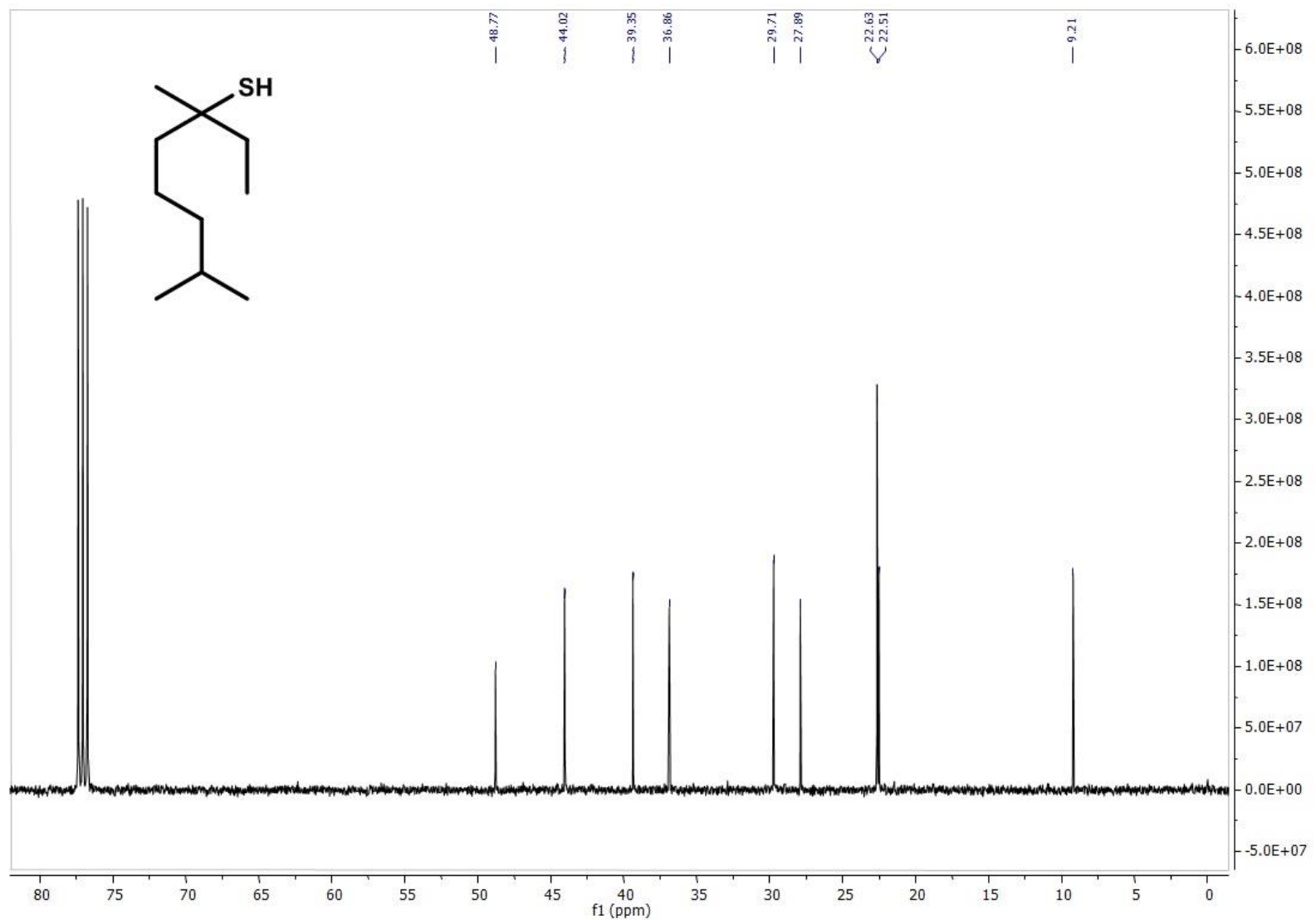

1023 Figure S49. ${ }^{13} \mathrm{C}$ NMR spectrum of tetrahydro thio linalool. 
1024 Compound 24

1025

1026

tetrahydro thio myrcenol

1027

1028

educt: tetrahydro myrcenol

1029

1030

a) intermediate:

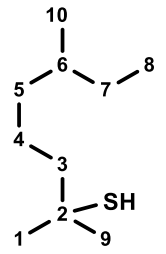

1031

S-(2,6-dimethyloctan-2-yl) ethanethioate

1032

yield: $20 \%$,

1033

MS-El: m/z (intensity in \%) 57 (100), 71 (71), 43 (68), 85 (68), 41 (42), 55 (36), 69

1034

(36), 140 (29), 141 (28), 56 (26), 70 (16), 75 (14), 99 (13), 83 (12), 84 (9), 111 (7),

1035

$142(6), \mathrm{M}^{+}$absent

1036

1037

b) product:

1038

yield: $93 \%$

1039

RI (FFAP): 1310

1040

RI (DB-5): 1177

1041

1042

MS-El: m/z (intensity in \%) 57 (100), 41 (83), 43 (80), 69 (77), 55 (76), 71 (66), 85

1043

(61), 56 (59), 75 (76), 79 (48), 141 (41), 83 (22), 140 (20), 84 (15), 99 (12), 111 (12),

1044

42 (10), 67 (10), 74 (10), 53 (9), 59 (8), $142(5), 174\left(5, \mathrm{M}^{+}\right)$

1045

MS-Cl (isobutane): $m / z$ (intensity in \%) 141 (100), 99 (14), $142(12),[\mathrm{M}+\mathrm{H}]^{+}$absent

1046

${ }^{1} \mathrm{H}$ NMR (400 MHz; $\left.\mathrm{CDCl}_{3}\right): \delta 0.85-0.88(\mathrm{~m}, 6 \mathrm{H}, \mathrm{H}-\mathrm{C} 8, \mathrm{H}-\mathrm{C} 10), 1.06-1.18(\mathrm{~m}, 1 \mathrm{H}$,

1047

$\mathrm{H}-\mathrm{C} 5, \mathrm{H}-\mathrm{C} 7$ ), 1.27 - 1.39 (m, 4H, H-C4, H-C5, H-C6, H-C7), 1.37 (s, 6H, H-C1, H-

1048

C9), $1.40-1.46$ (m, 1H, H-C4), $1.51-1.56$ (m, 2H, H-C3), 1.68 (s, SH)

1049

${ }^{13} \mathrm{C}$ NMR (101 MHz, $\mathrm{CDCl}_{3}$ ): $\delta 11.41$ (C8), 19.20 (C10), 22.84 (C4), 29.50 (C7),

1050

32.86 (C1, C9), 34.33 (C6), 36.91 (C5), 44.84 (C2), 46.86 (C3) 


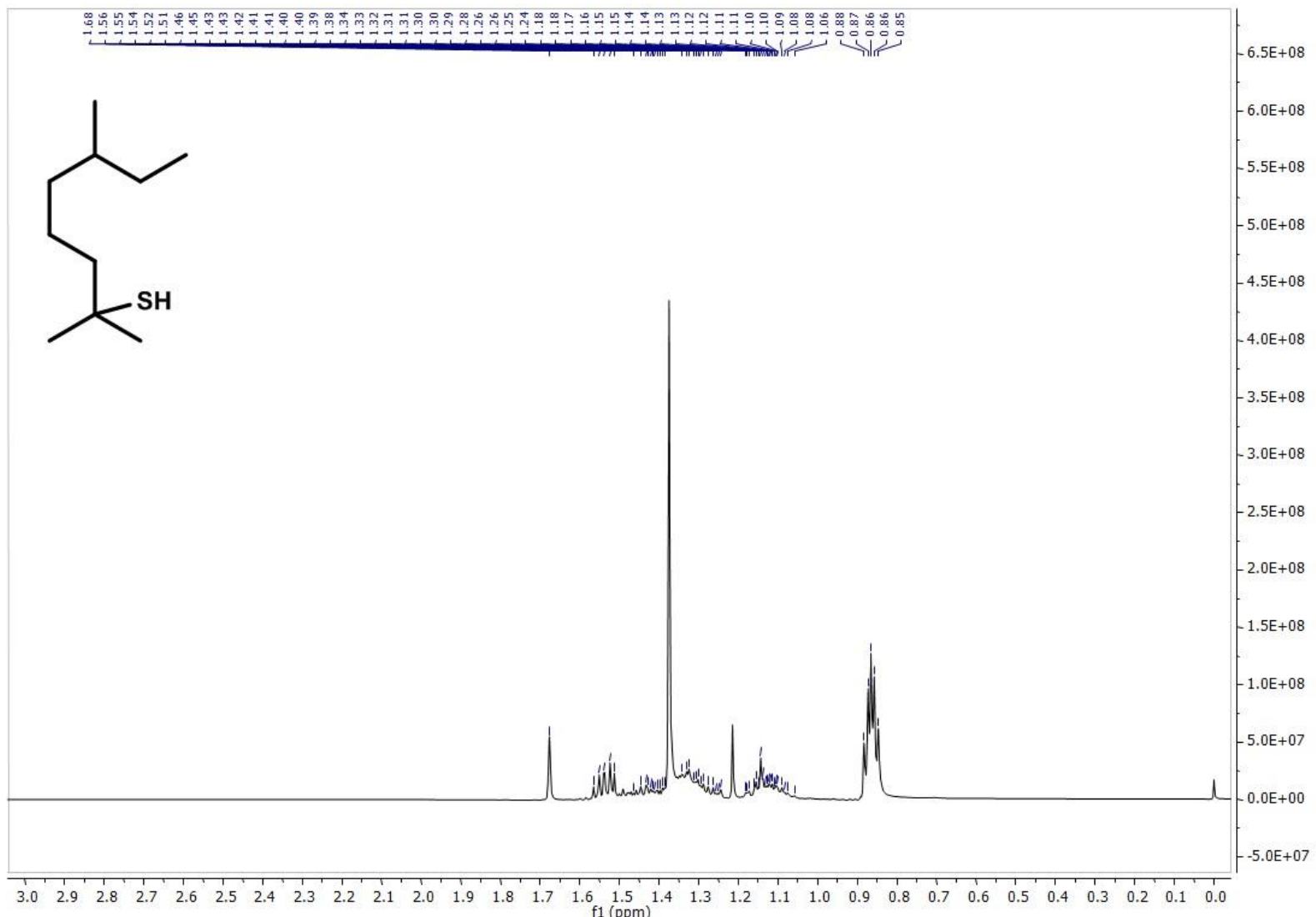

Figure S50. ${ }^{1} \mathrm{H}$ NMR spectrum of tetrahydro thio myrcenol.

1053

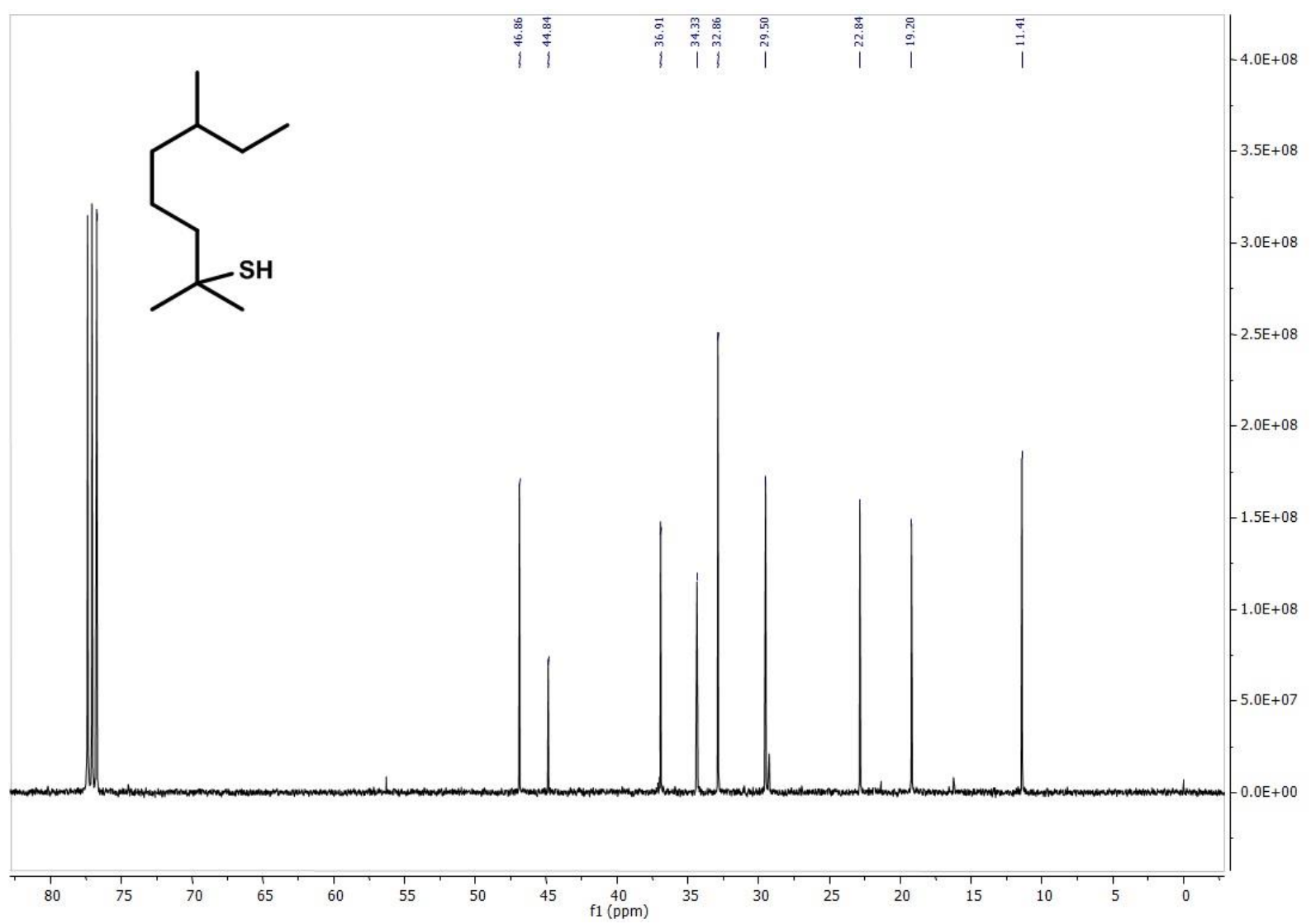

1055 Figure S51. ${ }^{13} \mathrm{C}$ NMR spectrum of tetrahydro thio myrcenol. 
1056 Synthetic route used in the preparation of two diastereomers of 1-p-menthene1057 3-thiol (compounds 4a and 4b) from linalool using synthetic route $B$ 1058

1059 The two diastereomers of 1-p-menthene-3-thiol were synthesized using synthetic 1060 route $B$. 
1061

Compound 4a

1062

1063

1064

1065

1066

1067

1068

1069

1070

1071

1072

1073

1074

1075

1076

1077

1078

1079

1080

1081

1082

1083

1084

1085

1086

1087

1088

1089

educt: linalool

yield: $22 \%$,

b) analyte:

yield: $22 \%$

\section{1-p-menthene-3-thiol, diastereomer 1}

a) intermediate:

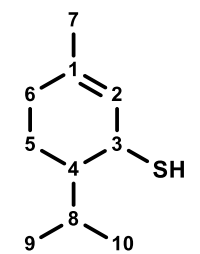

S-(3-methyl-6-(propan-2-yl)cyclohex-2-en-1-yl) ethanethioate,

diastereomer 2

MS-El: m/z (intensity in \%) 81 (100), 93 (74), 137 (54), 91 (32), 77 (30), 95 (22), 43 (20), 69 (20), 79 (20), 136 (16), 41 (15), 121 (11), 92 (10), 94 (9), 55 (6), 67 (6), 82 (5), $105(5), 138(5), 170(1), 212\left(1, M^{+}\right)$

RI (FFAP): 1533

RI (DB-5): 1268

MS-El: m/z (intensity in \%) 81 (100), 137 (82), 93 (79), 91 (38), 77 (36), 95 (32), 69 (30), 79 (24), 41 (22), 43 (15), 121 (14), 136 (13), 92 (12), 67 (11), 94 (10), 55 (9), 82 (8), 53 (7), 65 (7), 85 (7), 138 (7), 119 (6), $170\left(2, \mathrm{M}^{+}\right)$

MS-Cl (isobutane): $\mathrm{m} / \mathrm{z}$ (intensity in \%) $137(100), 138(11), 171\left(2,[\mathrm{M}+\mathrm{H}]^{+}\right)^{1} \mathrm{H}$ NMR (400 MHz; $\mathrm{CDCl}_{3}$ ): 80.91 (d, J = 6.6 Hz, 3H, H-C9), 0.97 (d, J = $6.7 \mathrm{~Hz}, 3 \mathrm{H}, \mathrm{H}-\mathrm{C} 10$ ), $1.18-1.24$ (m, 1H, H-C4), 1.22 (d, J= 7.2 Hz, SH), $1.30-1.35$ (m, 1H, H-C5), 1.59 $-1.66(\mathrm{~m}, 1 \mathrm{H}, \mathrm{H}-\mathrm{C} 8), 1.65$ (s, 3H, H-C7), $1.73-1.77$ (m, 1H, H-C5), $1.95-2.00$ (m, $1 \mathrm{H}, \mathrm{H}-\mathrm{C} 6), 3.58-3.62(\mathrm{~m}, 1 \mathrm{H}, \mathrm{H}-\mathrm{C} 3), 5.58-5.61(\mathrm{~m}, 1 \mathrm{H}, \mathrm{H}-\mathrm{C} 2)$

${ }^{13} \mathrm{C} \mathrm{NMR} \mathrm{(101} \mathrm{MHz,} \mathrm{CDCl} 3$ ): $\delta 20.63$ (C9), 20.69 (C10), 20.71 (C5), 23.17 (C7), 29.58 (C4), 31.29 (C6), 39.29 (C3), 45.51 (C4), 125.16 (C2), 135.05 (C1) 
1090

1091

1092

1093

1094

1095

1096

1097

1098

1099

1100

1101

1102

1103

1104

1105

1106

1107

1108

1109

1110

1111

1112

1113

1114

1115

1116

1117

1118

Compound $\mathbf{4 b}$

\section{1-p-menthene-3-thiol, diastereomer 2}

educt: linalool

a) intermediate:

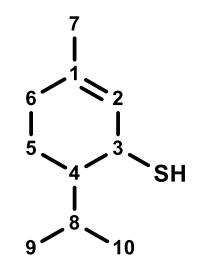

S-(3-methyl-6-(propan-2-yl)cyclohex-2-en-1-yl) ethanethioate,

diastereomer 1

yield: $43 \%$,

MS-El: m/z (intensity in \%) 81 (100), 93 (86), 137 (76), 91 (34), 77 (27), 95

(22), 136 (19), 69 (17), 79 (19), 43 (14), 121 (12), 41 (11), 92 (9), 138 (8),

119 (7), 55 (5), 67 (5), 94 (5), 105 (5), 170 (1), $212\left(1, \mathrm{M}^{+}\right)$

b) analyte:

yield: $59 \%$

RI (FFAP): 1544

RI (DB-5): 1280

MS-El: m/z (intensity in \%) 81 (100), 137 (88), 93 (63), 91 (27), 95 (27), 77 (25), 69 (22), 79 (18), 136 (12), 41 (12), 121 (10), 43 (9), 67 (8), 92 (8), 94 (7), 55 (6), 138 (6), $82(5), 119(5), 170\left(3, \mathrm{M}^{+}\right)$

MS-Cl (isobutane): $m / z$ (intensity in \%) $137(100), 138(12), 171\left(1,[\mathrm{M}+\mathrm{H}]^{+}\right.$)

${ }^{1} \mathrm{H}$ NMR (400 MHz; $\mathrm{CDCl}_{3}$ ): $\delta 0.82(\mathrm{~d}, J=6.9 \mathrm{~Hz}, 3 \mathrm{H}, \mathrm{H}-\mathrm{C} 9), 0.96$ (d, J=6.8 Hz, 3H, H-C10), $1.30-1.45$ (m, 2H, H-C4, H-C5), 1.55 (d, J = 7.8 Hz, SH), 1.66 (s, 3H, HC7), $1.78-1.84$ (m, 1H, H-C5), $1.91-1.96$ (m, 2H, H-C6), $2.01-2.06(m, 1 \mathrm{H}, \mathrm{H}-$ C8), $3.36-3.41$ (m, 1H, H-C3), $5.39-5.42$ (m, 1H, H-C2)

${ }^{13} \mathrm{C}$ NMR (101 MHz, $\mathrm{CDCl}_{3}$ ): $\delta 17.47$ (C9), 21.17 (C5), 21.58 (C10), 23.39 (C7), 27.46 (C8), 29.07 (C6), 39.67 (C3), 49.06 (C4), 123.38 (C2), 135.47 (C1) 


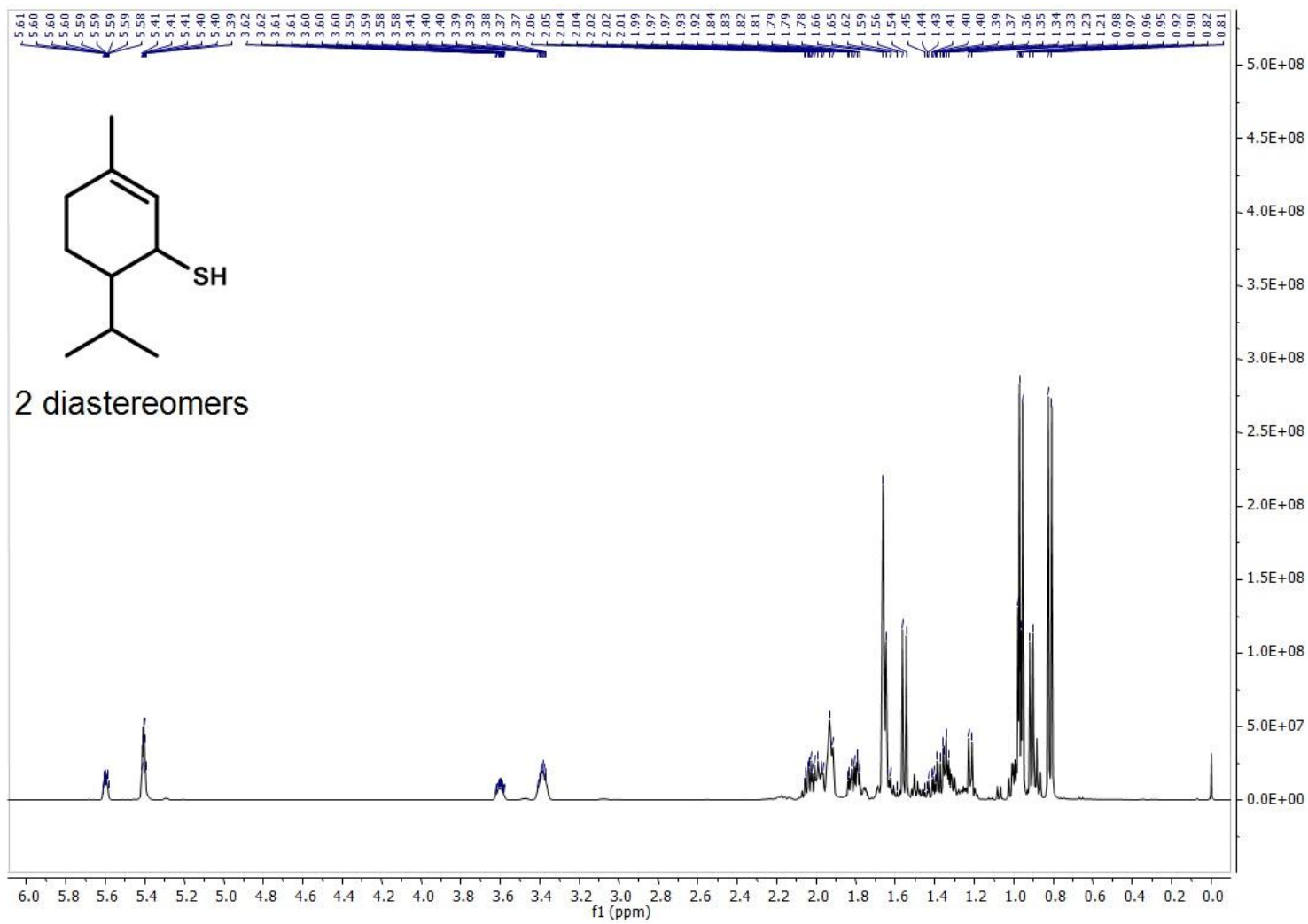

Figure S52. ${ }^{1} \mathrm{H}$ NMR spectrum of 1-p-menthene-3-thiol (mixture of 2 diastereomers).

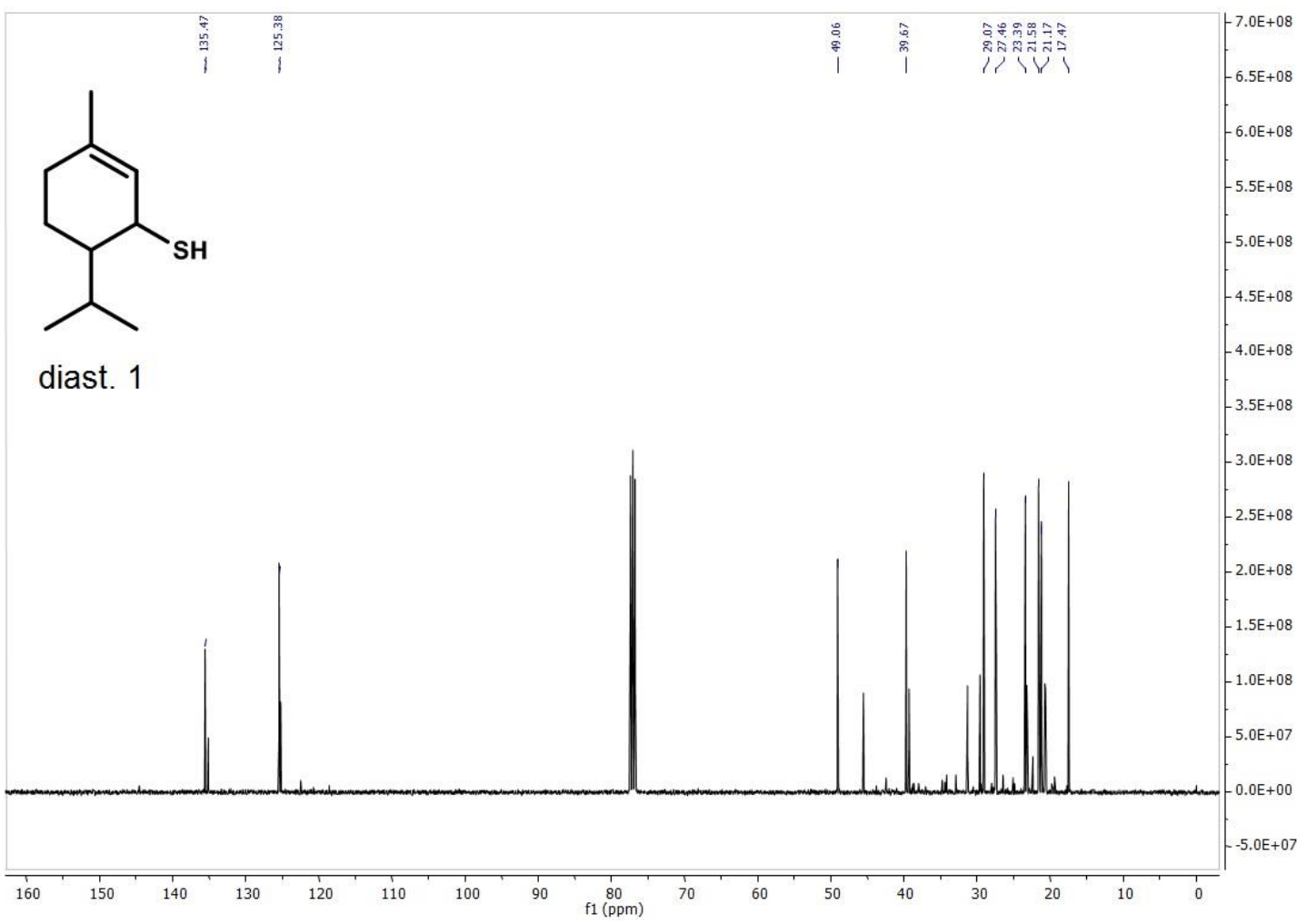

1123 Figure S53. ${ }^{13} \mathrm{C}$ NMR spectrum of 1-p-menthene-3-thiol (mixture of 2 1124 diastereomers); signals of diastereomer 1. 


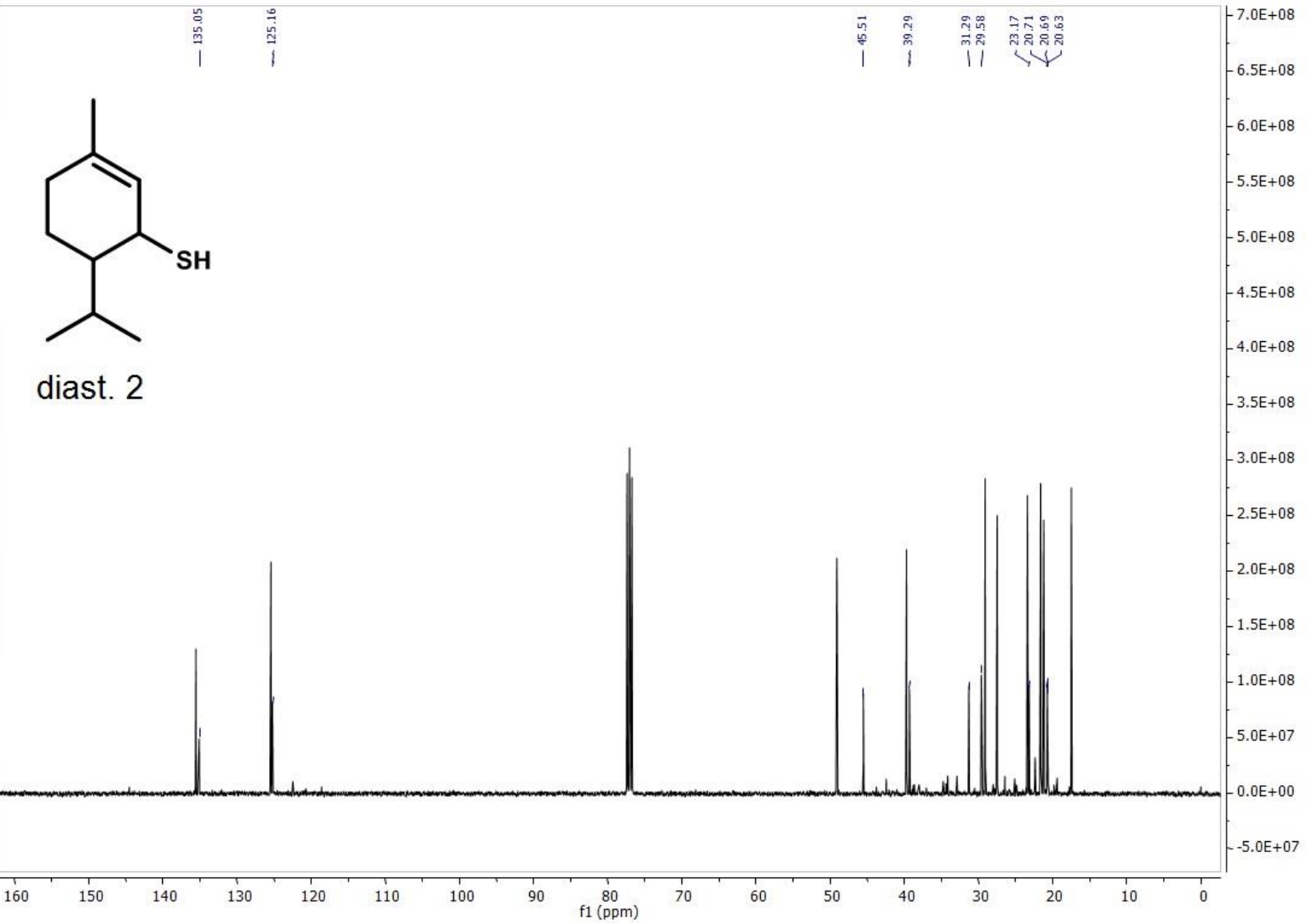

1125

Figure S54. ${ }^{13} \mathrm{C}$ NMR spectrum of 1-p-menthene-3-thiol (mixture of 2

1127 diastereomers); signals of diastereomer 2. 


\section{Synthesis of 1-p-menthene-4-thiol (3)}

1-p-Menthene-4-thiol was synthesized by epoxidation of terpinolene, reaction to the episulfide, and reduction, as shown in Figure S55.<smiles>CC1=CCC(=C(C)C)CC1</smiles>
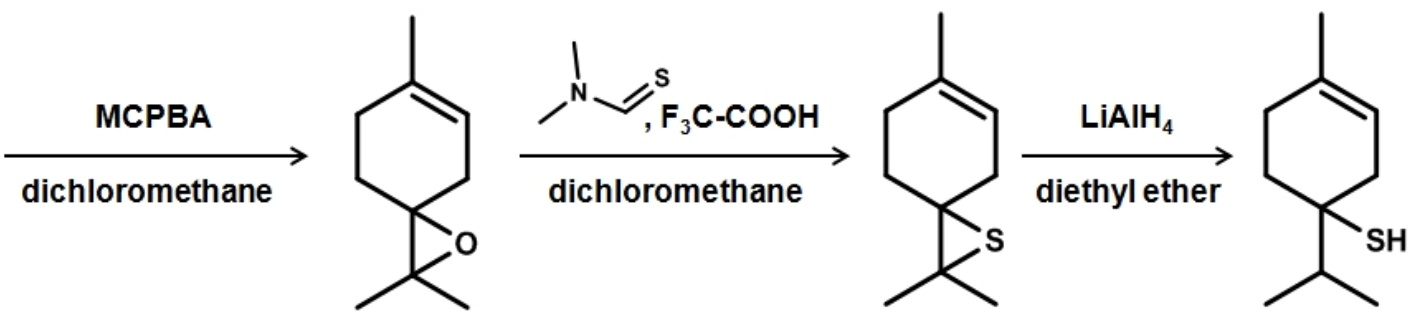

Figure S55. Synthetic strategy used for the preparation of 1-p-menthene-4-thiol.

a) Epoxidation of terpinolene. Terpinolene $(1.36 \mathrm{~g} ; 10 \mathrm{mmol})$ was dissolved in anhydrous dichloromethane $(20 \mathrm{~mL})$ and 3-chloroperoxybenzoic acid $(77 \%)(2.36 \mathrm{~g}$; $10 \mathrm{mmol})$ in anhydrous dichloromethane $(30 \mathrm{~mL})$ was dropwise added. The solution was stirred for $30 \mathrm{~min}$ at $0{ }^{\circ} \mathrm{C}$ and for further $30 \mathrm{~min}$ at RT. After the addition of an aqueous sodium carbonate solution $(10 \% ; 20 \mathrm{~mL})$, the reaction mixture was stirred for $30 \mathrm{~min}$. The organic layer was separated and washed twice with water $(50 \mathrm{~mL})$ before dried over sodium sulfate. The solvent was removed under reduced pressure and the crude product was purified by means of column chromatography on silica gel with pentane as the eluent.

b) Reaction to the episulfide. The epoxide $(1.18 \mathrm{~g} ; 7 \mathrm{mmol})$ was dissolved in anhydrous dichloromethane $(20 \mathrm{~mL})$ and $\mathrm{N}, \mathrm{N}$-dimethyl thioformamide $(1.25 \mathrm{~g}$; $14 \mathrm{mmol}$ ) was added. After addition of trifluoroacetic acid $(0.08 \mathrm{~g} ; 0.7 \mathrm{mmol})$ the reaction mixture was stirred for $3 \mathrm{~h}$ at $60^{\circ} \mathrm{C}$. The solvent was removed under reduced pressure, the residue was dissolved in pentane $(30 \mathrm{~mL})$ and the solution was washed twice with water $(20 \mathrm{~mL})$. The organic layer was dried over sodium sulfate and the solvent was removed under reduced pressure. The episulfide was cleaned up by means of column chromatography on silica gel with pentane as the eluent.

c) Reduction. The episulfide $(4 \mathrm{mmol})$ was dissolved in anhydrous diethyl ether $(15 \mathrm{~mL})$ and cooled to $0^{\circ} \mathrm{C}$. A solution of lithium aluminium hydride $(0.31 \mathrm{~g} ; 8 \mathrm{mmol})$ 
1159 in anhydrous diethyl ether ( $15 \mathrm{~mL}$ ) was slowly added and then stirred at RT for $2 \mathrm{~h}$. 1160 Anhydrous diethyl ether (30 mL), saturated ammonium chloride solution $(20 \mathrm{~mL})$, and $1161 \mathrm{HCl}(2 \mathrm{~mol} / \mathrm{L} ; 20 \mathrm{~mL})$ were added at $0{ }^{\circ} \mathrm{C}$. The aqueous layer was washed twice with 1162 diethyl ether $(30 \mathrm{~mL})$ and the combined organic layers were washed twice with 1163 saturated sodium hydrogen carbonate solution $(20 \mathrm{~mL})$ and dried over sodium 1164 sulfate. The solvent was evaporated in vacuo and the residue was purified by column 1165 chromatography on silca gel using pentane as the eluent. 
1167 Compound 3

1168

1169

1170

1171

educt: terpinolene

1172

1173

a) $1^{\text {st }}$ intermediate:

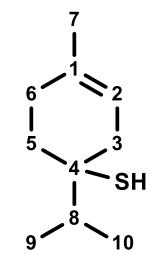

1174

2,2,6-trimethyl-1-oxaspiro[2.5]oct-5-ene,

1175 yield: $89 \%$,

MS-El: m/z (intensity in \%) 79 (100), 110 (62), 95 (35), 77 (20), 91 (16), 93 (16), 94

b) $2^{\text {nd }}$ intermediate:

2,2,6-trimethyl-1-thiaspiro[2.5]oct-5-ene,

1182

yield: $41 \%$,

1183

MS-El: $m / z$ (intensity in \%) 135 (100), 79 (46), 93 (34), 91 (28), 107 (23),

1184

77 (17), 119 (14), 136 (12), 94 (10), 41 (9), 105 (8), 65 (7), 43 (6), 53 (6),

1185

59 (6), 67 (6), 85 (6), $92(6), 75(5), 168\left(5, \mathrm{M}^{+}\right)$

1186

1187

c) analyte:

1188

yield: $23 \%$

1189

1190

RI (FFAP): 1535

RI (DB-5): 1260

MS-El: m/z (intensity in \%) 93 (100), 81 (41), 92 (40), 69 (33), 136 (32), 91 (22), 77 (19), 102 (15), 79 (14), 41 (13), 67 (11), 95 (11), 170 (11, M+), 121 (9), 137 (9), 94

MS-Cl (isobutane): $m / z$ (intensity in \%) $137(100), 171\left(11,[\mathrm{M}+\mathrm{H}]^{+}\right), 138(10), 136(5)$

${ }^{1} \mathrm{H}$ NMR $\left(400 \mathrm{MHz} ; \mathrm{CDCl}_{3}\right): \delta 0.99$ (d, J=1.8 Hz, 3H, H-C10), 1.01 (d, J = $1.8 \mathrm{~Hz}$,

$3 \mathrm{H}, \mathrm{H}-\mathrm{C} 9), 1.39$ (s, SH), $1.54-1.62$ (m, 1H, H-C6), $1.67-1.74$ (m, 1H, H-C8), 1.69 $3 \mathrm{H}, \mathrm{H}-\mathrm{C} 3, \mathrm{H}-\mathrm{C} 5), 5.27-5.31(\mathrm{~m}, 1 \mathrm{H}, \mathrm{H}-\mathrm{C} 2)$ 


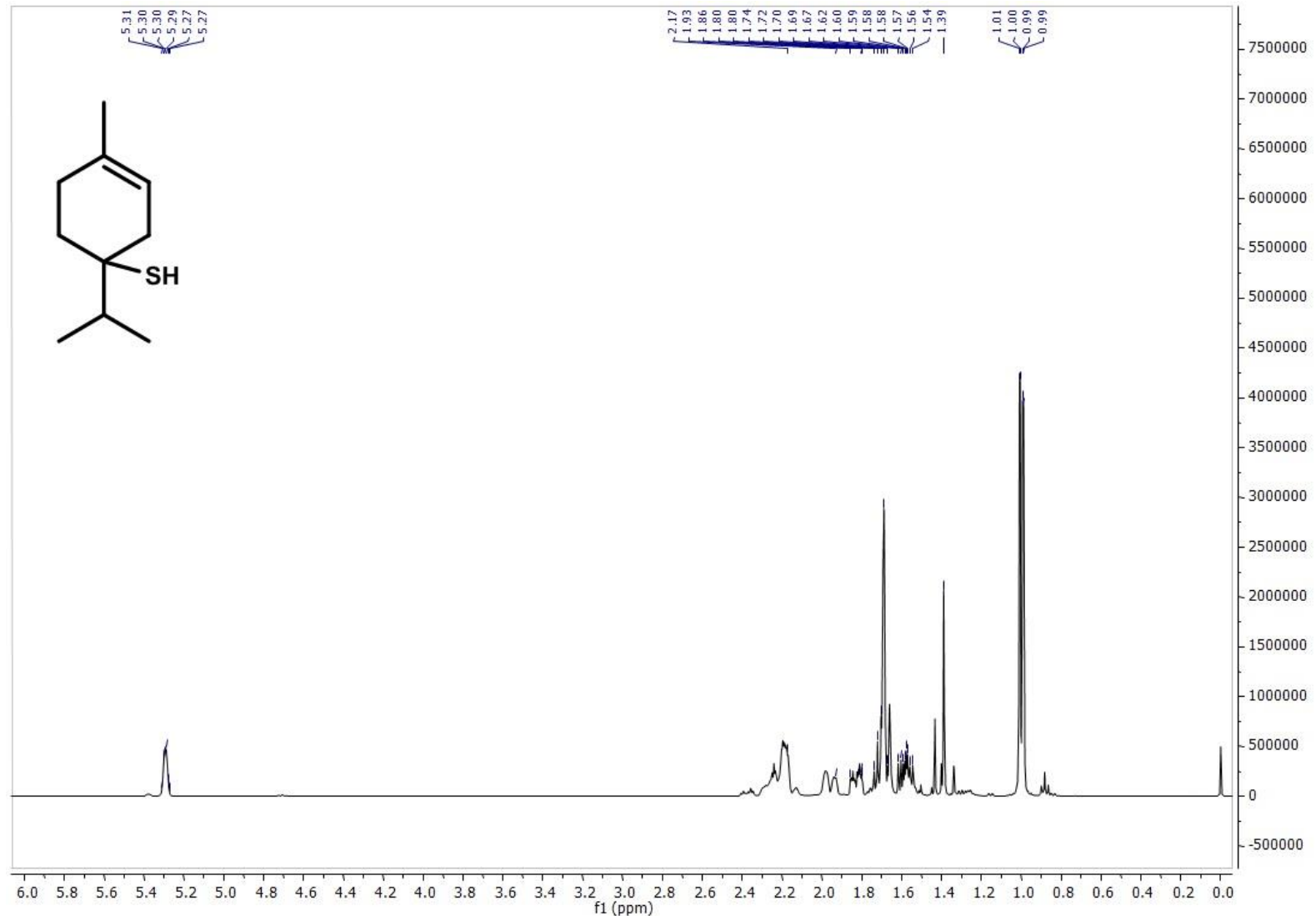

1203 Figure S56. ${ }^{1} \mathrm{H}$ NMR spectrum of 1-p-menthene-4-thiol.

1204

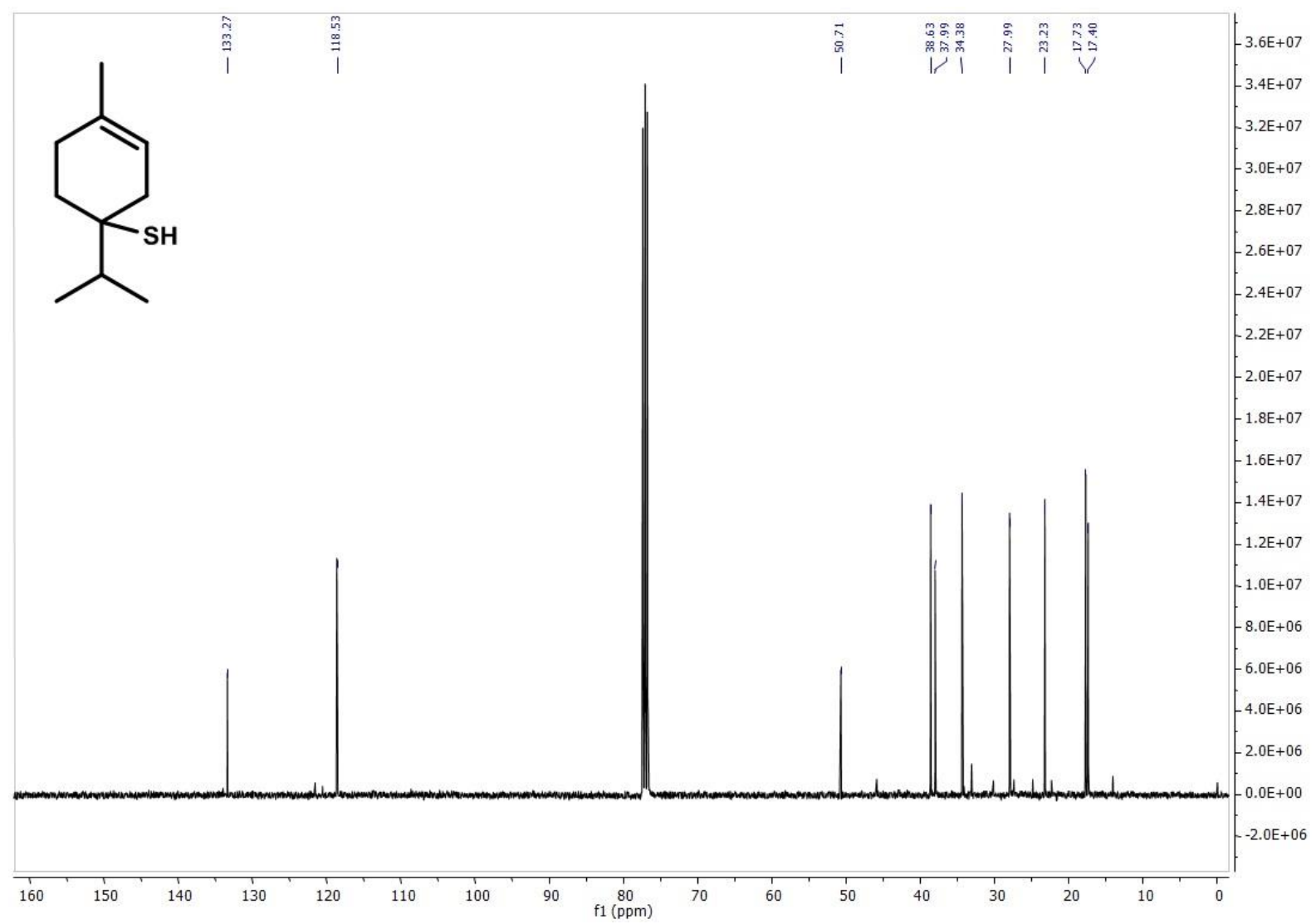

Figure S57. ${ }^{13} \mathrm{C}$ NMR spectrum of 1 -p-menthene-4-thiol. 
1207

1208

1209

1210

1211

1212

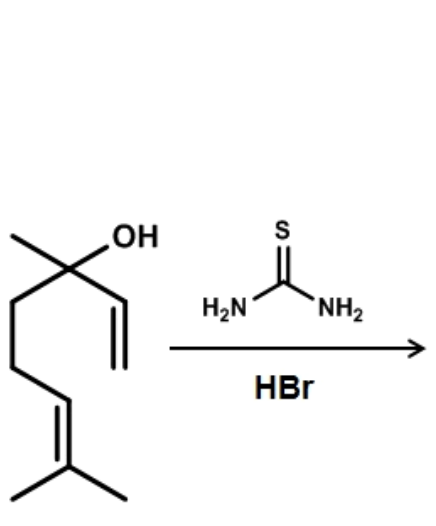

1213

1214 1215

1216 1217 1218 1219 1220 1221 1222 1223 1224 1225 1226 1227 1228 Figure S58.

\section{Synthesis of thio geraniol (compound 19) and thio nerol (21)}

A mixture of thio geraniol and thio nerol was synthesized from linalool as shown in

Figure S58. Synthetic route used for the preparation of thio geraniol and thio nerol.

Linalool (1.54 g; $10 \mathrm{mmol})$ was placed in a round bottom flask and thi ourea $(0.84 \mathrm{~g}$; $11 \mathrm{mmol})$ and hydrogen bromide solution (48\%; $1.5 \mathrm{~mL})$ were added. The solution was stirred at RT for $24 \mathrm{~h}$ and p-toluene sulfonic acid $(2.06 \mathrm{~g} ; 12 \mathrm{mmol})$ in water $(10 \mathrm{~mL})$ was added. After $10 \mathrm{~min}$ the precipitate was filtered off and diethyl ether $(30 \mathrm{~mL})$ was added. The solution was washed twice with water $(10 \mathrm{~mL})$, dried over sodium sulfate and the solvent was removed under reduced pressure. The obtained powder was added to a sodium hydroxide solution $(20 \% ; 15 \mathrm{~mL})$ and stirred at RT for $2 \mathrm{~h}$. Diethyl ether $(20 \mathrm{~mL})$ was added and the organic layer was washed for three times with brine $(15 \mathrm{~mL})$. The aqueous layer was extracted twice with diethyl ether $(20 \mathrm{~mL})$ and the combined organic layers were dried over sodium sulfate. After evaporation of the solvent the compounds were purified by means of column chromatorgraphy on silica gel with pentane as the eluent. 
1229 Compound 19

1230

1231

thio geraniol, yield: $41 \%$

1232

1233

educt: linalool

1234

1235

RI (FFAP): 1599

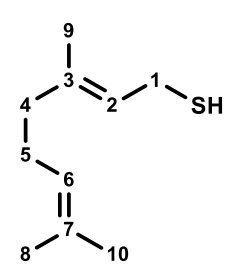

1236

RI (DB-5): 1287

1237

1238

MS-El: $m / z$ (intensity in \%) 41 (51), 155 (18), 67 (16), 93 (12), 123 (9), 53 (8), 59 (8),

1239

101 (8), 137 (6), 81 (5), $\mathrm{M}^{+}$absent

1240

MS-Cl (isobutane): m/z (intensity in \%) 137 (100), 95 (14), 138 (11), 169 (6), 171 (5,

1241

1242 $\left.[\mathrm{M}+\mathrm{H}]^{+}\right)$

1243

1244

1245

${ }^{1} \mathrm{H}$ NMR (400 MHz; $\mathrm{CDCl}_{3}$ ): $\delta 1.39$ (t, J = 7.1 Hz, SH), 1.60 (s, 3H, H-C10), 1.65 (s, $3 \mathrm{H}, \mathrm{H}-\mathrm{C} 9), 1.68$ (s, 3H, H-C8), 1.98 - 2.12 (m, 4H, H-C4, H-C5), $3.14-3.18$ (m, 2H, $\mathrm{H}-\mathrm{C} 1), 5.06-5.10(\mathrm{~m}, 1 \mathrm{H}, \mathrm{H}-\mathrm{C} 6), 5.32-5.37(\mathrm{~m}, 1 \mathrm{H}, \mathrm{H}-\mathrm{C} 2)$

${ }^{13} \mathrm{C}$ NMR (101 MHz, $\mathrm{CDCl}_{3}$ ): $\delta 15.77$ (C9), 17.70 (C10), 22.11 (C1), 25.68 (C8),

1246

26.40 (C5), 39.40 (C4), 123.31 (C2), 123.91 (C6), 131.68 (C7), 137.50 (C3) 
1247 Compound 21

1248

1249 thio nerol, yield: $14 \%$

1250

1251 educt: linalool

1252

1253 RI (FFAP): 1573

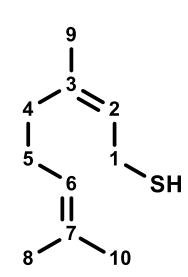

1254 RI (DB-5): 1271

1255

1256

MS-El: $m / z$ (intensity in \%) 69 (100), 41 (55), 93 (25), 67 (20), 137 (16), 68 (14), 155

1257

(14), 53 (13), 81 (10), 59 (9), 101 (8), 91 (7), 121 (7), 79 (6), 89 (6), 123 (5), 136 (5),

1258 $170\left(3, \mathrm{M}^{+}\right)$

1259

MS-Cl (isobutane): $m / z$ (intensity in \%) 137 (100), 95 (19), $171\left(16,[\mathrm{M}+\mathrm{H}]^{+}\right), 91(14)$,

1260 93 (11), 138 (11), 109 (5)

1261

${ }^{1} \mathrm{H}$ NMR (400 MHz; $\mathrm{CDCl}_{3}$ ): $\delta 1.40$ (s, J = 7.1 Hz, SH), 1.62 (s, 3H, H-C10), 1.68 (s,

1262 $3 \mathrm{H}, \mathrm{H}-\mathrm{C} 8$ ), 1.69 (s, 3H, H-C9), 1.98 - 2.12 (m, 4H, H-C4, H-C5), $3.12-3.16$ (m, 2H, 1263 $\mathrm{H}-\mathrm{C} 1), 5.09-5.12(\mathrm{~m}, 1 \mathrm{H}, \mathrm{H}-\mathrm{C} 6), 5.32-5.37$ (m, 1H, H-C2)

1264

${ }^{13} \mathrm{C}$ NMR (101 MHz, $\mathrm{CDCl}_{3}$ ): $\delta 17.68$ (C9), 22.00 (C10), 23.25 (C1), 25.71 (C8), 1265 26.49 (C5), 31.68 (C4), 123.88 (C2), 124.17 (C6), 132.09 (C7), 137.42 (C3) 


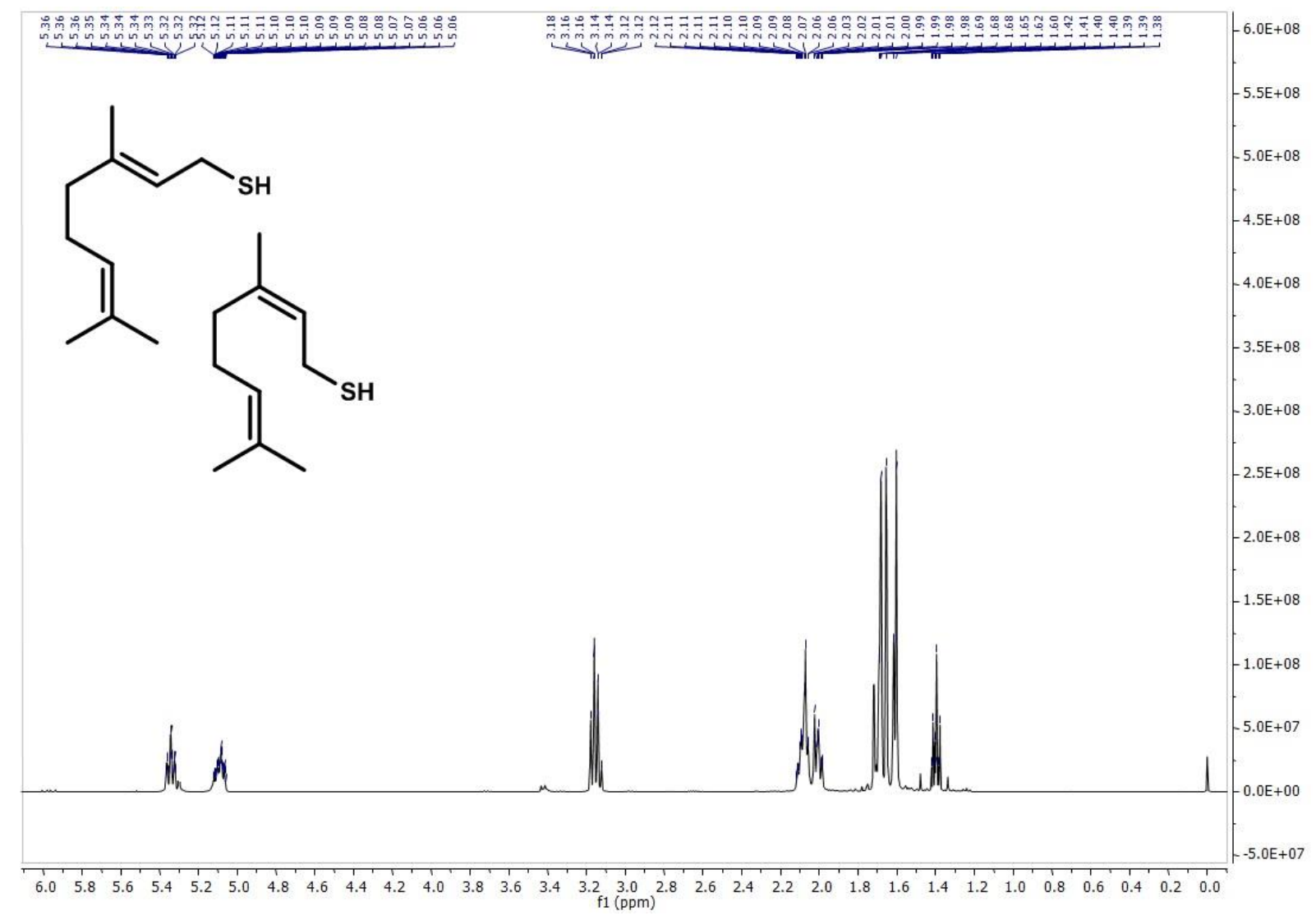

Figure S59. ${ }^{1} \mathrm{H}$ NMR spectrum of a mixture of thio geraniol and thio nerol.

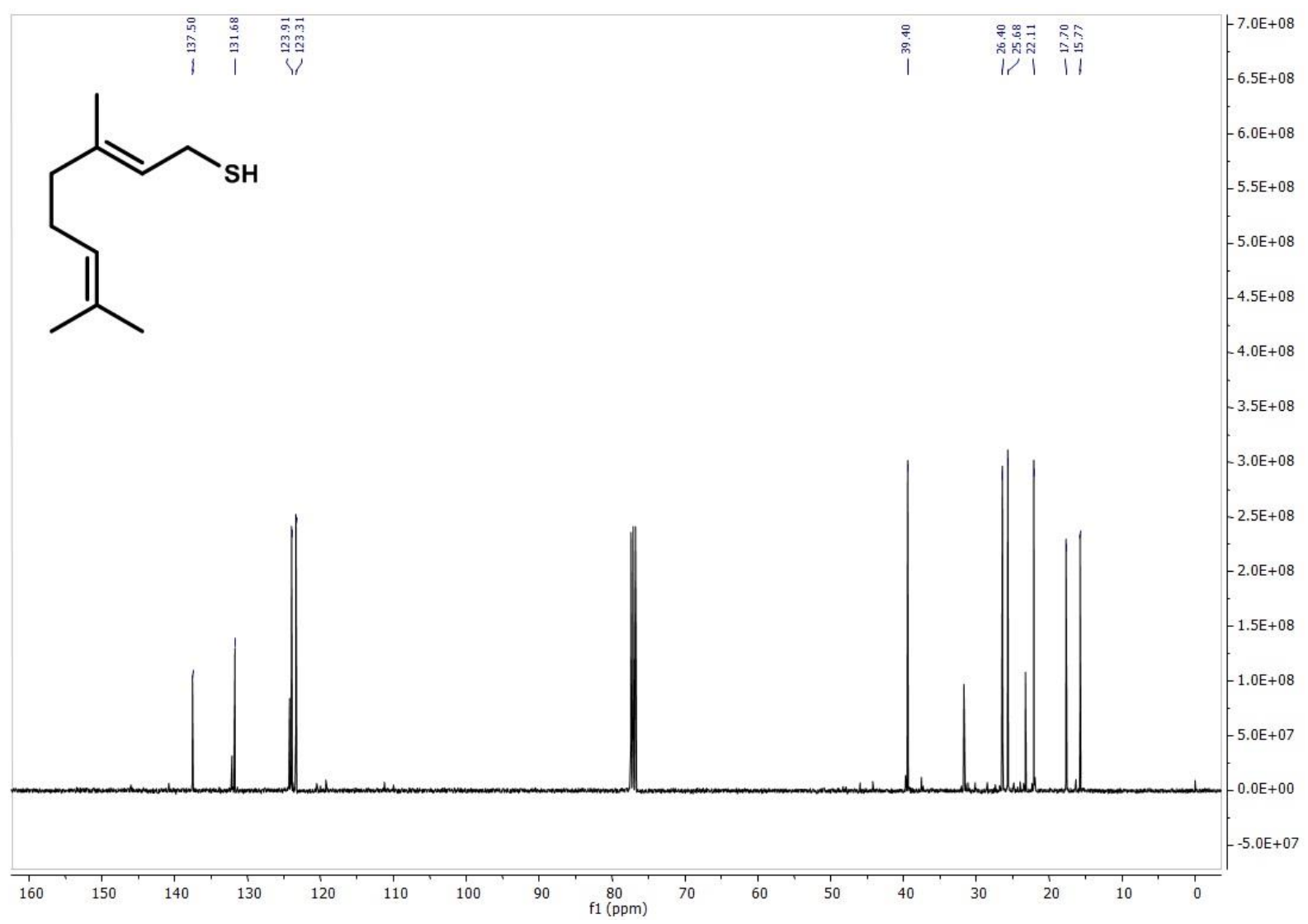

Figure S60. ${ }^{13} \mathrm{C}$ NMR spectrum of a mixture of thio geraniol and thio nerol; signals of 1270 thio geraniol. 


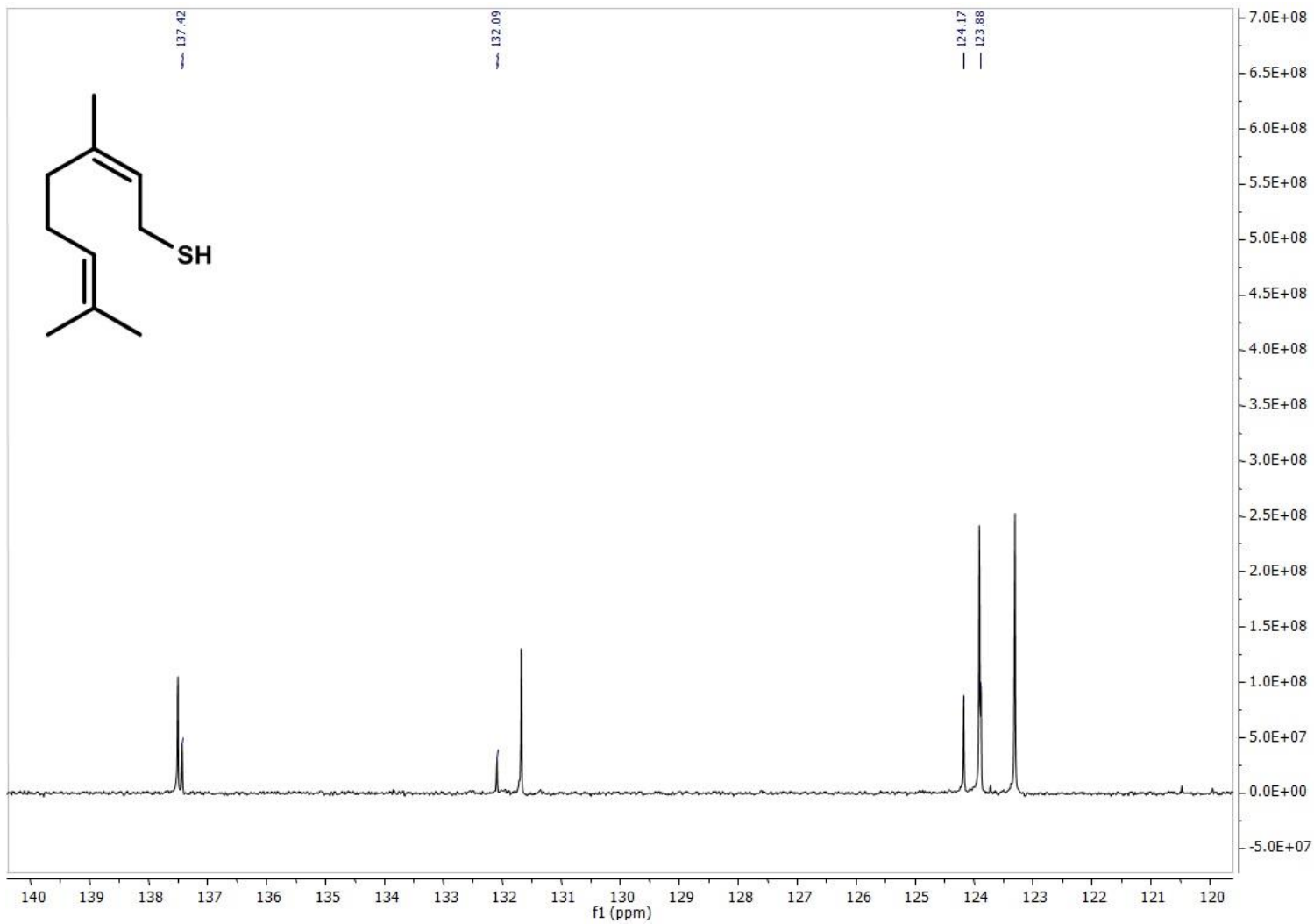

1272 Figures S61. ${ }^{13} \mathrm{C}$ NMR spectrum of a mixture of thio geraniol and thio nerol; signal of 1273 thio nerol I.

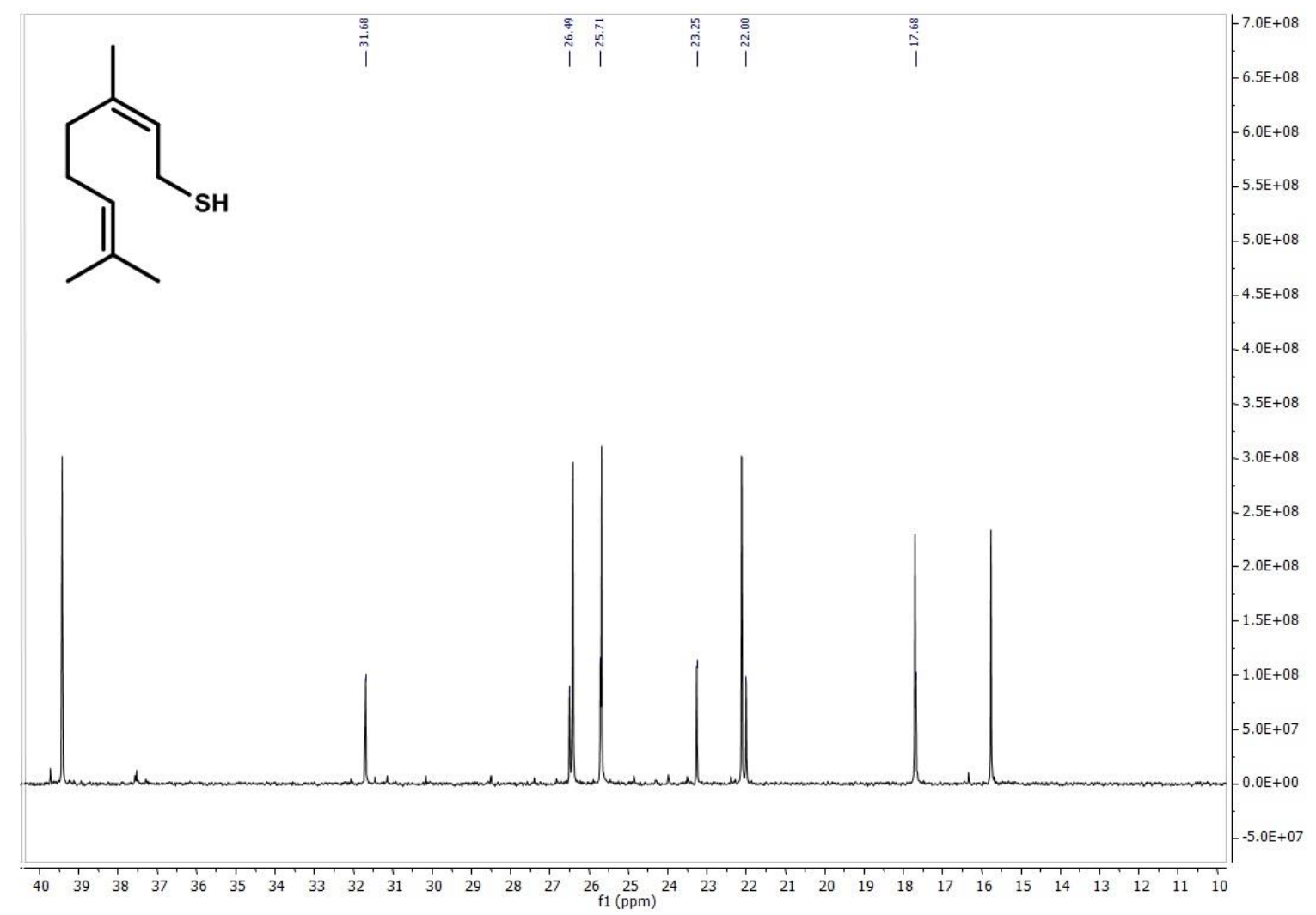

Figures S62. ${ }^{13} \mathrm{C}$ NMR spectrum of a mixture of thio geraniol and thio nerol; signals 1276 of thio nerol II. 
1277 Synthesis of thio linalool (20)

1278

1279

Synthesis of thio linalool was done from geraniol, as shown in Figure $\mathbf{S 6 3 .}$

1280

1281<smiles>CC(C)=CCCC(C)=CCO</smiles>

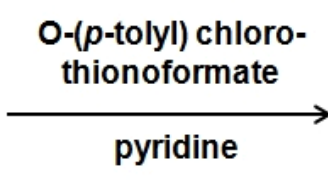

1282

1283

1284

1285

1286

1287

1288

1289

1290

1291

1292

Figure S63. Synthetic route used for the preparation of thio linalool.

Geraniol $(1.54 \mathrm{~g} ; 10 \mathrm{mmol})$ was dissolved in pyridine $(15 \mathrm{~mL})$ and cooled to $0{ }^{\circ} \mathrm{C}$. $\mathrm{O}$ ( $p$-tolyl) chloro thionoformate $(1.87 \mathrm{~g} ; 10 \mathrm{mmol}$ ) was carefully added and the solution was stirred at RT for $2 \mathrm{~h}$. Diethyl ether $(30 \mathrm{~mL})$ was added and the reaction mixture was washed twice with $2 \mathrm{~N}$ sodium hydroxide solution $(20 \mathrm{~mL}), 2 \mathrm{~N}$ hydrogen chloride solution $(20 \mathrm{~mL})$ and brine $(20 \mathrm{~mL})$. The solvent was removed under reduced pressure. The intermediate was reduced with lithium aluminium hydride and purified by column chromatography on silica gel with pentane as the eluent. 
1293 Compound 20

1294

1295

thio linalool, yield: $14 \%$

1296

1297

educt: geraniol

1298

1299

RI (FFAP): 1439

1300

RI (DB-5): 1194

1301

1302

MS-El: m/z (intensity in \%) 41 (100), 69 (95), 93 (77), 55 (38), 67 (33), 53 (30), 80

1303

(30), 81 (29), 43 (26), 45 (22), 121 (19), 79 (18), $136\left(18, \mathrm{M}^{+}\right), 87$ (16), 68 (15), 44

1304

(14), 91 (14), 92 (14), 95 (14), 59 (12), 94 (8), 107 (8), 127 (6)

1305

MS-Cl (isobutane): $m / z$ (intensity in \%) $137(100), 91(45), 95(43), 171\left(11,[\mathrm{M}+\mathrm{H}]^{+}\right.$),

1306

107 (9), $136(9), 138(7), 123(6), 172(5)$

1307

${ }^{1} \mathrm{H}$ NMR (400 MHz; $\mathrm{CDCl}_{3}$ ): $\delta 1.48$ (s, 3H, H-C9), 1.60 (s, 3H, H-C10), 1.65 - 1.69

1308

(m, 2H, H-C4), 1.68 (s, 3H, H-C8), 1.78 (s, SH), 1.98 - 2.09 (m, 2H, H-C5), 4.96 -

1309

4.99 (m, 1H, H-C1), $5.06-5.12$ (m, 2H, H-C1, H-C6), 5.97 (dd, J = 17.2 Hz, 10.6 Hz,

1310

$1 \mathrm{H}, \mathrm{H}-\mathrm{C} 2)$

1311

${ }^{13} \mathrm{C}$ NMR (101 MHz, $\mathrm{CDCl}_{3}$ ): $\delta 17.64$ (C10), 23.98 (C5), 25.66 (C8), 28.51 (C9),

1312

44.20 (C4), 48.29 (C3), 111.15 (C1), 123.72 (C6), 131.94 (C7), 145.95 (C2) 


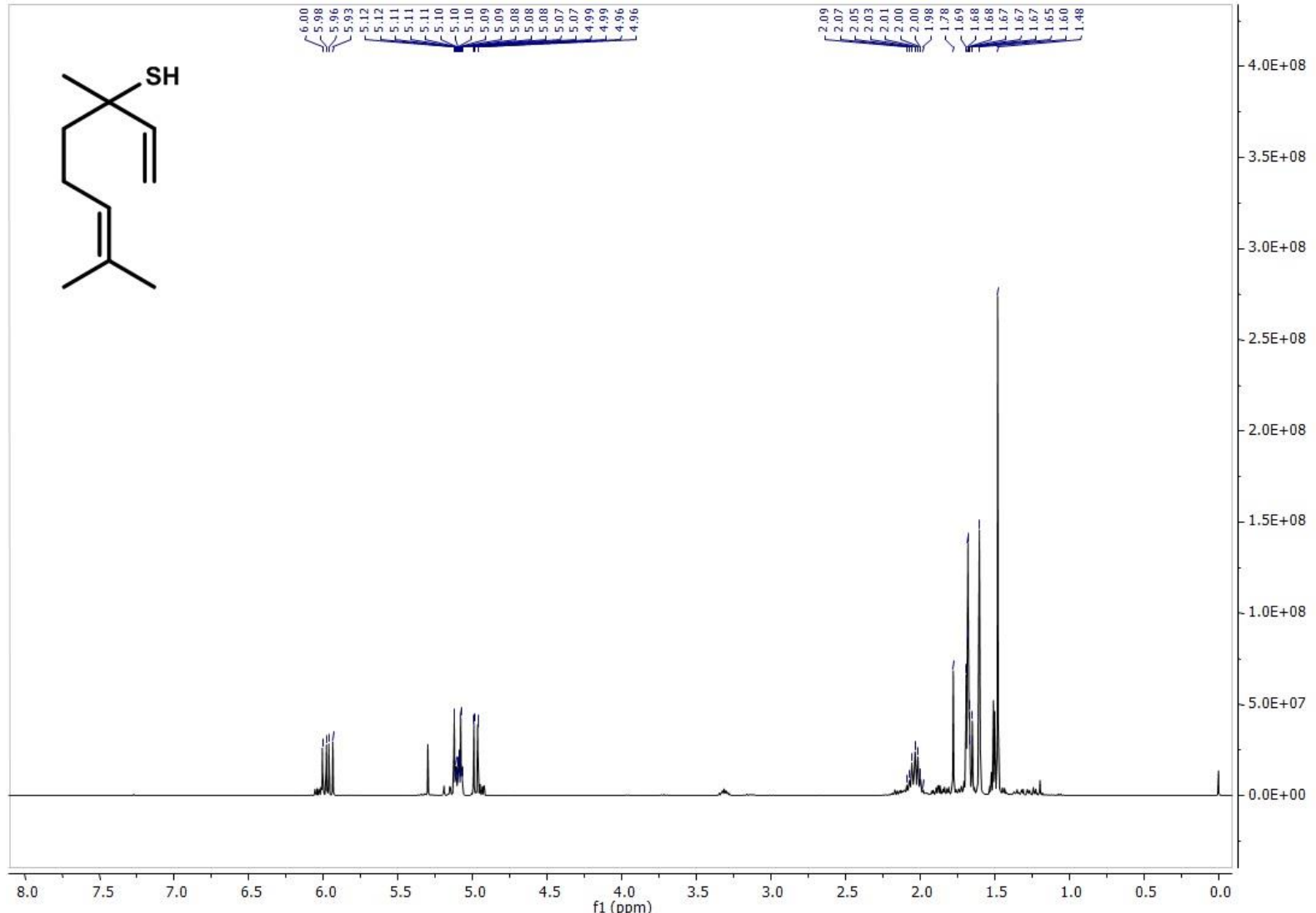

1314 Figure S64. ${ }^{1} \mathrm{H}$ NMR spectrum of thio linalool.

1315

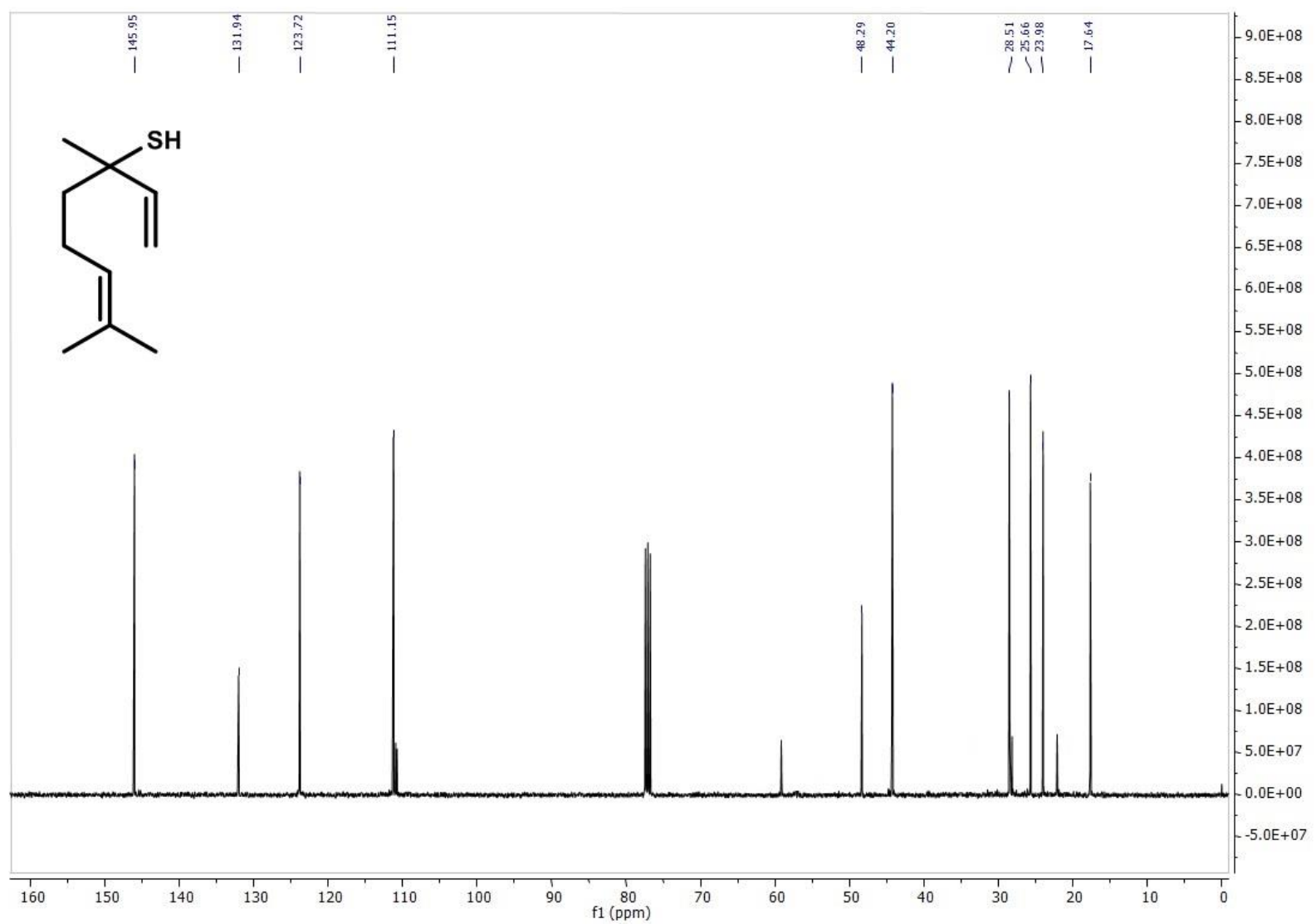

1317 Figure S65. ${ }^{13} \mathrm{C}$ NMR spectrum of thio linalool. 


\section{Further spectroscopic data}

1319

1320 Compound 14

1321

1322

p-cymene-3-thiol

1323

1324

RI (FFAP): 1867

RI (DB-5): 1315

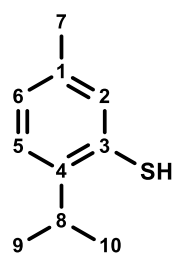

1326

1327

MS-El: $m / z$ (intensity in \%) 151 (100), $166\left(44, \mathrm{M}^{+}\right), 91$ (33), 149 (31),

1328

115 (28), 136 (22), 162 (21), 117 (20), 161 (20), 118 (15), 39 (14), 45

1329

(14), 65 (14), 77 (14), 105 (14), 124 (14), 134 (14), 147 (14), 41 (12), 116 (9), 119 (9),

1330

51 (8), 59 (8), 152 (7), 89 (5), 92 (5), 167 (5)

1331

MS-Cl (isobutane): $m / z$ (intensity in \%) $167\left(100,[\mathrm{M}+\mathrm{H}]^{+}\right), 166(16), 168(15), 125$

1332

(9), $165(7)$

1333

${ }^{1} \mathrm{H}$ NMR (400 MHz; $\left.\mathrm{CDCl}_{3}\right): \delta 1.23$ (d, J = 6.8 Hz, 6H, H-C9, H-C10), 2.26 (s, 3H, H-

1334

C7), $3.14-3.21$ (m, 1H, H-C8), 3.24 (s, SH), 6.96 (d, J=7.9 Hz, 1H, H-C6), 7.09 (s,

1335

$1 \mathrm{H}, \mathrm{H}-\mathrm{C} 2), 7.12$ (d, J = $7.9 \mathrm{~Hz}, 1 \mathrm{H}, \mathrm{H}-\mathrm{C} 5)$

1336

${ }^{13} \mathrm{C}$ NMR (101 MHz, CDCl $)$ : $\delta 20.70$ (C7), 22.91 (C9, C10), 30.93 (C8), 125.52 (C5),

1337

127.16 (C6), 129.39 (C3), 131.27 (C2), 135.89 (C1), 143.43 (C4) 


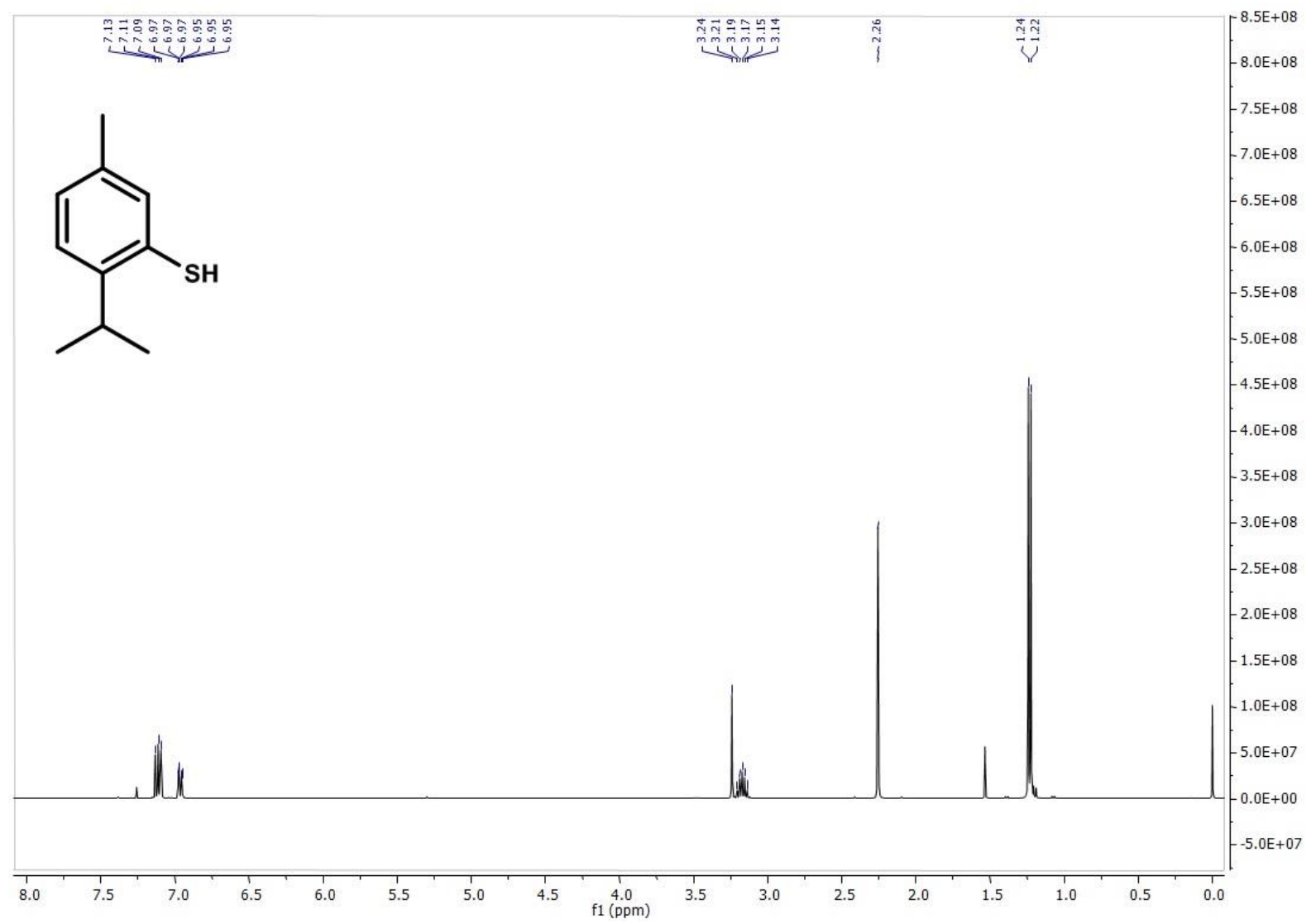

Figure S66. ${ }^{1} \mathrm{H}$ NMR spectrum of $p$-cymene-3-thiol.

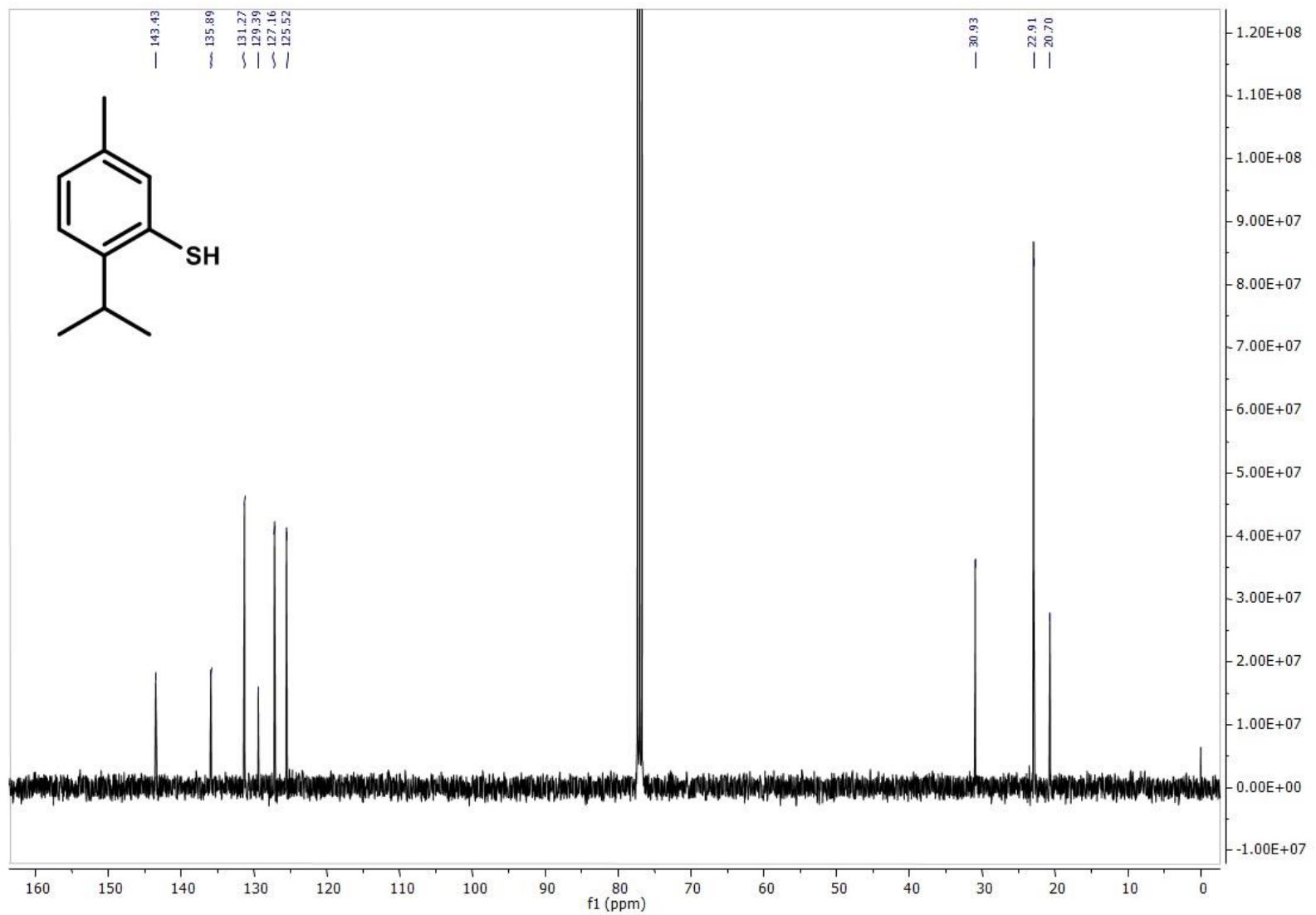

Figure S67. ${ }^{13} \mathrm{C}$ NMR spectrum of $p$-cymene-3-thiol. 